\title{
Теория Стационарной Самосогласованной Вселенной
}

\section{Александр Авшалумов,}

Head of Moscow Research Institute for Cybernetic Medicine (MRICM), Miklouho-Maclay str. 16/10, building 90, 117437, Moscow, Russia

\section{Абстракт}

С момента создания ОТО и последующих работ в космологии, вопрос о кривизне пространства во Вселенной $(\omega>0, \omega<0$ или $\Omega=0)$ - считается один из наиболее главных и дискутируемых по сей день. Это естественно, ведь от кривизны пространства зависит: расширяется Вселенная, сжимается или является статичной. Эти дискуссии, позволили автору выдвинуть парадоксальную идею: одновременного существования во Вселенной трех взаимосвязанных пространств-времен (положительной, отрицательной и нулевой кривизны) и, уже на этой основе, разработать теорию, в которой каждое пространство-время играет свою собственную роль и развивается в строгом соответствии, со своим знаком кривизны.

В предлагаемой работе исследуется, гипотеза о новой пространственно-временной симметрии во Вселенной, согласно которой в первичном (материнском) обобщенном Абсолютном пространстве-времени ${ }^{A} S T$ нулевой $(\Omega=0)$ кривизны с геометрией Евклида $(\mathcal{E})$ при взаимодействии со сферой с расстоянием-радиусом и кривизной, равными мнимой параболической единице $I\left(S_{s p h}=I, \Omega_{s p h}=I\right)$, в момент Большого Взрыва $(B B)$ синхронно родились два взаимопрозрачных некоммутативных самосогласованных пространства-времени:

- горячее пространство - время ${ }^{L} S T$ постоянной положительной $\left({ }^{L} \omega>0\right)$ кривизны с положительной плотностью материи и энергии, в которой реализуется геометрия Римана $(\mathcal{R})$ эллиптическая, ткань пространства однородно расширяется (относительно тканей Абсолютного пространствавремени $\left.{ }^{A} S T\right)$ во времени ${ }^{L} T$;

- холодное пространство - время ${ }^{D} S T$ постоянной отрицательной $\left({ }^{D} \omega<0\right)$ кривизны с отрицательной плотностью материи и энергии, в которой реализуется геометрия Лобачевского-Больяи $(\mathcal{L B})$ гиперболическая, ткань пространства неоднородно сжимается (относительно тканей Абсолютного пространства-времени ${ }^{A} S T$ ) во времени ${ }^{D} T$.

В любой момент времени синхронизации $\left(T_{\text {synch }}\right)$ выполняется следующее уравнение $\left[{ }^{L} \omega\left(T_{\text {synch }}\right) \cdot{ }^{D} \omega\left(T_{\text {synch }}\right)=I\right]$, где $I=|\mathbf{1}|$.

Данная космологическая модель в корне решает проблему наблюдаемой барионной асимметрии и скрытой массы во Вселенной, поскольку с момента $B B$ вся рожденная материя развивается, в наблюдаемом нами, пространстве-времени положительной кривизны ${ }^{L} S T$, а вся рожденная антиматерия развивается, в не наблюдаемом нами, пространстве-времени отрицательной кривизны ${ }^{D} S T$.

Такой подход позволяет распознать трех пространственно-временную природу спина, разработать его механизм, который напрямую приводит к новой трех пространственно-временной самосогласованной частице-солитонной модели атома и атомного ядра, что позволяет дать новую интерпретацию квантовой механике. 


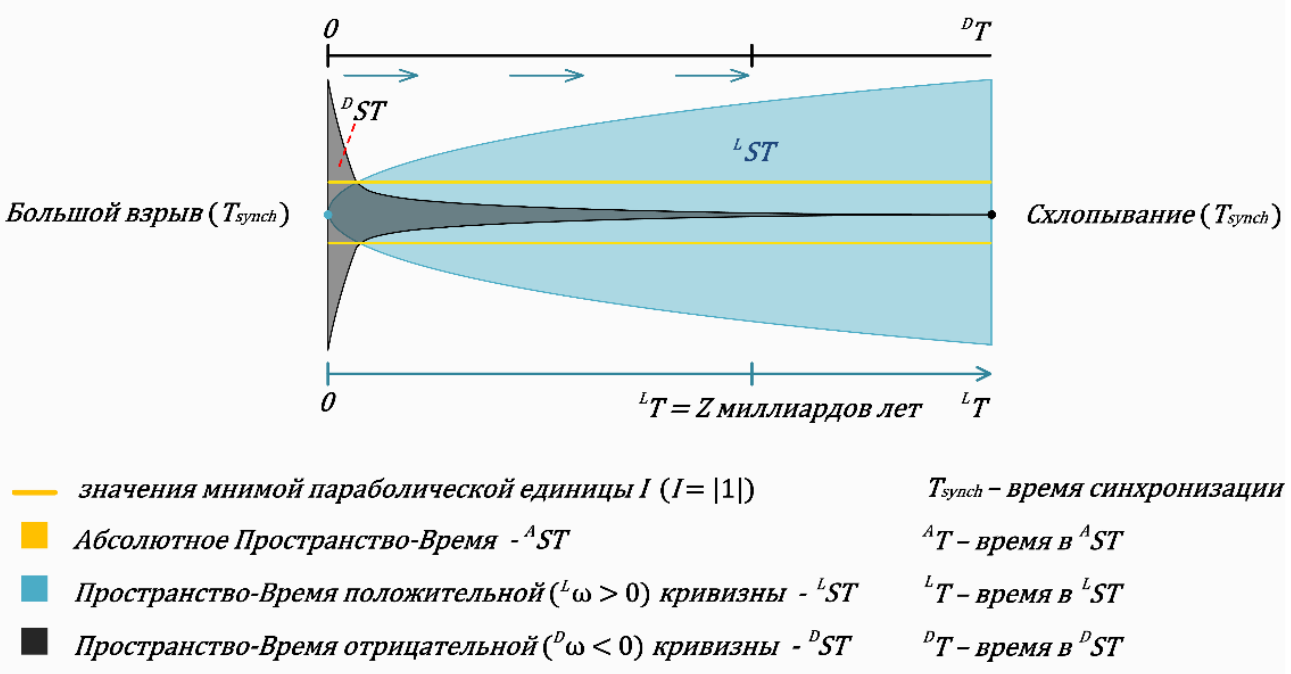

Графический абстракт: трех пространственно-временная модель строения Вселенной.

По мнению автора, трех пространственно-временная модель строения Вселенной позволяет решить многие вопросы современной космологии и теоретической физики, создает основу для построения единой физической теории (в том числе, объединяющей ОТО и квантовую физику) и дает надежду в обозримом будущем, разработать количественную логически-завершенную модель рождения $B B$ и последующего схлопывания $\mathrm{Cr}$ двух взаимопрозрачных некоммутативных самосогласованных пространств-времен постоянной положительной и постоянной отрицательной кривизны. 


\section{Преамбула}

«... может быть, именно то, что мы называем мнимым временем, на самом деле более фундаментально, а то, что мы называем временем реальным - это некое субъективное представление, возникшее у нас при попытках описать, какой мы видим Вселенную».

Stephen Hawking «A Brief History of Time», 1988.

«Квантовая механика, действительно, впечатляет. Но внутренний голос говорит мне, что это еще не идеал. Эта теория говорит о многом, но все же не приближает нас к разгадке тайны Всевышнего. По крайней мере, я уверен, что Он не бросает кости».

Albert Einstein «Letter to Max Born», December 4, 1926

«... Бог - совершенный математик и Он использовал самую продвинутую математику при создании Вселенной».

Paul Adrien Maurice Dirac «The Evolution of the Physicist's Picture of Nature», May issue Scientific American, 1963 


\section{Содержание}

Абстракт

Глава 1 Вступление .7

Глава 2 Исходные положения TSSCU. .10

2.1 Понятие времени синхронизации $-T_{\text {synch }}$ 12

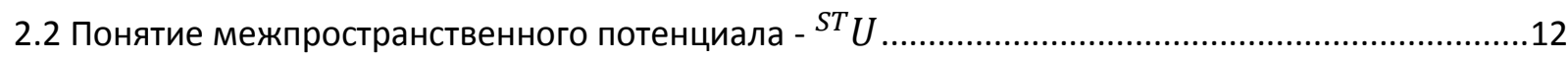

2.3 Понятие фундаментальной сцепленности - æ. 12

Глава 3 Первичное (материнское) Абсолютное пространство-время ${ }^{A} S T$ как основа для модели Большого Взрыва $(B B)$ и последующего Схлопывания $(C r)$

3.1 Цель и условия задачи .13

3.2 Базовые данные .13

3.3 Первое стартовое уравнение 13

3.4 Алгоритм 1. Умножение на мнимую единицу $[i]$ .14

3.5 Алгоритм 2. Возведение в степень и умножение на отрицательную единицу [-1] 15

3.6 Гравитация и температура. Основное стартовое уравнение .19

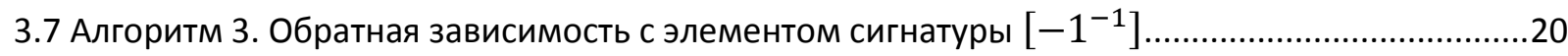

3.8 Алгоритмы и частичное расслоение Абсолютного пространства-времени ${ }^{A} S T$......................24

3.9 Алгоритмы и Управляющая Супермнимая Единица ${ }_{S} I$......................................................24

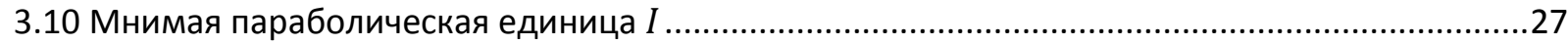

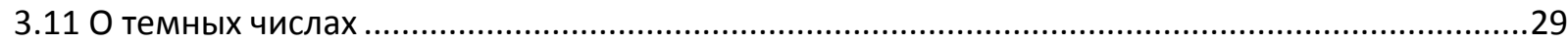

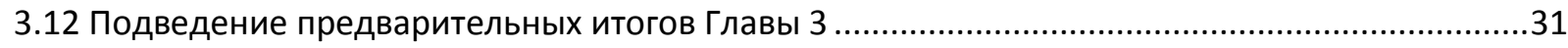

Глава 4 Свойства и характеристики первичного (материнского) Абсолютного пространствавремени ${ }^{A} S T$

4.1 Введение 32

4.2 Соответствие каждого 0 и $\infty$. Неразрывность этих понятий и значений друг от друга в пространства-времени ${ }^{A} S T$

4.3 Изменение свойств и характеристик пространства-времени ${ }^{A} S T$ в зависимости от периода Релаксации или периода от Большого Взрыва до очередного Схлопывания ...............................33

4.4 Период Релаксации $\left(C r_{n-1}-B B_{n}\right)$. Состояние неопределенности базовых параметров ........33

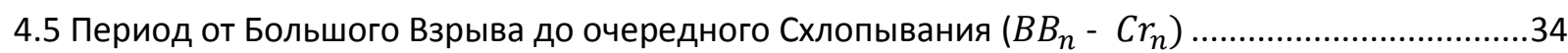

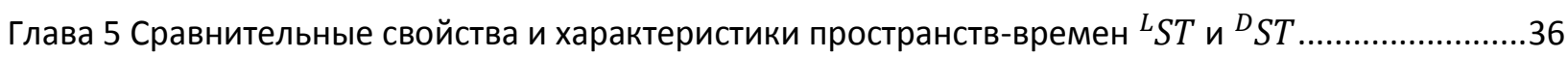

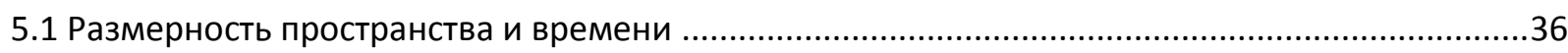

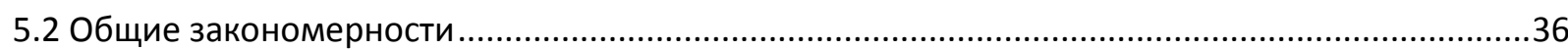

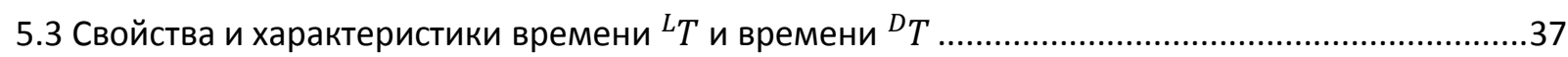

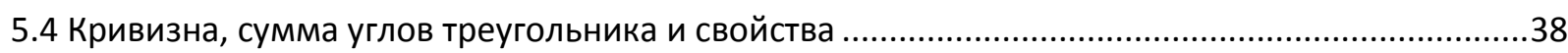

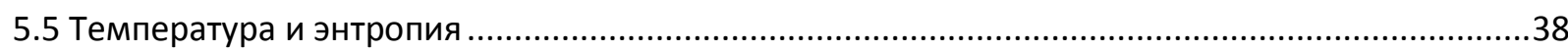

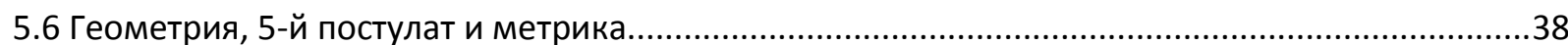


5.7 Материя, энергия и информация

5.8 Элементарный электрический заряд $q$ и элементарный топологический магнитный заряд $\tilde{\theta}$

5.9 Электромагнитные волны (ЭМВ) и позитронно-электрические волны (ПЭВ) ........................40

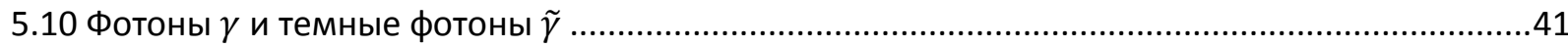

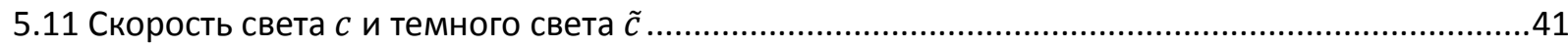

Глава 6 Трех пространственно-временная модель квантовой положительной гравитации в ${ }^{L} S T$ и

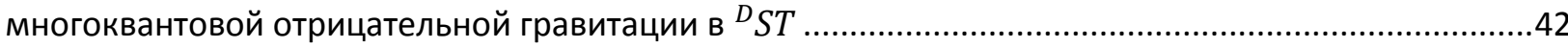

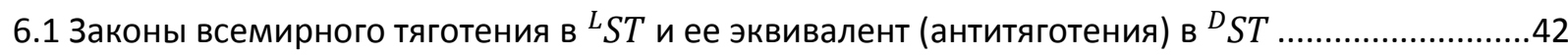

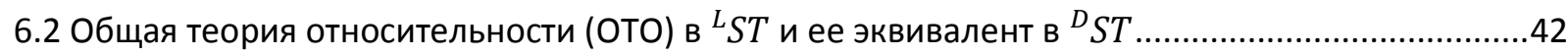

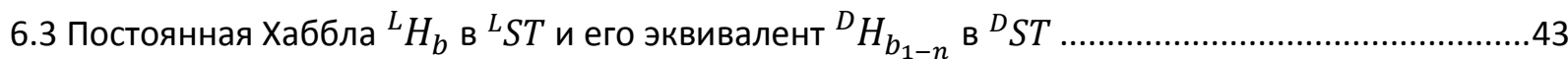

6.4 Ткани пространств-времен ${ }^{A} S T,{ }^{L} S T$ и ${ }^{D} S T$ - как реальные и объективные составляющие устройства Вселенной

6.5 Образование масс ${ }^{L} m$ частиц материи в ${ }^{L} S T$ и масс ${ }^{D} m$ децентрализованных объектов антиматерии в ${ }^{D} S T$.

6.6 Самосогласованные уравнения рождения и эволюции тканей пространств-времен в условиях гравитации в ${ }^{L} S T$ и антигравитации в ${ }^{D} S T$

6.7 Классификация и новый содержательный смысл петлевой квантовой гравитации $L Q G$ и теории струн $S T$ на базе теории взаимопрозрачных самосогласованных сетей в условиях гравитации в ${ }^{L} S T$ и антигравитации в ${ }^{D} S T$.

6.8 Форма материи в ${ }^{L} S T$ в условиях гравитации и форма антиматерии в ${ }^{D} S T$ в условиях

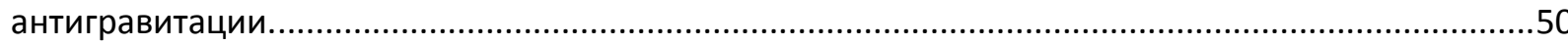

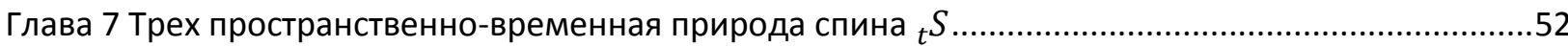

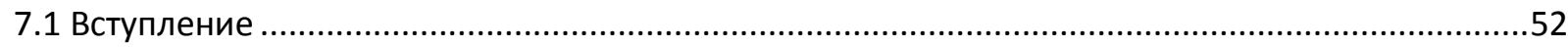

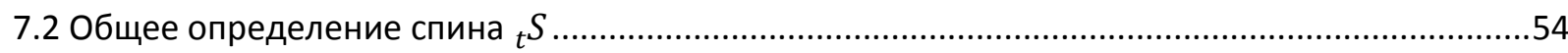

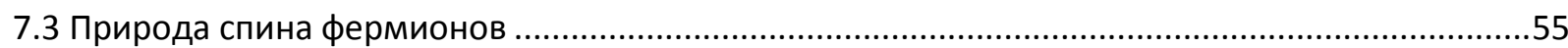

7.3.1 Общие положения и определение ........................................................................55

7.3.2 Геометрическое расположение спина фундаментально-сцепленных фермионов в

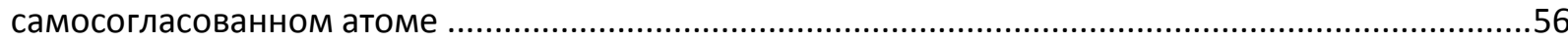

7.3.3 Геометрическое расположение спина при движении свободных (вне атома или ядра) фундаментально-сцепленных фермионов по осциллирующим незамкнутым орбитам ...............58

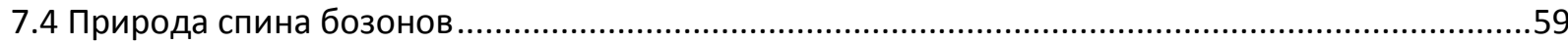

7.4.1 Общие положения и определение .........................................................................59

7.4.2 Геометрическое расположение спина при движении фундаментально-сцепленных безмассовых бозонов по осциллирующим незамкнутым орбитам

7.4.3 Особенности спина при движении фундаментально-сцепленных массивных векторных $\pm W$ бозонов

7.4.4 Особенности спина массивного векторного ${ }^{0} Z$ бозона. Предсказание. .63

7.4.5 Спин бозона Хиггса $H$ 
7.5 Заключение .63

Глава 8 Самосогласованная частице-солитонная модель атома водорода (протия) в трех пространствах-времени ${ }^{A} S T,{ }^{L} S T,{ }^{D} S T$. 64

Глава 9 Фундаментальные взаимодействия в пространствах-времени ${ }^{L} S T$ и ${ }^{D} S T \ldots \ldots \ldots \ldots \ldots \ldots \ldots . . . . . . . . .66$

Глава 10 Поля и преобладающие процессы в пространствах-времени ${ }^{L} S T$ и ${ }^{D} S T$...........................68

Глава 11 Принципы движения материальных тел в пространствах-времени ${ }^{L} S T$ и ${ }^{D} S T$.................69

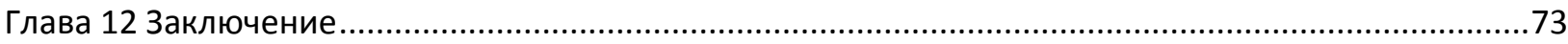

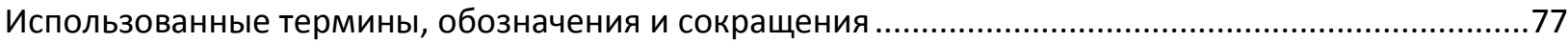

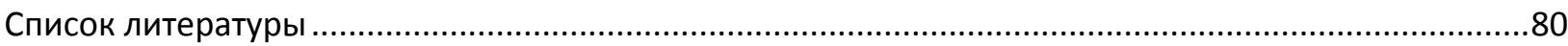

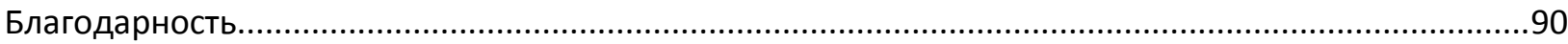




\section{Глава 1 Вступление}

Кривизна пространства во Вселенной, ее размерность, пространственно-временные и геометрические отношения, реализованные в ней - являются главными вопросами космологии и теоретической физики. Общая теория относительности [1, 2], как лучшая теория гравитации - создала основу и придала этим направлениям исследований дополнительный импульс $[3,4,5,6,7,8,9,10,11,12,13,14,15,16,17$, $18,19,20,21,22,23,24,25,26,27,28]$. Стандартная модель сегодня - является общепризнанной и лучшей теорией, описывающей фундаментальные взаимодействия [29]. Однако, в современной физике накопилось немало разнообразных проблем, которые требуют своего разрешения [30, 31, 32]. Помимо вышеуказанных, это прежде всего: наблюдаемая барионная асимметрия во Вселенной [33, 34]. Почему, несмотря на незыблемость второго закона термодинамики, мы не наблюдаем тепловой смерти Вселенной $[35,36]$ ? Неполнота инфляционной модели Большого Взрыва [37, 38, 39, 40, 41, 42, 43, 44, 45, 46, 47]. Куда «подевалась» холодная стадия развития Вселенной $[48,49,50]$ ? Осциллирует ли Вселенная $[51,52,53,54,55,56,57,58]$ ? Непонимание природы темной материи и темной энергии, а также почему наблюдаемое пространство продолжает ускоренно расширяться? Какова природа массы? Какова фундаментальная роль поля БраутаЭнглера-Хиггса и бозона Хиггса? Полна ли теория электрослабого взаимодействия? Непонимание происхождения сил инерции. Где скрывается элементарный магнитный заряд? Что из себя представляют элементарный электрический и элементарный магнитный заряды? Непонимание природы спина бозонов и фермионов. Какова природа ядерных сил и конфаймента? Что из себя представляет механизм туннельного эффекта? Отсутствие полноценной теории сверхпроводимости. Почему существуют три поколения элементарных частиц? Каким образом дать корректное определение понятию физического поля? Что из себя представляют компактные массивные объекты, наблюдаемые в астрономии? Отсутствие теории квантовой гравитации.

Для того, чтобы постараться ответить на эти вопросы требуются принципиально новые идеи, лежащие в самой основе теории $B B$ и позволяющие напрямую связать космологию, как с уже известными, так и еще не открытыми законами физики. Разработанная автором, трех пространственно-временная (положительной, отрицательной и нулевой кривизны) модель строения стационарной самосогласованной Вселенной предоставляет новые идеи и широкие возможности для построения единой физической теории. Данная модель позволяет получить шесть базовых параметров в каждом пространстве-времени, отвечающих за эволюцию Вселенной в период с $B B$ до $C r$ (см. Главу 3), а также делает понятным подход к определению размерности Вселенной (см. Главу 12).

В космологии, естественным образом решается проблема наблюдаемой барионной асимметрии и скрытой массы, поскольку с момента $B B$ вся рожденная материя развивается, в наблюдаемом нами, пространстве-времени положительной кривизны ${ }^{L} S T$, а вся рожденная антиматерия развивается, в не наблюдаемом нами, пространстве-времени отрицательной кривизны ${ }^{D} S T$ (см. Главу 5 ). 
Переход от макроуровня к микрообъектам позволяет распознать трех пространственно-временную природу спина и разработать его механизм (см. Главу 7), который напрямую приводит к трех пространственно-временной самосогласованной частице-солитонной модели атома (см. Главу 8). Такой подход позволяет дать новую интерпретацию квантовой механики, раскрывающей смысл уравнений Шредингера и Дирака (см. Главу 11), а также непротиворечиво и существенно дополнить современную квантовую теорию элементарных частиц.

Выход из «плена неопределенности Гейзенберга» становится возможным, благодаря новому способу введения обобщенных комплексных чисел (см. пп. 3.9, 3.10 Главы 3 и Главу 4). Каждая мнимая единица: параболическая $I$, эллиптическая $i$ и гиперболическая $\tilde{l}$ - задается в строгом соответствии со знаком кривизны своего пространства-времени. Именно, обобщенные комплексные числа, развивающие теории функций комплексных переменных, каждая, строго в своем пространствевремени, играют главную роль в обеспечении межпространственно-временной коммуникации и делают возможным «эффект самосогласованности» во Вселенной.

Автор также был вынужден ввести новые понятия, термины и обозначения, без которых формализация данной теории была бы невозможна. Одним из таких, новых принципиальных понятий - является Управляющая Супермнимая Единица ${ }_{s} I$, которая в период с $C r$ до очередного $B B$ вмещает в себя мнимые: параболическую $I$, эллиптическую $i$ и гиперболическую $\tilde{l}$ единицы и, по мнению автора, наряду с первичным (материнским) Абсолютным пространством-временем ${ }^{A} S T$, играет важнейшую роль во Вселенной (см. Главу 4).

Исследование возможностей построения самосогласованной модели трех пространственно-временной квантовой положительной гравитации в ${ }^{L} S T$ и многоквантовой отрицательной гравитации в ${ }^{D} S T$, рассматривается в Главе 6. Вопервых, с учетом того, что одним из шести базовых параметров - является гравитация, удается получить относительно простые самосогласованные формулы для определения гравитации и антигравитации (см. п. 6.6). Во-вторых, новое понимание и определения получают сами понятия: положительной гравитации и отрицательной гравитации (антигравитации). В-третьих, новый содержательный смысл и импульс получают, две взаимодополняющие друг друга, теории: петлевой квантовой гравитации (далее - $L Q G$ ) и теории струн (далее - ST) на базе теории взаимопрозрачных самосогласованных сетей в условиях гравитации в ${ }^{L} S T$ и антигравитации в ${ }^{D} S T$ (см. п. 6.7). Именно, действием антигравитации удается объяснить солитонную природу формы антиматерии в пространстве-времени отрицательной кривизны ${ }^{D} S T$ (см. п. 6.8) и в результате, предложить солитонночастицную модель атомного ядра (и сильного взаимодействия, см. Главы 5 и 9).

В этой первой публикации, автор не стремился детально исследовать особенности каких-либо узких разделов физики (это будет сделано в последующих статьях: «Формализм TSSCU», «Особенности Калибровочной Теории и $C P T$-теоремы в $T S S C U »$ и других), возникающих в этой космологической модели, а ставил перед собою главную задачу оценить предлагаемую гипотезу на предмет отсутствия внутренней противоречивости, ее принципиальной работоспособности и перспективности. 
Несмотря, на выраженный дискуссионный характер предлагаемой работы, введение новой пространственно-временной симметрии в модели $B B-C r$ и построенная на этой основе теория стационарной самосогласованной Вселенной, позволяет поновому подойти к решению многих фундаментальных проблем космологии и современной физики. 


\section{Глава 2 Исходные положения TSSCU}

1. Вселенная одна (никаких параллельных и/или мульти Вселенных не существует).

2. Вселенная представляет собой две субстанции: первичное (материнское) Абсолютное пространство-время ${ }^{A} S T$ и Управляющую Супермнимую Единицу ${ }_{S} I$, объединяющую в себе, обобщенные комплексные числа с параболической $I$, гиперболической $\tilde{\imath}$ и эллиптической $i$ мнимыми единицами (см. пп. 3.5, 3.9, 3.10, Главы 3 и п. 4.2 Главы 4). Первичное (материнское) Абсолютное пространствовремя ${ }^{A} S T$ и Управляющая Супермнимая Единица ${ }_{s} I$ периодически взаимодействуют друг с другом (см. рис. 7), образуя обобщенное Паракомплексное Абсолютное пространство-время ${ }_{I}^{A} S T$ с параболической мнимой единицей $I$, в котором происходит Большой Взрыв $(B B)$ с последующим неотвратимым Схлопыванием $(\mathrm{Cr})$. Период, когда обе субстанции перестают взаимодействовать друг с другом (с момента $\mathrm{Cr}$ до очередного $\mathrm{BB}$ ) - является периодом Релаксации.

3. В момент $B B$ (см. рис. 1) в обобщенном Паракомплексном Абсолютном пространстве-времени ${ }_{I}^{A} S T$ (включающем в себя первичное (материнское) ${ }^{A} S T$ нулевой $(\Omega=0)$ и бесконечной $(\Omega=\infty)$ кривизны одновременно (см. п. 2 Главы $4)$, с геометрией Евклида $(\mathcal{E})$ и сферу с расстоянием-радиусом и кривизной, равными мнимой параболической единице $\left.I\left(S_{s p h}=I, \Omega_{s p h}=I\right)\right)$ реализуется пространственно-временная симметрия. Синхронно, то есть во времени синхронизации (далее - $T_{\text {synch }}$ ), рождаются два взаимопрозрачных пространствавремени:

- горячее пространство - время (далее - ${ }^{L} S T$ [Light (Real) Space - Time]) постоянной положительной кривизны $\left({ }^{L} \omega>0\right)$, с положительной плотностью материи ${ }^{L} M$ и энергии ${ }^{L} E$, стохастической информацией ${ }^{L} I$, в котором реализуется геометрия Римана $(\mathcal{R})$ эллиптическая, а ткань пространства однородно расширяется (относительно ткани Абсолютного пространствавремени $\left.{ }^{A} S T\right)$ во времени ${ }^{L} T$;

- холодное пространство - время (далее - ${ }^{D} S T$ [Dark (Hidden) Space - Time]) постоянной отрицательной кривизны $\left({ }^{D} \omega<0\right)$, с отрицательной плотностью материи ${ }^{D} M$ и энергии ${ }^{D} E$, фрактальной (или самоподобной, термин - требует точного научного определения) информацией ${ }^{D} I$, в котором реализуется геометрия Лобачевского-Больяи $(\mathcal{L B})$ гиперболическая, а ткань пространства неоднородно сжимается (относительно ткани Абсолютного пространствавремени $\left.{ }^{A} S T\right)$ во времени ${ }^{D} T$.

Пространства-времена ${ }^{L} S T$ и ${ }^{D} S T$ обладают следующими свойствами:

- являются взаимопрозрачными, то есть - не имеют возможности непосредственно взаимодействовать друг с другом;

- являются взаимосогласованными, так как вынуждены целенаправленно взаимодействовать друг с другом посредством обобщенного Паракомплексного Абсолютного пространства-времени ${ }_{I}^{A} S T$ (см. пункты 3.9 и 3.10 Главы 3); 
- являются фундаментально сцепленными друг с другом, то есть полностью взаимозависимыми (см. пункт 2.3 Главы 2 «Понятие фундаментальнойсцепленности - æ®);

- являются полными антиподами друг друга и имеют антагонистические свойства;

- являются квантованными, то есть фундаментальные взаимодействия в обоих пространствах-времени обладают квантами действия.

4. Пространства-времена ${ }^{L} S T$ и ${ }^{D} S T$ состоят из тканей (особых полевых форм) материи и антиматерии, развивающихся во временах ${ }^{L} T$ и ${ }^{D} T$, соответственно.

5. Во всех трех пространствах-времени ${ }^{A} S T,{ }^{L} S T$ и ${ }^{D} S T$ базисными категориями являются: Материя, Энергия, Информация.

6. В любой момент $T_{\text {synch }}$ суммарная плотность масс и плотность энергий двух пространств-времен ${ }^{L} S T$ и ${ }^{D} S T$ равна нулю.

7. Стохастическая информация ${ }^{L} I$ в ${ }^{L} S T$ и фрактальная информация ${ }^{D} I$ в ${ }^{D} S T$ являются взаимоисключающимися (антиподами друг друга) и при $\mathrm{Cr}$ в $T_{\text {synch }}$ взаимно нейтрализуют друг друга.

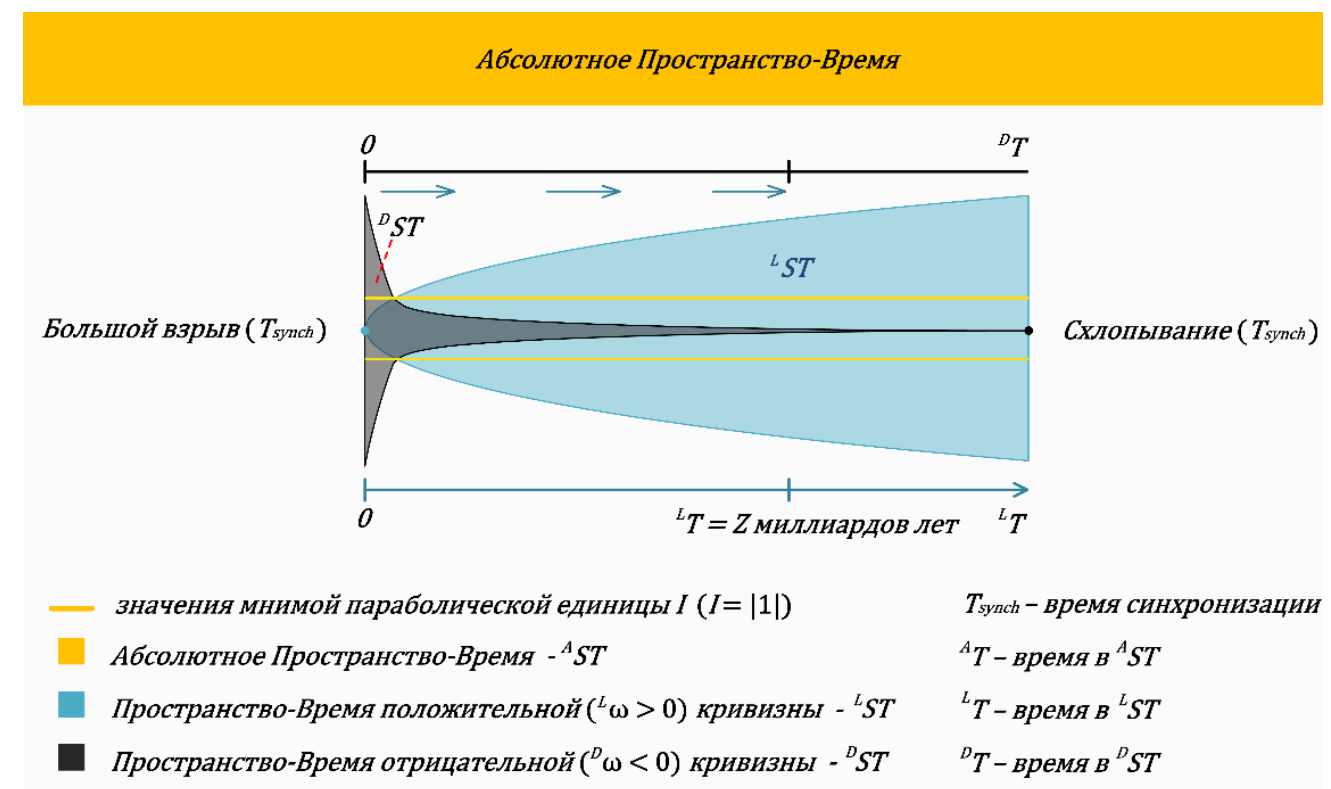

Рис. 1. Трех пространственно-временная модель строения Вселенной. В обобщенном Паракомплексном Абсолютном пространстве-времени ${ }_{I}^{A} S T$ в момент Большого Взрыва $(B B)$ синхронно (в $\left.T_{\text {synch }}\right)$ рождаются два взаимопрозрачных самосогласованных некоммутативных пространства-времени: постоянной положительной кривизны ${ }^{L} S T$ и постоянной отрицательной кривизны ${ }^{D} S T$. В любой момент времени синхронизации $T_{\text {synch }}$ произведение кривизны ${ }^{L} \omega$ пространствавремени ${ }^{L} S T$ на кривизну ${ }^{D} \omega$ пространства-времени ${ }^{D} S T$ равна мнимой параболической единице $I\left[{ }^{L} \omega\left(T_{\text {synch }}\right) \cdot{ }^{D} \omega\left(T_{\text {synch }}\right)=I\right]$, где $I=|\mathbf{1}|$. Судьба обоих пространств-времен ${ }^{L} S T$ и ${ }^{D} S T$ - предрешена, они исчезнут также синхронно (в $\left.T_{\text {synch }}\right)$, в момент Схлопывания $(\mathrm{Cr})$. 


\section{1 Понятие времени синхронизации $-T_{\text {synch }}$}

С одной стороны, у пространств ${ }^{L} S T$ и ${ }^{D} S T$ имеются собственные времена ${ }^{L} T$ и ${ }^{D} T$, имеющие свой темп и разные свойства. Но, с другой стороны, оба времени ${ }^{L} T$ и ${ }^{D} T$ порождаются из первичного вечного времени ${ }^{A} T$, процессом инициации $B B$, (cм. Главы 3 и 4). В результате все фундаментально сцепленные процессы в этих двух пространствах будут протекать синхронно во времени, которое так и назовем временем синхронизации $T_{\text {synch }}$.

\section{2 Понятие межпространственного потенциала - ${ }^{S T} U$}

В $T_{\text {synch }}$ в момент BB образовались: горячее пространство-время ${ }^{L} S T$ с очень высокой начальной температурой и холодное пространство-время ${ }^{D} S T$, вероятно, с температурой, эквивалентной абсолютному нулю (0K). Учитывая, разницу температур в момент рождения обоих пространств-времен ${ }^{L} S T$ и ${ }^{D} S T$, а также их антагонистические свойства, в обобщенном Паракомплексном Абсолютном пространстве-времени ${ }_{I}^{A} S T$ возникает межпространственный потенциал - ${ }^{S T} U$, благодаря которому реализуются все процессы от $B B$ до $C r$.

\section{3 Понятие фундаментальной сцепленности - æ}

Ниже приводится сокращенное определение понятия «фундаментальная сцепленность», полное и строгое определение будет дано в следующей статье «Формализм TSSCU».

Фундаментальная сцепленность - ǣ, (не следует путать с квантовой сцепленностью двух или нескольких объектов в пространстве-времени ${ }^{L} S T$, введенной в 1935 году в работе [59] и являющейся частным быстроисчезающим случаем фундаментальной сцепленности) это свойство парных объектов рожденных в $T_{\text {synch }}$ в пространствахвремени ${ }^{L} S T$ и ${ }^{D} S T$ (каждый объект рождается в своем пространстве-времени) сохранять полноценную зависимость между собою до преобразования в другие объекты или исчезновения, которые , также, обязательно произойдут в $T_{\text {synch }}$. 


\section{Глава 3 Первичное (материнское) Абсолютное пространство-время} ${ }^{A} S T$ как основа для модели Большого Взрыва $(B B)$ и последующего Схлопывания $(\mathrm{Cr})$

Прежде всего, попробуем сформулировать стоящую перед нами задачу, с учетом исходных положений, представленных в Главе 2.

\section{1 Цель и условия задачи}

Необходимо провести исследование по поиску алгоритмов (пусть, на первых порах, самых простейших и не полных), позволяющих смоделировать расслоение первичного (материнского) обобщенного Абсолютного пространства-времени ${ }^{A} S T$ на два пространства-времени ${ }^{L} S T$ и ${ }^{D} S T$, при условии стационарности ${ }^{A} S T$ и полного сохранения его целостности и индифферентности (условие самосогласованности) в любых процессах, происходящих в ${ }^{L} S T$ и ${ }^{D} S T$, в любой момент $T_{\text {synch }}$ с момента $B B$ и до $\mathrm{Cr}$.

Далее по тексту, для написания формул в ${ }^{A} S T$ мы будем использовать заглавные буквы (за исключением координат), а в ${ }^{L} S T$ и ${ }^{D} S T$ будут применяться прописные буквы (за исключением температуры и гравитации). В тех случаях, когда мнимая параболическая единица, обозначаемая как $I$, будет представлена численным значением, она будет обозначаться как 1 с полужирным начертанием.

\section{2 Базовые данные}

В ${ }^{A} S T$ реализуется геометрия Евклида $(\mathcal{E})$. Метрика в геометрии Евклида представлена формулой:

$$
S^{2}=\left(d x^{2}+d y^{2}+d z^{2}\right)
$$

Или, соответственно:

$$
S=\sqrt{d x^{2}+d y^{2}+d z^{2}}
$$

где, $S$ - расстояние или радиус сферы.

С учетом соотношения 2, связывающего расстояние, скорость и время:

$$
S=V \cdot T
$$

можно получить формулу 3:

$$
\sqrt{d x^{2}+d y^{2}+d z^{2}}=V \cdot T
$$

\section{3 Первое стартовое уравнение}

Для того, чтобы нам иметь возможность получить пространства-времена ${ }^{L} S T$ и ${ }^{D} S T$ постоянной положительной и постоянной отрицательной кривизны, в левую часть уравнения 3 нам необходимо добавить параметр кривизны сферы $\Omega$ с радиусом кривизны $S$, который равен:

$$
\Omega=\frac{1}{S^{2}}
$$


С учетом формулы 4 очевидно, что наиболее простым и приемлемым решением, подходящим для наших обозначенных целей и одновременно, позволяющим ввести в уравнение 3 параметр $\Omega$ - является сфера единичного радиуса, т.е. $S=1$ и, соответственно, $\Omega=1, V=1, T=1$.

Дополним формулу 3 параметром $\Omega$ :

$$
\Omega \cdot \sqrt{d x^{2}+d y^{2}+d z^{2}}=V \cdot T
$$

где, $\Omega=1, S=1, V=1, T=1$.

Уравнение 5 может служить стартовым для поиска верного алгоритма по расслоению ${ }^{A} S T$ и проведения необходимых нам дальнейших математических операций. Каждый, из рассматриваемых ниже алгоритмов может иметь одну или несколько сигнатур. Под понятием «сигнатура» мы будем понимать набор алгебраических и иных операций, которые необходимо совершить с уравнением, описывающим метрику пространства-времени (или с более расширенным уравнением), в результате применения которых мы получаем «совокупность свойств» первичного (материнского) пространства-времени ${ }^{A} S T$ и рожденных пространств-времен ${ }^{L} S T$ и ${ }^{D} S T$, которые нельзя отменить (они заданы в момент расслоения) и которые определяют условия развития и взаимодействия всех трех пространств-времен, начиная с момента $B B$ и до $C r$.

\section{4 Алгоритм 1. Умножение на мнимую единицу $[i]$}

Для начала выясним может ли ${ }^{D} S T$ состоять из мнимых координат и мнимого времени и отвечать требованиям предъявленными в главе 2. Несмотря на очевидность отрицательного ответа проведем простейшие операции, чтобы не возвращаться в будущем к этому вопросу. Для этого рассмотрим сигнатуру $\left[\cdot\left(1^{2}\right), \cdot(i), \leftrightarrow\right]$, где символ $\leftrightarrow$ будет означать расслоение.

Левую и правую часть уравнения 5 возведем в степень 2, а затем каждый сомножитель левой и правой части этого уравнения умножим на $i$. В результате получим:

$$
i \Omega^{2} \cdot i S^{2}=i V^{2} \cdot i T^{2}
$$

Представим уравнение 6 в виде:

$$
\Omega \cdot(i \Omega) \cdot S \cdot(i S)=V \cdot(i V) \cdot T \cdot(i T)
$$

Проведем операцию расслоения уравнения 7, представив его в виде двух некоммутативных уравнений 8L и 8D и выразив их через время:

Пространство-время ${ }^{L} S T$ :

$$
t=\frac{\omega \cdot \sqrt{\left(d x^{2}+d y^{2}+d z^{2}\right)}}{v}
$$

где, $t>0, \omega>0, v>0, s>0$ 
Пространство-время ${ }^{D} S T$ :

$$
i t=\frac{i \omega \cdot i \sqrt{\left(d x^{2}+d y^{2}+d z^{2}\right)}}{i v}
$$

Мы получили пространство-время положительной $\quad$ кривизны ${ }^{L} S T$ с 3 действительными координатами и 1 временем (4 измерения) и в ${ }^{D} S T$ мнимую координату (значение которой зависит от 3-х действительных координат) с мнимым временем. Очевидно, что заявленного в главе 2, полноценного (4 измерения) пространства-времени ${ }^{D} S T$ мы не получили, следовательно, сигнатура $\left[\cdot\left(1^{2}\right), \cdot(i), \leftrightarrow\right]-$ не верна.

Рассмотрим сигнатуру [ $\left.\left(1^{4}\right), \cdot(i), \leftrightarrow\right]$. Левую и правую часть уравнения 5 возведем в степень 4, а затем каждый сомножитель левой и правой части умножим на $i$.

В результате получим:

$$
i \Omega^{4} \cdot i S^{4}=i V^{4} \cdot i T^{4}
$$

Представим уравнение 9 в виде:

$$
\Omega^{2} \cdot\left(i \Omega^{2}\right) \cdot S^{2} \cdot\left(i S^{2}\right)=V^{2} \cdot\left(i V^{2}\right) \cdot T^{2} \cdot\left(i T^{2}\right)
$$

Проведем операцию расслоения уравнения 10, представив его в виде двух некоммутативных уравнений 11L и 11D и выразив их через время:

Пространство-время ${ }^{L} S T$ :

$$
t^{2}=\frac{\omega^{2} \cdot\left(d x^{2}+d y^{2}+d z^{2}\right)}{v^{2}}
$$

где, $t>0, \omega>0, v>0, s>0$

Пространство-время ${ }^{D} S T$ :

$$
i t^{2}=\frac{i \omega^{2} \cdot\left(i d x^{2}+i d y^{2}+i d z^{2}\right)}{i v^{2}}
$$

Мы получили пространство-время положительной $\quad$ кривизны ${ }^{L} S T \quad$ c 3 действительными координатами и 1 временем (4 измерения) и некое пространствовремя с 3 мнимыми координатами и 1 мнимым временем. В данном случае мы даже не будем анализировать корректность чисто мнимых координат с мнимым временем, поскольку, полученные уравнения 11L и 11D противоречат пунктам (3-7) исходных положений главы 2, и, следовательно, примененная сигнатура $\left[\cdot\left(1^{4}\right), \cdot(i), \leftrightarrow\right]$ - также неверна.

Вывод: Пространство-время ${ }^{D} S T$ - не является пространством с мнимыми координатами и мнимым временем, а алгоритм 1 - неработоспособен.

\section{5 Алгоритм 2. Возведение в степень и умножение на отрицательную единицу [-1]}

Сигнатура $\left[\cdot 1^{2}, \cdot(-1), \leftrightarrow\right]$. Левую и правую часть уравнения 5 возведем в степень 2 и каждый сомножитель левой и правой части умножим на (-1). В результате получим:

$$
-\Omega^{2} \cdot-S^{2}=-V^{2} \cdot-T^{2}
$$


Представим уравнение 12 в виде:

$$
\Omega \cdot(-\Omega) \cdot S \cdot(-S)=V \cdot(-V) \cdot T \cdot(-T)
$$

Проведем операцию расслоения уравнения 13, представив его в виде двух самосогласованных некоммутативных уравнений 14L и 14D и выразив их через время:

Пространство-время ${ }^{L} S T$ :

$$
t=\frac{\omega \cdot \sqrt{d x^{2}+d y^{2}+d z^{2}}}{v}
$$

где, $t>0, \omega>0, s>0, v>0$

Пространство-время ${ }^{D} S T$ :

$$
-t=\frac{-\omega \cdot-\sqrt{d x^{2}+d y^{2}+d z^{2}}}{-v}
$$

Уравнения 14L и 14D, в первом приближении, являются удовлетворительными и определяют параметры, а также некоторые характеристики дальнейшего развития пространств-времен положительной и отрицательной кривизны, соответственно. Единственным, не до конца определенным - является сомножитель: $-\sqrt{d x^{2}+d y^{2}+d z^{2}}$, входящий в уравнения 14D. Попробуем разобраться с ним, и понять, каким образом, в пространстве-времени ${ }^{D} S T$ могут быть представлена координатная система. Рассмотрим два варианта. Первый вариант с положительноотрицательной симметрией значений координат представлен на рис. 2, который, фактически, следует из интерпретации автором работы Р. Ф. Фейнмана [60], косвенно характеризующей некоторые свойства ${ }^{D} S T$. Второй вариант с положительноотрицательной симметрией направлений осей координат, представлен на рис. 3.

Пространство-время ${ }^{L} S T$

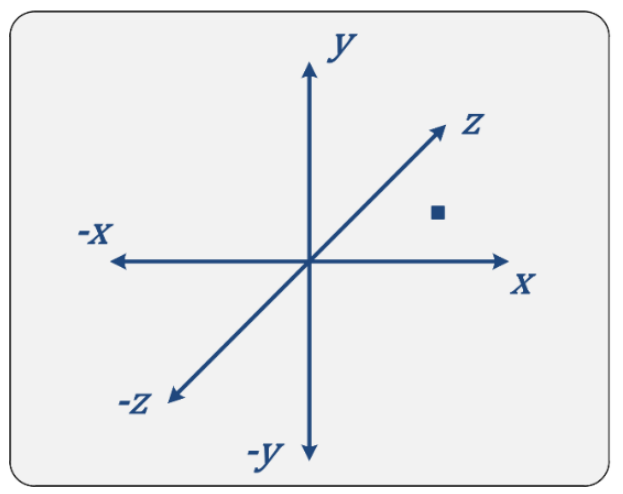

Пространство-время ${ }^{D} S T$

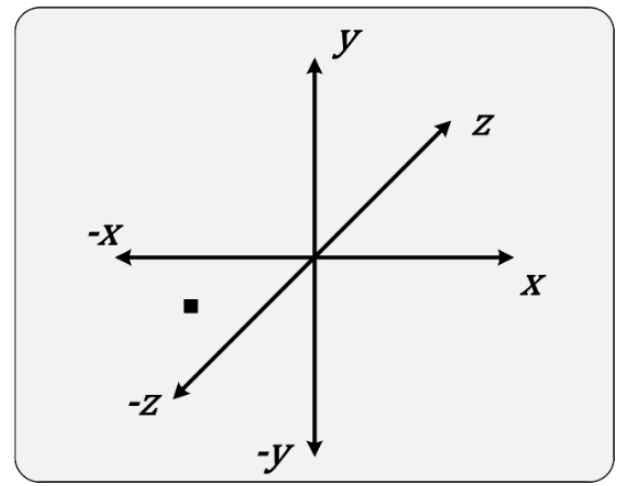

Рис. 2. Сопоставление прямоугольных (декартовых) систем координат в пространствах-времени ${ }^{L} \boldsymbol{S} \boldsymbol{T}$ и ${ }^{\boldsymbol{D}} \boldsymbol{S T}$ - первый вариант. В левой части представлена, привычная в математике, прямоугольная система координат в пространствевремени ${ }^{L} S T$. В правой части представлена гипотетическая прямоугольная система координат в пространстве-времени ${ }^{D} S T$, при которой положительные оси координат в ${ }^{L} S T$ и ${ }^{D} S T$, а также отрицательные оси координат в ${ }^{L} S T$ и ${ }^{D} S T$ направлены в одну и ту же сторону (совпадают). 
Пространство-время ${ }^{L} S T$

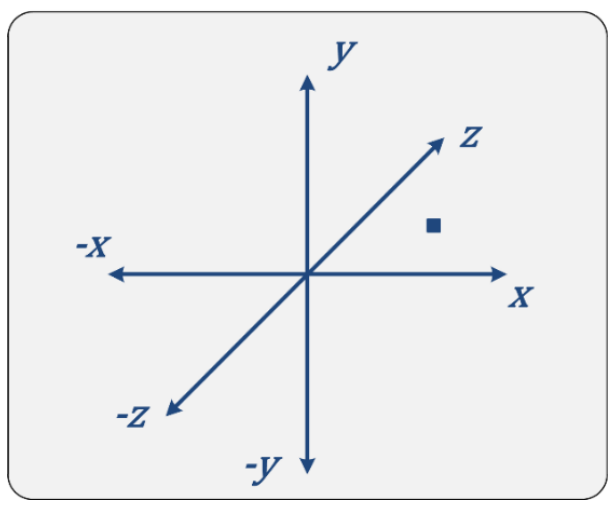

Пространство-время ${ }^{D} S T$

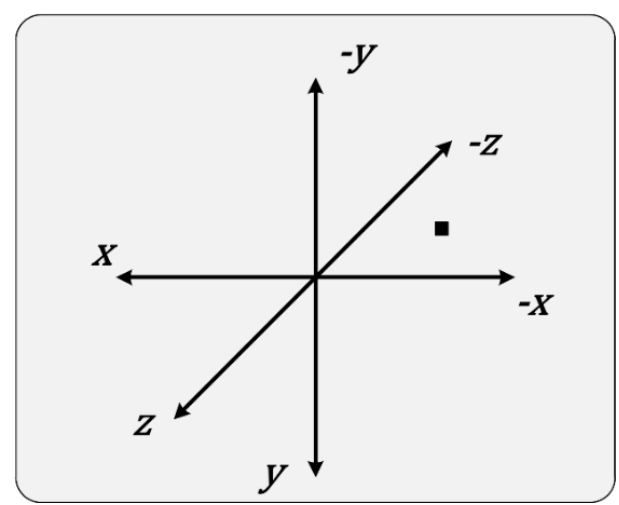

Рис. 3. Сопоставление прямоугольных (декартовых) систем координат в пространствах-времени ${ }^{L} \boldsymbol{S T}$ и ${ }^{D} \boldsymbol{S T}$ - второй вариант. В левой части представлена, привычная в математике, прямоугольная система координат в пространствевремени ${ }^{L} S T$. В правой части представлена гипотетическая прямоугольная система координат в пространстве-времени ${ }^{D} S T$, при которой положительные оси координат в ${ }^{L} S T$ и ${ }^{D} S T$, а также отрицательные оси координат в ${ }^{L} S T$ и ${ }^{D} S T$ направлены в противоположные стороны.

С учетом того, что пространства-время ${ }^{L} S T$ и ${ }^{D} S T$, согласно пункту 3 Главы 2 являются полными антиподами друг друга, мы можем предположить, что вариант, с положительно-отрицательной симметрией направления осей координат, представленный на рис. 3 - больше соответствует нашей цели. Именно, этот вариант с положительно-отрицательной симметрией направления осей координат и был применен при создании рис. 1.

Для того, чтобы нам разобраться с полученными уравнениями 14L и 14D необходимо отметить, что при расслоении ${ }^{A} S T$ в пространство-время ${ }^{L} S T$ делегируется (по умолчанию) привычный для нас элемент сигнатуры $\left[+1^{+1}\right]$, что означает (плюс единица в степени плюс единица), который будем называть абсолютным доминантным плюсом и обязательно указывать в сигнатурах при дальнейших исследованиях. А, в пространство-время ${ }^{D} S T$ делегируется элемент сигнатуры [-1], который будем называть доминантным минусом.

На основании вышесказанного, к привычной для нас алгебре с абсолютным доминантным плюсом, применяемой в ${ }^{L} S T$ мы можем ввести положительноотрицательную симметричную алгебру с доминантным минусом в пространствевремени ${ }^{D} S T$. Для этого, зададим соответствующие правила операций сложения, умножения и других элементов алгебры для «темных» чисел (назовем их так, чтобы не путать с обычными отрицательными числами и будем обозначать их двумя первыми латинскими буквами: $D a$ ) в пространстве-времени ${ }^{D} S T$. Сопоставим эти правила операций для множества действительных чисел $(\mathbb{R})$ в ${ }^{L} S T$ и множества «темных» чисел $(\mathbb{D})$ в ${ }^{D} S T$ в табл. 1. 
Таблица 1. Сопоставление правил математических операций для действительных чисел $(R e)$, получаемых с использованием элемента сигнатуры $\left[+1^{+1}\right]$ в ${ }^{L} S T$ и темных чисел $(D a)$, получаемых с использованием элемента сигнатуры $[-1]$ в ${ }^{D} S T$

\begin{tabular}{|c|c|}
\hline Пространство-время ${ }^{L} S T$ & Пространство-время ${ }^{D} S T$ \\
\hline Элемент сигнатуры $\left[+1^{+1}\right]$ & Элемент сигнатуры [-1] \\
\hline Действительные числа $(R e): a, b, c, d \ldots$ & Темные числа $(D a):-\widetilde{a},-\widetilde{b},-\widetilde{c},-\tilde{d} \ldots$ \\
\hline$+a>0>-a$ & $-\tilde{a}>0>+\widetilde{a}$ \\
\hline $\begin{array}{l}\text { Операция сложения: } \\
\qquad \begin{array}{r}+a+b=+c \\
-a+b= \pm c\end{array}\end{array}$ & $\begin{array}{l}\text { Операция сложения: } \\
\qquad \begin{aligned}-\tilde{a}-\tilde{b} & =-\tilde{c} \\
+\tilde{a}-\tilde{b} & = \pm \tilde{c}\end{aligned}\end{array}$ \\
\hline $\begin{array}{l}\text { Операция умножения: } \\
\qquad \begin{array}{r}(+a) \cdot(+b)=+c \\
(+a) \cdot(-b)=-c \\
(-a) \cdot(-b)=+c\end{array}\end{array}$ & $\begin{array}{l}\text { Операция умножения: } \\
\qquad \begin{aligned}(-\tilde{a}) \cdot(-\tilde{b}) & =-\tilde{c} \\
(-\tilde{a}) \cdot(+\tilde{b}) & =+\tilde{c} \\
(+\tilde{a}) \cdot(+\tilde{b}) & =-\tilde{c}\end{aligned}\end{array}$ \\
\hline $\begin{array}{l}\text { Возведение в степень: } \\
\qquad a^{2}=+c\end{array}$ & $\begin{array}{l}\text { Возведение в степень: } \\
\qquad \pm \tilde{a}^{2}=-\tilde{c}\end{array}$ \\
\hline $\begin{array}{c}\text { Извлечение из квадратного корня: } \\
\sqrt{+a}=+c\end{array}$ & $\begin{array}{c}\text { Извлечение из квадратного корня: } \\
\sqrt{-\tilde{a}}=-\tilde{c}\end{array}$ \\
\hline $\begin{array}{r}\text { Комплексные числа: } \\
\qquad \begin{array}{r}a+i b \\
i^{2}=-1\end{array}\end{array}$ & $\begin{array}{c}\text { Темные комплексные числа: } \\
\qquad \begin{array}{c}-\tilde{a}-\tilde{\imath} \tilde{b} \\
\tilde{l}^{2}=1\end{array}\end{array}$ \\
\hline $\begin{array}{l}\text { Кватернионы: } \\
\qquad \begin{array}{l}q=a+b i+c j+d k \\
\quad i^{2}=j^{2}=k^{2}=i j k=-1\end{array} \\
\text { и так далее... }\end{array}$ & $\begin{array}{l}\text { Темные кватернионы: } \\
\qquad-\tilde{q}=-\tilde{a}-\tilde{b} \tilde{\imath}-\tilde{c} \tilde{j}-\tilde{d} \tilde{k} \\
\quad \tilde{l}^{2}=\tilde{\jmath}^{2}=\tilde{k}^{2}=\tilde{\imath} \tilde{j}=1 \\
\text { и так далее... }\end{array}$ \\
\hline
\end{tabular}

Если предположить, что такая алгебра с доминантным минусом реализуется в ${ }^{D} S T$, то из табл. 1 становится понятным (раскрывается) смысл происхождение мнимой эллиптической единицы $i$, квадрат которой $i^{2}=-1$ в теории функции комплексной переменной (ТФКП). Такое умножение полностью соответствует правилам умножения темных чисел в ${ }^{D} S T$. С учетом, полученных выводов можно попробовать дать новую предварительную интерпретацию, расширенное определение ТФКП. Это удобный и мощный математический инструмент, предоставляющий возможность быстро и эффективно решать определенного класса задачи в ${ }^{L} S T$. Но, одновременно, ТФКП - это еще и локальная и ограниченная «модель-мост», объединяющая свойства привычной для нас алгебры с абсолютным доминантным плюсом и алгебры с доминантным минусом. Такое локальное объединение на основе множества 
комплексных чисел $(\mathbb{C})$ позволяет описывать некоторые аспекты взаимодействий объектов в пространствах-времени ${ }^{L} S T$ и ${ }^{D} S T$ (например, уравнение Шредингера см. главу 11). При этом комплексные числа включают в себя, как действительные числа, так и мнимые, наделенные свойствами темных чисел в ${ }^{D} S T$. Как видно из второй колонки табл. 1, в ${ }^{D} S T$ можно пойти аналогичным путем и ввести темную мнимую гиперболическую единицу $\tilde{\imath}$, квадрат которой равен $\tilde{l}^{2}=1$, темные комплексные числа и т.д. Вопрос о природе комплексных чисел мы будем подробнее рассматривать в п. 3.9 настоящей Главы, а пока вернемся к не определенному сомножителю: $-\sqrt{d x^{2}+d y^{2}+d z^{2}}$. Его в алгебре с доминантным минусом можно написать, как: $\sqrt{-d x^{2}-d y^{2}-d z^{2}}$, поскольку квадратный корень из отрицательного числа будет отрицательным числом. Соответственно, уравнение $14 \mathrm{D}$ будет выглядеть следующим образом:

$$
-t=\frac{-\omega \cdot \sqrt{-d x^{2}-d y^{2}-d z^{2}}}{-v}
$$

а, параметры $-t,-\omega,-s,-v>0$ в ${ }^{D} S T$.

Вывод: алгоритм 2 с сигнатурой $\left[\cdot 1^{2}, \cdot\left(+1^{+1}\right), \cdot(-1), \leftrightarrow\right]-$ является, в первом приближении, удовлетворительным и требует дальнейших исследований, а уравнение 14D относится к алгебре с доминантным минусом.

\section{6 Гравитация и температура. Основное стартовое уравнение}

Попытаемся добавить в уравнение 5 еще два необходимых параметра: гравитацию $G_{r}$ и температуру $K$. С учетом специфики этого уравнения оба параметра в ${ }^{A} S T$ с геометрией Евклида $(\mathcal{E})$ в момент $B B$ и до $C r$, также должны быть равны 1.

Гравитация ${ }^{A} G_{r}=1$

Температура ${ }^{A} K=1$

Нам необходимо определить в какую часть (левую или правую) уравнения 5 мы вправе расположить оба параметра, с точки зрения стартовых условий и динамики развития пространств-времен ${ }^{L} S T$ и ${ }^{D} S T$ (см. рис. 1).

Начнем с гравитации $G_{r}$. Она в ${ }^{L} S T$ представляет собою силу, которая возникает как противодействующая (стремящаяся компенсировать) расширению этого пространства, и соответственно, изменению двух обратнозависимых параметров: расстояния-радиуса кривизны - ${ }^{L} S$ в сторону увеличения и кривизны - ${ }^{L} \omega$ в сторону уменьшения. Следовательно, параметры ${ }^{L} S$ и ${ }^{L} G_{r}$ находятся между собою в прямой зависимости. Чем больше расстояние-радиус кривизны ${ }^{L} S$, тем больше гравитация ${ }^{L} G_{r}$. И, наоборот, чем больше гравитация ${ }^{L} G_{r}$, тем больше расстояние-радиус кривизны ${ }^{L} S$. Очевидно, что в этом случае параметр $G_{r}$ необходимо разместить в правой части уравнения 5 .

Температура ${ }^{L} K_{1}$ в первый момент $B B$ - максимальна в пространстве-времени ${ }^{L} S T$, в то время как, параметр ${ }^{L} S_{1}$ - минимален. А в пространстве-времени ${ }^{D} S T$ : все наоборот. Второй критерий: чем больше температура $K$ в ${ }^{L} S T$, тем больше должна быть скорость $V$. Следовательно, параметр $K$ необходимо разместить в левой части уравнения 5. 
В результате, для ${ }^{A} S T$ получаем уравнение 15 :

$$
K \cdot \Omega \cdot S=V \cdot T \cdot G_{r}
$$

Напомним, что $K$ - это температура, $\Omega$-кривизна пространства, $S$ - расстояние или радиус сферы, $V$ - скорость, $T$ - время, $G_{r}$ - гравитация.

Уравнение 15 может служить основным стартовым уравнением, для проведения необходимых дальнейших математических операций по расслоению ${ }^{A} S T$.

Если с уравнением 15 мы проведем аналогичные операции, как в пункте 3.5 с сигнатурой $\left[\cdot 1^{2}, \cdot\left(+1^{+1}\right), \cdot(-1), \leftrightarrow\right]$, но с учетом трех множителей слева и трех справа, то мы получим два самосогласованных некоммутативных уравнения 16L и 16D, каждое из которых определяет начальные условия и некоторые динамические характеристики дальнейшего развития пространств-времен положительной и отрицательной кривизны, соответственно.

Пространство-время ${ }^{L} S T$ :

$$
t=\frac{K \cdot \omega \cdot \sqrt{d x^{2}+d y^{2}+d z^{2}}}{G_{r} \cdot v}
$$

где, $t>0, K>0, \omega>0, s>0, G_{r}>0, v>0$

В полученном уравнении 16L привычное для нас положительное время ${ }^{L} t$ имеет, не только предсказанную обратную зависимость от гравитации $G_{r}$ и скорости $v[61,62$, $63,64,65,66]$, но и прямую от температуры $K$, кривизны пространства $\omega$ и расстояниярадиуса $s$.

Пространство-время ${ }^{D} S T$ :

$$
-t=\frac{-K \cdot-\omega \cdot \sqrt{-d x^{2}-d y^{2}-d z^{2}}}{-G_{r} \cdot-v}
$$

где, $-t>0,-K>0,-\omega>0,-s>0,-G_{r}>0,-v>0$.

Уравнение 16D относится к алгебре с доминантным минусом.

Вывод: полученное уравнение 15 - является основным стартовым уравнением и требует дальнейших исследований.

\section{7 Алгоритм 3. Обратная зависимость с элементом сигнатуры $\left[-1^{-1}\right]$}

Для решения поставленной нами задачи, рассмотрим возможность реализации алгоритма с обратной зависимостью с сигнатурой $\left[\cdot 1^{2}, \cdot\left(+1^{+1}\right), \cdot\left(-1^{-1}\right), \leftrightarrow\right]$. Элемент сигнатуры $\left[-1^{-1}\right]$ будем называть абсолютным доминантным минусом (минус единица в степени минус единица). Левую и правую часть уравнения 15 возведем в степень 2 и представим в виде:

$$
K \cdot(K) \cdot \Omega \cdot(\Omega) \cdot S \cdot(S)=V \cdot(V) \cdot T \cdot(T) \cdot G_{r} \cdot\left(G_{r}\right)
$$

Каждый сомножитель левой и правой части, находящийся в скобках, умножим на $\left(-1^{-1}\right)$. В результате получим: 


$$
K \cdot\left(-\frac{1}{K}\right) \cdot \Omega \cdot\left(-\frac{1}{\Omega}\right) \cdot S \cdot\left(-\frac{1}{S}\right)=V \cdot\left(-\frac{1}{V}\right) \cdot T \cdot\left(-\frac{1}{T}\right) \cdot G_{r} \cdot\left(-\frac{1}{G_{r}}\right)
$$

Проведем операцию расслоения уравнения 18, представив его в виде двух самосогласованных некоммутативных уравнений 19L и 19D и выразим их через время:

Пространство-время ${ }^{L} S T$ :

$$
t=\frac{K \cdot \omega \cdot\left(d x^{2}+d y^{2}+d z^{2}\right)}{G_{r} \cdot v}
$$

где, $t>0, K>0, \omega>0, s>0, G_{r}>0, v>0$

Пространство-время ${ }^{D} S T$ :

$$
-\frac{1}{t}=\frac{-\frac{1}{K} \cdot-\frac{1}{\omega} \cdot \frac{-1}{\sqrt{-d x^{2}-d y^{2}-d z^{2}}}}{-\frac{1}{G_{r}} \cdot-\frac{1}{v}}
$$

Напишем уравнение 19D в более компактном виде:

$$
-t^{-1}=\frac{-K^{-1} \cdot-\omega^{-1} \cdot-s^{-1}}{-G_{r}^{-1} \cdot-v^{-1}}
$$

где, $-t^{-1}>0,-K^{-1}>0,-\omega^{-1}>0,-s^{-1}>0,-G_{r}^{-1}>0,-v^{-1}>0$, а уравнения 19D (или 20D) относятся к алгебре с абсолютным доминантным минусом.

Проанализируем полученные результаты с использованием рис. 4. и табл. 2. 


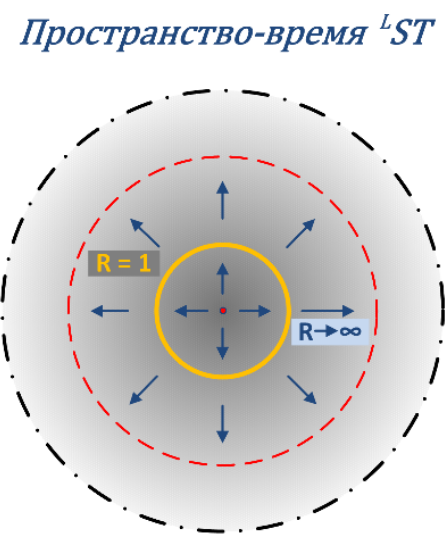

Сфера единичного радиуса $-{ }^{A} S T$

Пространство-Время положительной кривизны $-{ }^{L} S T$

Пространство-Время отрицательной кривизны $-{ }^{D} S T$
Пространство-время ${ }^{D} S T$

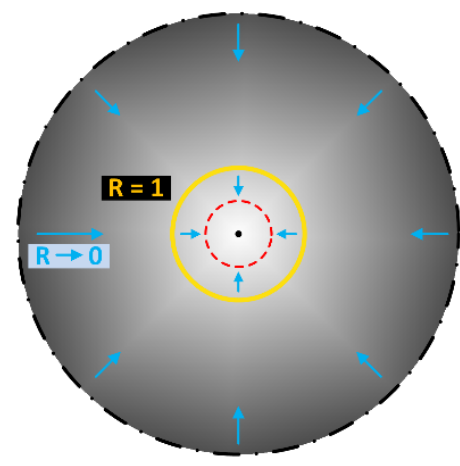

- Окрестность нуля

?. Бесконечность

,- Современный этап развития Вселенной

Рис. 4. Плоская обобщенная модель Большого Взрыва $(B B)$ и последующего Схлопывания $(\mathrm{Cr})$ в поперечном разрезе, которое служит дополнением к модели, представленной на рис. 1.

Таблица 2. Сопоставление некоторых свойств пространств-времен ${ }^{L} S T$ и ${ }^{D} S T$ в момент их рождения $(B B)$, этапность развития и последующего Схлопывания $(C r)$ с учетом обратной зависимости обоих пространств-времен между собой.

\begin{tabular}{|c|c|}
\hline Пространство-время ${ }^{L} S T$ & Пространство-время ${ }^{D} S T$ \\
\hline $\begin{array}{l}B B \text { начинается в радиусе отличном от } \\
\text { нуля }\left({ }^{L} r>0\right) \text { на бесконечно малую } \\
\text { величину и распространяется во вне } \\
\text { (расширение) во все стороны, } \\
\text { приближаясь к значению единичного } \\
\text { радиуса }\left({ }^{L} r \approx 1\right), \text { и далее стремится к } \\
\text { радиусу, стремящемуся к бесконечности } \\
\left({ }^{L} r \rightarrow \infty\right) \text { до } C r . \\
\text { Как будет ясно в дальнейшем (см. п. } \\
3.10) \text { значение }{ }^{L} r=1 \text { синхронное со } \\
\text { значением }{ }^{D} r=-1 \text { в }{ }^{D} S T \text { невозможно. } \\
\text { Можно выделить } 3 \text { основных этапа для } \\
\text { детальных исследований: } \\
\text { • } 1 \text {-й этап: } 0<{ }^{L} r<1 \\
\text { - } 2 \text {-й этап: } 1<{ }^{L} r<\infty \\
\text { - } 3 \text {-й этап: }{ }^{L} r \text { находится вблизи } \\
\text { сингулярностей: около } 0 \text { и } \infty .\end{array}$ & 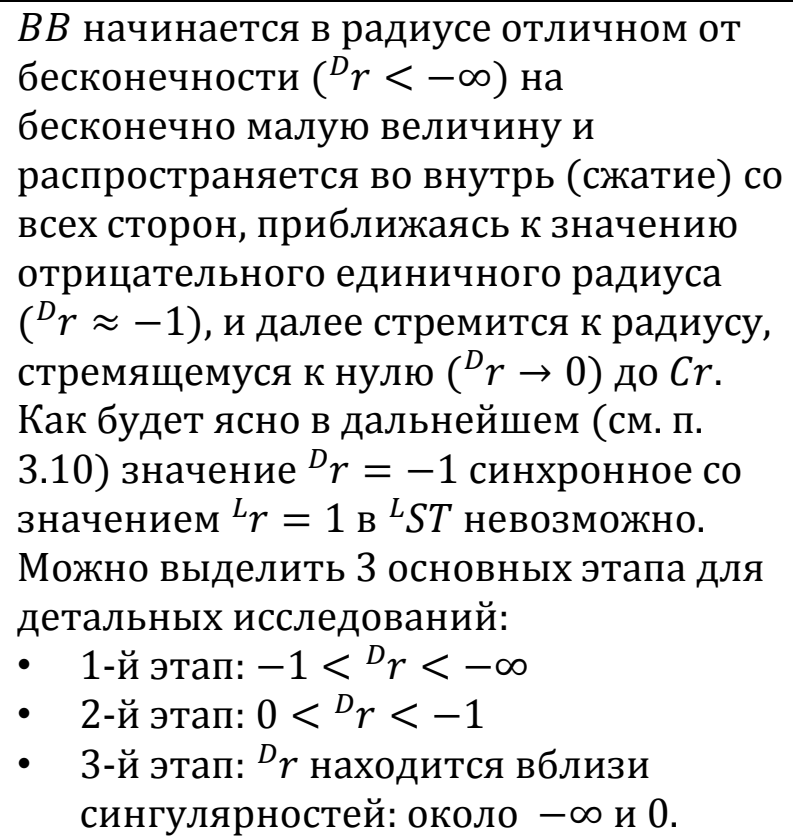 \\
\hline
\end{tabular}


Для проведения исследований сингулярностей, обозначенных на 3-ем этапе в обоих пространствах-времени ${ }^{L} S T$ и ${ }^{D} S T$ можно воспользоваться, в частности, инфинитезимальным (нестандартным) анализом $[67,68,69]$. Одним из критериев эффективности инфинитезимального анализа (как, впрочем, и любого другого) в обоих пространствах-времени должно быть объяснение численного значения скорости света: $c$ в ${ }^{L} S T$, и соответственно, усредненной скорости темного света: $-\frac{1}{c}$ в ${ }^{D} S T$. Важно помнить, что в любой момент $T_{\text {synch }}$ выполняется условия уравнений $19 \mathrm{~L}$ и $19 \mathrm{D}$.

Равносильность пространств-времен ${ }^{L} S T$ и ${ }^{D} S T$, а также тот факт, что они являются антиподами друг к другу, приводит к удивительному парадоксу приоритета точки отсчета (или точек наблюдения). Рассмотрим начальные значения параметров уравнения 19L и 19D с точки зрения деления их на максимумы и минимумы. Полученные данные представим в таблице 3 , учитывая выбор приоритетности точек отсчета и элементов сигнатуры в ${ }^{L} S T$ и ${ }^{D} S T$.

Табл. 3 Начальные значения шести пар базовых параметров, входящих в уравнения 19L и 19D с точки зрения деления их на максимумы и минимумы, а также выбора приоритета точки отсчета. Эти максимумы и минимумы - прямо противоположны друг другу и зависят от того какое пространствовремя ${ }^{L} S T$ или ${ }^{D} S T$ является приоритетным для исследователя. Такая субъективность - приводит к парадоксу выбора приоритета точки отсчета (наблюдения).

\begin{tabular}{|c|c|c|c|}
\hline \multicolumn{2}{|c|}{ Приоритет точки отсчета в ${ }^{L} S T$} & \multicolumn{2}{|c|}{ Приоритет точки отсчета в ${ }^{D} S T$} \\
\hline \multicolumn{2}{|c|}{ Элемент сигнатуры $\left[+1^{+1}\right]$} & \multicolumn{2}{|c|}{ Элемент сигнатуры $\left[-1^{-1}\right]$} \\
\hline${ }^{L} K_{0}-\max$ & ${ }^{D} K_{0}-\min$ & ${ }^{L} K_{0}-\min$ & ${ }^{D} K_{0}-\max$ \\
\hline${ }^{L} \omega_{0}-\max$ & ${ }^{D} \omega_{0}-\min$ & ${ }^{L} \omega_{0}-\min$ & ${ }^{D} \omega_{0}-\max$ \\
\hline${ }^{L} s_{0}-\min$ & ${ }^{D} S_{0}-\max$ & ${ }^{L} S_{0}-\max$ & ${ }^{D} S_{0}-\min$ \\
\hline${ }^{L} t_{0}-\min$ & ${ }^{D} t_{0}-\max$ & ${ }^{L} t_{0}-\max$ & ${ }^{D} t_{0}-\min$ \\
\hline${ }^{L} v_{0}-\max$ & ${ }^{D} v_{0}-\min$ & ${ }^{L} v_{0}-\min$ & ${ }^{D} v_{0}-\max$ \\
\hline${ }^{L} G r_{0}-\min$ & ${ }^{D} G r_{0}-\max$ & ${ }^{L} G r_{0}-\max$ & ${ }^{D} G r_{0}-\min$ \\
\hline
\end{tabular}

Этот парадокс выбора приоритета точки отсчета - требует отдельного специального рассмотрения. В данной статье, при столкновении с ним, мы будем исходить из условий стоящей перед нами задачи (напр. см. п. 6.7 Главы 6,). Но важно отметить, что если для исследователя приоритетным является исходное первичное (материнское) Абсолютное пространство-время ${ }^{A} S T$, то этот парадокс находится под контролем.

Вывод: уравнение 15 , сигнатура $\left[\cdot 1^{2}, \cdot\left(+1^{+1}\right), \cdot\left(-1^{-1}\right), \leftrightarrow\right]$ и полученные на их основе уравнения 19L и 19D - являются перспективными и основными, однако требуют дальнейших исследований и расширения. Смысл этих уравнений будет, не раз, нами обсуждаться по ходу этой статьи. 


\section{8 Алгоритмы и частичное расслоение Абсолютного пространства-времени ${ }^{A} S T$}

В предыдущих примерах мы рассматривали случаи полного расслоения Абсолютного пространства-времени ${ }^{A} S T$. Но существует также возможность частичного расслоения Абсолютного пространства-времени ${ }^{A} S T$, что может, например, существенно влиять на соотношения плотностей тканей пространств-времен (и, соответственно, соотношения плотностей видов материй и энергий) во Вселенной.

Для этого, например, можно использовать сигнатуру $\left[\cdot 1^{4}, \cdot\left(+1^{+1}\right), \cdot\left(-1^{-1}\right), \leftrightarrow\right]$. Левую и правую часть уравнения 15 возведем в степень 4 и представим в следующем виде:

$$
K^{2} \cdot K \cdot(K) \cdot \Omega^{2} \cdot \Omega \cdot(\Omega) \cdot S^{2} \cdot S \cdot(S)=V^{2} \cdot V \cdot(V) \cdot T^{2} \cdot T \cdot(T) \cdot G_{r}{ }^{2} \cdot G_{r} \cdot\left(G_{r}\right)
$$

Каждый сомножитель левой и правой части уравнения 21, находящийся в скобках, умножим на $\left(-1^{-1}\right)$. В результате получим:

$$
K^{2} \cdot K \cdot\left(-\frac{1}{K}\right) \cdot \Omega^{2} \cdot \Omega \cdot\left(-\frac{1}{\Omega}\right) \cdot S^{2} \cdot S \cdot\left(-\frac{1}{S}\right)=V^{2} \cdot V \cdot\left(-\frac{1}{V}\right) \cdot T^{2} \cdot T \cdot\left(-\frac{1}{T}\right) \cdot G_{r}^{2} \cdot G_{r} \cdot\left(-\frac{1}{G_{r}}\right)
$$

С уравнением 22 проведем операцию частичного расслоения. В результате получим: в Абсолютном пространстве-времени ${ }^{A} S T$ формулу:

$$
K^{2} \cdot \Omega^{2} \cdot S^{2}=V^{2} \cdot T^{2} \cdot G_{r}^{2}
$$

в пространстве-времени ${ }^{L} S T$ формулу $19 \mathrm{~L}$, а в пространстве-времени ${ }^{D} S T$ формулу $19 \mathrm{D}$.

Вывод: при частичном расслоении Абсолютного пространства-времени ${ }^{A} S T$ вклад ${ }^{L} S T$ ${ }^{D}{ }^{D} T$ в общую плотность материй и энергий во Вселенной существенно снижается, и соответственно, требуется точное определение соотношений между плотностями ${ }^{A} S T$ и $\left({ }^{L} S T\right.$ и $\left.{ }^{D} S T\right)$ вместе взятых.

\section{9 Алгоритмы и Управляющая Супермнимая Единица ${ }_{S} I$}

Главный вопрос, на который нам предстоит ответить в этом разделе это каким образом два взаимопрозрачных некоммутативных пространства-времени ${ }^{L} S T$ и ${ }^{D} S T-$ являются самосогласованными? Начнем с того обстоятельства, что $B B$ произошел не в одной центральной точке, а в бесконечном количестве точек во Вселенной. И, соответственно, в обоих пространствах-времени в момент $B B$ родилось бесконечное количество фундаментально-сцепленных, точек-центров в ${ }^{L} S T$ и бесконечностей в ${ }^{D} S T$, которые взаимодействуют друг с другом через обобщенное Паракомплексное Абсолютное пространство-время ${ }_{I}^{A} S T$ и образуют доминантные области развития (самосогласованное однородное расширение ${ }^{L} S T$ и неоднородное сжатие ${ }^{D} S T$ ). Что из себя представляет это обобщенное Паракомплексное Абсолютное пространствовремя ${ }_{I}^{A} S T$ ? Это исключительно первичное (материнское) Абсолютное пространствовремя ${ }^{A} S T$ ? Или, это Управляющая мнимая субстанция, неразрывно связанное с ${ }^{A} S T$ в период с $B B$ до $C r$ и образующее вместе с ним - обобщенное Паракомплексное ${ }_{I}^{A} S T$ ? Попробуем разобраться в этих важнейших вопросах. 
Прежде всего, хочется отметить, что появление квадратного корня в уравнении 1, задающей метрику в ${ }^{A} S T$ - неслучайно. Видимо, уже на этом самом первом этапе, мы получили намек на комплексную природу чисел в ${ }^{L} S T$ и темную комплексную природу чисел в ${ }^{D} S T$, а также Паракомплексную природу первичных чисел в ${ }^{A} S T$. Очевидно, что для самосогласования взаимодействий между пространствамивременами ${ }^{L} S T$ и ${ }^{D} S T$, без применения комплексных чисел в ${ }^{L} S T$ и темных комплексных чисел в ${ }^{D} S T$ нам не обойтись. В разделе 3.5 мы уже кратко упоминали

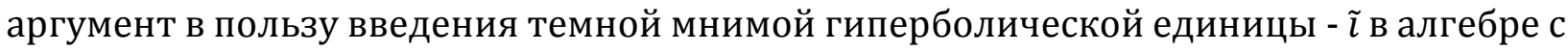
доминантным минусом. Там же мы упоминали о расширенном определении ТФКП. 0 том, что эта теория еще и локальная модель-мост, объединяющая свойства привычной для нас алгебры с абсолютным доминантным плюсом (применяемой в ${ }^{L} S T$ ) и алгебры с доминантным минусом. Одновременно с этим, введение понятия темной мнимой гиперболической единицы в алгебре с абсолютным доминантным минусом позволяет предположить о возможности создать теоретическую модельмост на основе темных комплексных чисел, объединяющую свойства «темной» алгебры и алгебры с абсолютным доминантным плюсом. Если примененная сигнатура [-1] позволило нам выдвинуть гипотезу и получить представление о некоторых свойствах множества темных чисел $(\mathbb{D})$, то примененная сигнатура $\left[-1^{-1}\right]$ предоставила нам также информацию об их геометрическом расположении относительно множества действительных чисел $(\mathbb{R})$. C учетом свойств, хорошо известной функции $y=\frac{1}{x}$ (для рассматриваемого нами случая существенным является инверсия действия значений темных чисел с оси абсцисс $x$ на ось ординат $y$ ) мы можем сделать вывод о том, что темные числа $D a$ действуют так, как будто они располагаются взаимоперпендикулярно относительно оси действительных чисел $R e$. Можно также предположить, что обе теории, ТФКП и будущая теория на основе темной мнимой гиперболической единицы $\tilde{l}$, согласуются с двумя комплексными плоскостями (см. рис. 5), которые должны объединиться на обобщенной Суперкомплексной плоскости (см. рис. 6) и обеспечить самосогласованность во взаимодействиях тканей пространств-времен ${ }^{L} S T$ и ${ }^{D} S T$. 

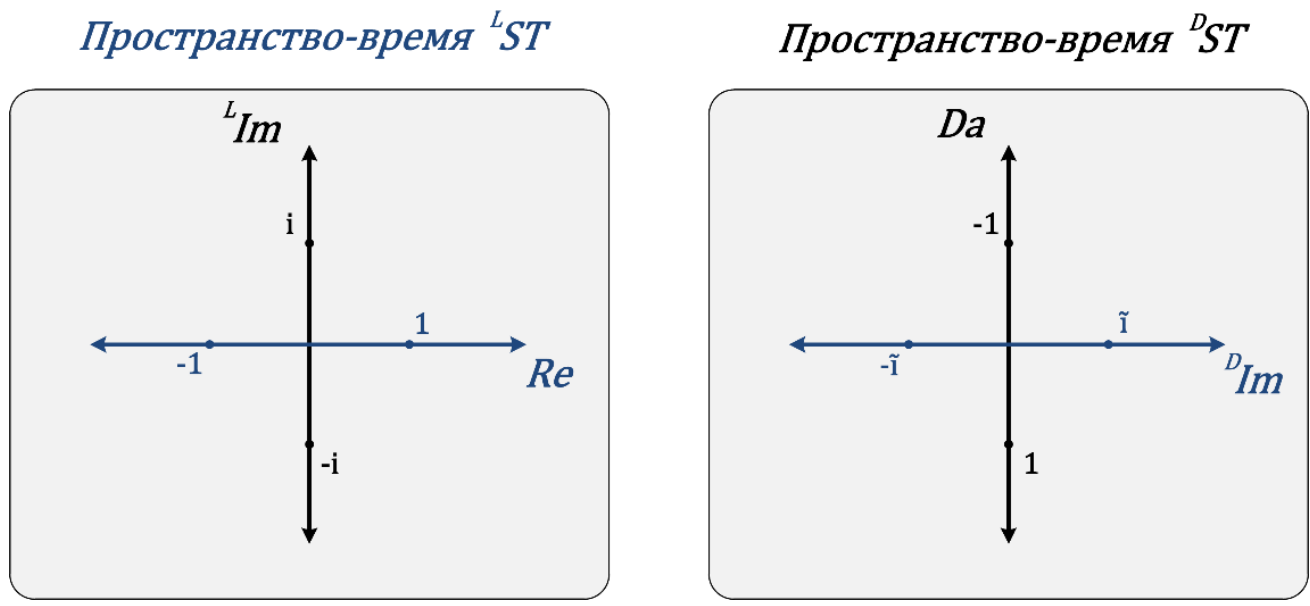

Рис. 5. Комплексная плоскость в ${ }^{L} S T$ и гипотетическая темная комплексная плоскость в ${ }^{\boldsymbol{D}} \boldsymbol{S T}$. В левой части представлена, привычная в математике, комплексная плоскость в ${ }^{L} S T$ с мнимой эллиптической единицей $i$, где $R e-$ ось действительных чисел, а ${ }^{L} I m$ - ось мнимых чисел. В правой части представлена гипотетическая темная комплексная плоскость в ${ }^{D} S T$ с мнимой гиперболической единицей $\tilde{l}$, которая согласно выдвинутой гипотезе, образуется весьма нетривиальным способом (инверсией действия значений темных чисел с оси абсцисс $x$ на ось ординат $y)$, где $D a$ - ось проекции действия темных чисел, a ${ }^{D} I m-$ ось темных мнимых чисел.

Еще одним аргументом в пользу такого развития теории, является то, что скорость света $c$ в ${ }^{L} S T$ для бесконечной Вселенной - является ничтожной величиной. А понятие, усредненной скорости темного света: $-\frac{1}{c}$ (для алгебры с абсолютным доминантным минусом) и его свойства в ${ }^{D} S T$ - еще предстоит изучить (но вряд ли эти свойства изменят отношение автора к этой проблеме). С учетом этих факторов, появление в формулах мгновенного мнимого времени it в ${ }^{L} S T$ и мгновенного темного мнимого времени $\tilde{i} t$ в ${ }^{D} S T$ непреложно обеспечит самосогласованность (в $T_{\text {synch }}$ ) взаимодействий тканей пространств-времен ${ }^{L} S T$ и ${ }^{D} S T$ во всей (в любых областях) бесконечной Вселенной.

Попробуем развить эту идею дальше на основе работ $[70,71,72]$ по обобщению: комплексных чисел в геометрии, кривизны пространства и универсальной алгебры. Мы можем ввести Управляющую Супермнимую Единицу, обозначенную как - ${ }_{s}$, значение квадрата которой будет зависеть от кривизны пространства-времени:

$$
{ }_{S} I^{2}= \begin{cases}\text { для } \Omega=0 \text { в }{ }^{A} S T: I^{2}=\infty \text { и } I^{2}=0 \text { (где, } I-\text { параболическая единица) } \\ \text { для } \omega>0 \text { в }{ }^{L} S T: & i^{2}=-1 \text { (где, } i-\text { эллиптическая единица) } \\ \text { для } \omega<0 \text { в }{ }^{D} S T: & \tilde{\imath}^{2}=+1 \text { (где, } \tilde{\imath}-\text { гиперболическая единица) }\end{cases}
$$

Необходимо сразу же подчеркнуть, что в отличие от исследований, представленных М. А. Лаврентьевым и Б. В. Шабатом в работе [73], где обобщенные комплексные числа вводятся в одном единственном пространстве-времени (естественно, что это ${ }^{L} S T$ ), в нашем случае вопрос введения делителя нуля в темную алгебру с мнимой гиперболической единицей $\tilde{\imath}$, применяемой в ${ }^{D} S T$, - требует отдельного специального рассмотрения (см. столбец 3 Табл. 4). Более того, из соображений корректности (или 
снятия возможных противоречий), мы вынуждены выдвинуть гипотезу о том, что в пространстве-времени ${ }^{D} S T$, в котором, по нашему мнению, применяются темные числа, темные комплексные числа, темные кватернионы и т.д., должна действовать теорема, эквивалентная теореме Фробениуса [74], действующей в пространствевремени ${ }^{L} S T$.

В конечном итоге, оперируя тремя пространствами-времени, мы рассмотрели новый способ задания обобщенных комплексных чисел, в котором квадрат Управляющей Супермнимой Единицы ${ }_{S} I^{2}-$ зависит от кривизны пространства-времени. В момент $B B$ в первичном (материнском) Абсолютном пространстве-времени ${ }^{A} S T$ нулевой $(\Omega=0)$ кривизны (это равенство, а также первая строка выражения 24.0 будет дополнено и уточнено в п. 4.2 Главы 4) реализуется мнимая параболическая единица $I$, квадрат которой может принимать одновременно два значения $\left(I^{2}=\infty\right.$ и $\left.I^{2}=0\right)$. В пространстве-времени положительной кривизны ${ }^{L} S T$ - реализуется мнимая эллиптическая единица $i\left(i^{2}=-1\right)$. В пространстве-времени отрицательной кривизны ${ }^{D} S T$ - реализуется мнимая гиперболическая единица $\tilde{\imath}\left(\tilde{l}^{2}=+1\right)$.

\subsection{0 Мнимая параболическая единица I}

Попробуем понять роль мнимой параболической единицы $I$ в пространстве-времени ${ }^{A} S T$. Мы выдвигаем гипотезу о том, что в первичном (материнском) Абсолютном пространстве-времени ${ }^{A} S T$ в момент $B B$ мнимая параболическая единица - I обладает следующими свойствами: ее квадрат может давать одновременно два значения: $I^{2}=0$ и $I^{2}=\infty$.

Из этой гипотезы следует справедливость следующих соотношений в ${ }^{A} S T$ :

$$
0 \cdot \infty=1 ; 1 / 0=\infty ; 1 / \infty=0 ;
$$

Возможно, к ним следует добавить еще два соотношения:

$$
0 / \infty=1 ; \infty / 0=1 ;
$$

Таким удивительным образом свойства мнимой параболической единицы I позволяет устранить все возможные неопределенности, связанные с этими понятиями и/или числовыми значениями, которые действуют только в Абсолютном пространстве-времени ${ }^{A} S T$ и не распространяются на пространства-времена ${ }^{L} S T$ и ${ }^{D} S T$.

Основной целью и задачей мнимой параболической единицы $I$ - является использование своих свойств для инициации $B B$ путем проведения нескольких синхронных процессов:

- приведение всех базовых параметров к значениям равным мнимой параболической единицы 1;

- начало параболическому расслоению всех базовых параметров, которые троятся, и дополнительно образуют пары: 0 и $+\infty$ или 0 и $-\infty$.

- соответственно, троится Абсолютное пространство-время ${ }^{A} S T$;

- рожденные пространства-времена являются взаимопрозрачными и подразделяются по знаку на: пространство-время положительной кривизны ${ }^{L} S T$ и пространство-время отрицательной кривизны ${ }^{D} S T$; 
- $\quad$ в пространство-время положительной кривизны ${ }^{L} S T$ попадают все $+\infty$ и 0 , если

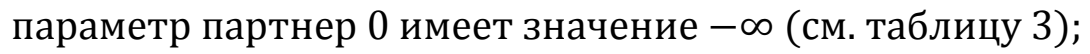

- в пространство-время отрицательной кривизны ${ }^{D} S T$ попадают все $-\infty$ и 0 , если

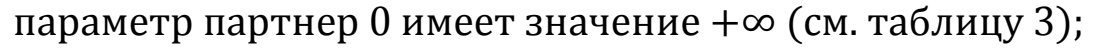

- распределение пар начальных значений базовых параметров в пространствахвремени ${ }^{L} S T$ и ${ }^{D} S T$ создается таким образом, чтобы в обобщенном Паракомплексном ${ }_{I}^{A} S T$ возникал межпространственный потенциал ${ }^{S T} U$ (см. табл. 4).

Таблица 4. Начальные значения базовых параметров в момент $B B$ в трех пространствах-времени ${ }^{A} S T,{ }^{L} S T$ и ${ }^{D} S T$. Данные в столбцах 2 и 3 приведены в соответствии с выбором приоритетности точек отсчета и элементов сигнатуры в ${ }^{L} S T$ и ${ }^{D} S T$ (см. п. 3.7 данной Главы).

\begin{tabular}{|c|c|c|c|c|}
\hline Пространство-время ${ }_{I}^{A} S T$ & \multicolumn{2}{|c|}{ Пространство-время ${ }^{L} S T$} & \multicolumn{2}{|c|}{ Пространство-время ${ }^{D} S T$} \\
\hline & \multicolumn{2}{|c|}{$\begin{array}{c}\text { Приоритет точки } \\
\text { отсчета с элементом } \\
\text { сигнатуры }\left[+1^{+1}\right]\end{array}$} & \multicolumn{2}{|c|}{$\begin{array}{c}\text { Приоритет точки } \\
\text { отсчета с элементом } \\
\text { сигнатуры }\left[-1^{-1}\right]^{* *}\end{array}$} \\
\hline${ }^{A} S=\mathbf{1}(\text { constant })^{*}$ & ${ }^{L} S_{0}=0$ & ${ }^{D} s_{0}=-\infty$ & ${ }^{L} S_{0}=\frac{1}{0}$ & ${ }^{D} S_{0}=-\frac{1}{\infty}$ \\
\hline${ }^{A} \Omega=\mathbf{1}(\text { constant })^{*}$ & ${ }^{L} \omega_{0}=\infty$ & ${ }^{D} \omega_{0}=0$ & ${ }^{L} \omega_{0}=\frac{1}{\infty}$ & ${ }^{D} \omega_{0}=\frac{1}{0}$ \\
\hline${ }^{A} T=1$ (constant $)^{*}$ & ${ }^{L} t_{0}=0$ & ${ }^{D} t_{0}=-\infty$ & ${ }^{L} t_{0}=\frac{1}{0}$ & ${ }^{D} t_{0}=-\frac{1}{\infty}$ \\
\hline${ }^{A} V=\mathbf{1}(\text { constant })^{*}$ & ${ }^{L} v_{0}=\infty$ & ${ }^{D} v_{0}=0$ & ${ }^{L} v_{0}=\frac{1}{\infty}$ & ${ }^{D} v_{0}=\frac{1}{0}$ \\
\hline${ }^{A} G r=1$ (constant) ${ }^{*}$ & ${ }^{L} G r_{0}=0$ & ${ }^{D} G r_{0}=-\infty$ & ${ }^{L} G r_{0}=\frac{1}{0}$ & ${ }^{D} G r_{0}=-\frac{1}{\infty}$ \\
\hline${ }^{A} K=\mathbf{1}(\text { constant })^{*}$ & ${ }^{L} K_{0}=\infty$ & ${ }^{D} K_{0}=0$ & ${ }^{L} K_{0}=\frac{1}{\infty}$ & ${ }^{D} K_{0}=\frac{1}{0}$ \\
\hline
\end{tabular}

* указанные значения, в левом столбце - должны быть выражены Паракомплексными первичными числами, теория о которых в настоящий момент отсутствует.

** для алгебры с абсолютным доминантным минусом с элементом сигнатуры $\left[-1^{-1}\right]$, требуется введение делителя нуля.

- мнимая параболическая единица $I$, также троится, рождая мнимую эллиптическую единицу $i$, квадрат которой: $i^{2}=-1$, действующую в пространстве-времени положительной кривизны ${ }^{L} S T$ и мнимую гиперболическую единицу $\tilde{\imath}$, квадрат которой: $\tilde{l}^{2}=+1$, действующую в пространстве-времени отрицательной кривизны ${ }^{D} S T$.

Как мы уже предполагали ранее, три мнимые единицы образуют обобщенную Паракомплексную плоскость (см. рис. 6), объединяющую комплексную плоскость для ${ }^{L} S T$ и темную комплексную плоскость для ${ }^{D} S T$ и играют, согласно нашей гипотезе главную управляющую роль в согласовании всех взаимодействий в пространствахвремени ${ }^{L} S T$ и ${ }^{D} S T$, и в конечном итоге, создают возможность реализации «эффекта самосогласованности» Вселенной. 


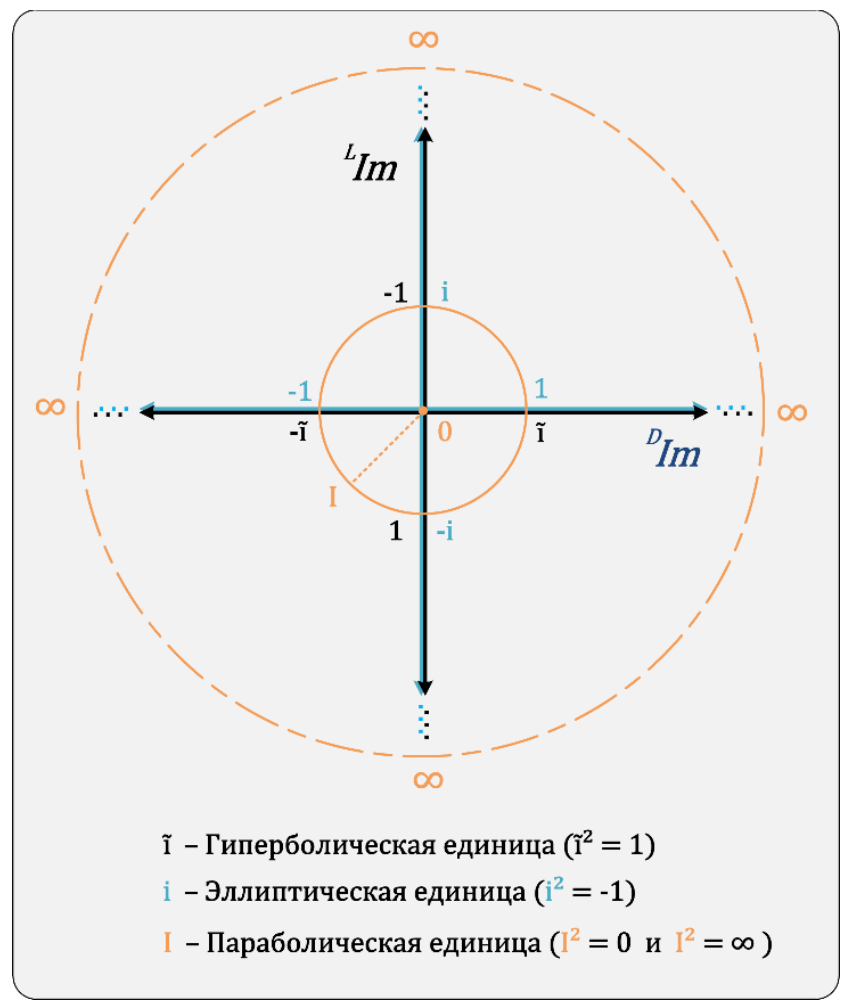

Рис. 6. Обобщенная Паракомплексная плоскость с мнимой параболической единицей I в ${ }^{A} S T$, объединяющая в себе комплексную плоскость с мнимой эллиптической единицей $i$ в ${ }^{L} S T$, темную комплексную плоскость с мнимой гиперболической единицей $\tilde{\boldsymbol{\imath}}$ в ${ }^{D} S T$.

Вывод: Необходимо найти алгоритм и сигнатуру с участием Управляющей Супермнимой Единицы ${ }_{S} I$, с тем, чтобы попытаться вывести формулы расширяющие (комплексифицирующие) уравнения 15 или 23 с участием мнимой параболической единицы $I$ и, соответственно, последующего расслоения этих уравнений на $19 \mathrm{~L}, \mathrm{c}$ участием мнимой эллиптической единицы $i$ и 19D, с участием мнимой гиперболической единицы $\tilde{l}$, до 4 пространственных и 2 временных измерений (для каждого пространства-времени). Такой подход должен обеспечить решение обозначенных в этой главе задач.

\subsection{1 О темных числах}

С учетом полученных в пп. 3.5, 3.7, 3.9 этой Главы результатов, неизбежно возникают два вопроса. Первый, что из себя представляют темные числа $D a$ и множество темных чисел $(\mathbb{D})$ ? Второй, являются ли темные числа точной копией действительных чисел из ${ }^{L} S T$, отличающиеся от них лишь знаком и действующими так, как будто располагаются взаимоперпендикулярно по отношению к ним? Для того, чтобы попытаться ответить на эти вопросы необходимо учесть следующие факторы.

Если исходить из исходных положений Главы 2, из полученной нами обобщенной Паракомплексной плоскости, представленной на рис. 6, из самой сути стационарной самосогласованной Вселенной, то становится очевидным что Вселенная - является математической по своей природе [75]. Из этого утверждения следует весьма важные выводы о том, что само понятие числа - является не абстрактным элементом, а 
важнейшим математическим инструментом необходимым для выполнения возложенных на него задач и функций. Таким образом, множество действительных чисел $(\mathbb{R})$ должно строго соответствовать свойствам пространства-времени ${ }^{L} S T$. Они (действительные числа) должны быть запрограммированы в алгоритме рождения и развития этого пространства-времени и позволять описывать его с момента $B B$ до $C r$. Например, пространство-время ${ }^{L} S T$ - является однородным и изотропным (на больших масштабах), расширяется с момента $B B$ до $C r$. Расстояние в нем - является непрерывным, что соответствует аксиоме о непрерывности множества действительных чисел. Время ${ }^{L} T$ - является однородным и изотропным. В пространстве-времени ${ }^{L} S T$ реализуется Архимедова метрика.

Аналогичным образом, множество темных чисел $(\mathbb{D})$ должно строго соответствовать свойствам пространства-времени ${ }^{D} S T$ и позволять описывать его с момента $B B$ до $C r$. Например, пространство-время ${ }^{D} S T$ - является неоднородным и анизотропным, сжимается с момента $B B$ до $C r$. Время ${ }^{D} T$ - является неоднородным и анизотропным. Определение понятия расстояния в ${ }^{D} S T$, которое может быть разрывным - является предметом для дальнейших исследований. В пространстве-времени ${ }^{D} S T$ реализуется неархимедова метрика.

В соответствии, с предлагаемой теорией оба пространства-времени ${ }^{L} S T$ и ${ }^{D} S T$ вынужденно взаимодействуют друг с другом посредством обобщенного Паракомплексного Абсолютного пространства-времени (см. Главы 6-11). В соответствии с теоремой Островского поле рациональных чисел $\mathbb{Q}$ может быть пополнено двумя эквивалентными путями: либо до поля действительных чисел $\mathbb{R}$, либо до поля р-Адических чисел $\mathbb{Q}_{p}$.

Вполне естественно, что действительные числа $R e$ находит свое применение в пространстве-времени ${ }^{L} S T$, как соответствующие его свойствам. А, вот что касается рАдических чисел, то в соответствии с гипотезой автора, они в гораздо большей степени находят свое применение в пространстве-времени ${ }^{D} S T$. Главной причиной для такого утверждения - является то, что свойства р-Адических чисел больше соответствуют неархимедовым свойствам пространства-времени ${ }^{D} S T$ (см. Главы 511). Предположение о том, что на Планковских масштабах ( $10^{-34}$ см.) - свойства пространства-времени ${ }^{L} S T$ могут кардинально измениться и там находит свое применение р-Адическая квантовая физика - не имеют достаточных оснований. Однако, все эти дискуссии о «объекте» применимости р-Адических чисел и их классификации, практически никак не влияют на ценность уже проведенных исследований в этой области и никоим образом не уменьшают тот огромный вклад, сделанный в развитие р-Адической физики за все эти годы $[76,77]$.

Таким образом, ответом на второй вопрос, заданный в начале этого раздела, будет однозначное нет. Что касается ответа на первый вопрос, то темные числа $D a$ (множество темных чисел $\mathbb{D}$ ) требуют своего дальнейшего глубокого изучения, как собственно говоря и свойства самого пространства-времени ${ }^{D} S T$, в котором они реализуются и которое они должны описывать. 


\subsection{2 Подведение предварительных итогов Главы 3}

В результате наших теоретических исследований, в момент $B B$ первичное (материнское) Абсолютное пространство-время ${ }^{A} S T$ с геометрией Евклида $(\mathcal{E})$, с эталонной метрикой, со статичной тканью пространства и Управляющая Супермнимая Единица ${ }_{s} I$ образуют обобщенное Паракомплексное Абсолютное пространство-время ${ }_{I}^{A} S T$, включающее в себя сферу с базовыми параметрами, равными мнимой параболической единице $I\left(S_{s p h}=I, \Omega_{s p h}=I,{ }^{A} T=I,{ }^{A} V=I\right.$, $\left.{ }^{A} G r=I,{ }^{A} K=I\right)$. Одновременно с этим, в результате частичного параболического расслоения ${ }^{A} S T$ в нем рождаются и динамично развиваются два взаимопрозрачных некоммутативных самосогласованных пространства-времени: ${ }^{L} S T$ и ${ }^{D} S T$ (см. рис. 1 и рис. 4).

Поскольку судьба, образовавшихся в $T_{\text {synch }}$ в обобщенном Паракомплексном Абсолютном пространстве - времени ${ }_{I}^{A} S T$ в момент $B B$ двух пространств-времен ${ }^{L} S T$ и ${ }^{D} S T$ предрешена: в будущем они исчезнут в момент $C r$, также, в $T_{\text {synch }}$, то мы имеем логически-завершенную самосогласованную модель $B B-C r$.

Очевидно, что приведенные примеры алгоритмов полного и частичного параболического расслоения Абсолютного пространства - времени ${ }^{A} S T$ являются предварительными и неполными. Они требуют дальнейшего осмысления и исследований, а единственный и правильный алгоритм - еще предстоит найти.

Вывод: точное определение главных параметров в модели $B B-C r$ (парных и самосогласованных), начальных условий их взаимодействий, верного алгоритма расслоения ${ }^{A} S T$ и самосогласованных формул, определяющих возникновение и дальнейшее развитие пространств-времен ${ }^{L} S T$ и ${ }^{D} S T$ - является основной задачей TSSCU.

Для достижения этой цели рассмотрим подробнее свойства и характеристики каждого из трех пространств-времен: ${ }^{A} S T,{ }^{L} S T$ и ${ }^{D} S T$. 


\section{Глава 4 Свойства и характеристики первичного (материнского) Абсолютного пространства-времени ${ }^{A} S T$}

\section{1 Введение}

Прежде всего необходимо отметить, что существуют объективные и иные разнообразные трудности для того, чтобы исследовать и описывать свойства ${ }^{A} S T$. Приведем некоторые из них. Исследование главного математического объекта, введенного в этой работе Управляющей Супермнимой Единицы ${ }_{s} I-$ находится в самом начале своего пути. Неизвестно, являются ли отношения между ${ }_{s} I$ и ${ }^{A} S T$ независимыми и взаимопрозрачными, как отношения идеалистического и первичного материального начала? Неизвестно также, что заставляет ${ }_{S} I$ инициировать процесс $B B$ и последующего $C r$ в ${ }^{A} S T$ ? И, наконец неизвестно, какими математическими объектами являются: первичные числа, которые мы можем использовать для описания свойств ${ }^{A} S T$, а также Паракомплексные числа в состав которых входит мнимая параболическая единица? Тем не менее, некоторые предварительные предположения и выводы, на уровне гипотезы и интуиции, можно обсудить в этой Главе, чтобы стимулировать дискуссию на эти важнейшие темы.

\section{2 Соответствие каждого 0 и œ. Неразрывность этих понятий и значений друг от друга в пространства-времени ${ }^{A} S T$}

Возникает естественный вопрос, чему могут быть равны базовые параметры в первичном (материнском) ${ }^{A} S T$ ? С учетом того, что в ${ }^{A} S T$ реализуется геометрия Евклида с нулевой ${ }^{A} \Omega=0$ кривизной, можно предположить, что они равны следующим значениям:

$$
{ }^{A} S^{2}=\infty ;{ }^{A} T^{2}=\infty ;{ }^{A} \Omega=0 ;{ }^{A} V=0 ;{ }^{A} G r^{2}=\infty ;{ }^{A} K=0 .
$$

Но тогда возникает второй вопрос, о корректности таких значений в первичном (материнском) Абсолютном пространстве-времени ${ }^{A} S T$ ? Попробуем разобраться в этом вопросе на примере расстояния-радиуса сферы, значение которой предполагается равной ${ }^{A} S^{2}=\infty$. Согласно гипотезе автора, невозможность определения 0, делает предполагаемое значение $\infty$ - некорректным. Это связано с тем, что пара значений: $\infty$ и 0 - являются неразрывными друг от друга в ${ }^{A} S T$. Для каждого 0 должна быть строго своя, соответствующая ему и единственная $\infty$. И, наоборот, у каждой $\infty$ - должен быть строго свой, соответствующей ей и единственный 0 . Эта гипотеза о соответствии и неразрывности каждого нуля 0 и своей бесконечности $\infty$, и наоборот, приводит к тому, что все шесть базовых параметров в ${ }^{A} S T$ имеют одновременно два значения. А. именно:

- расстояние-радиус сферы: ${ }^{A} S^{2}=0$ и ${ }^{A} S^{2}=\infty$;

- время: ${ }^{A} T^{2}=0$ и ${ }^{A} T^{2}=\infty$;

- кривизна: ${ }^{A} \Omega=0$ и ${ }^{A} \Omega=\infty$;

- скорость: ${ }^{A} V=0$ и ${ }^{A} V=\infty$;

- гравитация: ${ }^{A} G r^{2}=0$ и ${ }^{A} G r^{2}=\infty$;

- температура: ${ }^{A} K=0$ и ${ }^{A} K=\infty$. 
Такой подход, только на первый взгляд кажется неожиданным. При более глубоком рассмотрении он становится естественным и закономерным. В соответствии с ним, геометрия Евклида, реализуемая в ${ }^{A} S T$ хорошо согласуется с двумя значениями кривизны ${ }^{A} \Omega=0$ и ${ }^{A} \Omega=\infty$, действующими одновременно. А, выражение 24.0, которое являлось задающим обобщенные комплексные числа необходимо скорректировать и записать следующим образом:

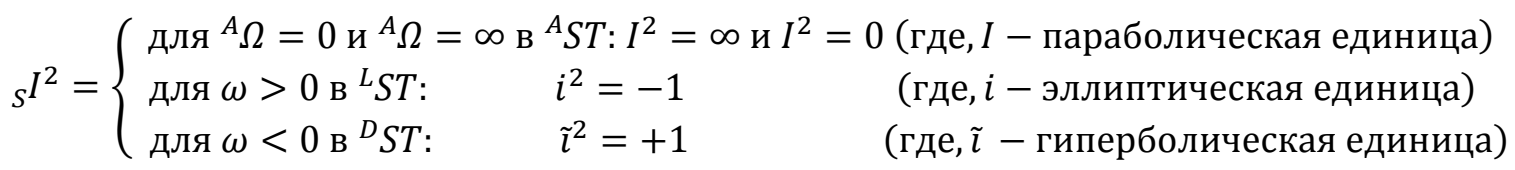

Таким образом, эта гипотеза предоставляет новые возможности в понимании и развитии модели $B B-C r$.

\section{3 Изменение свойств и характеристик пространства-времени ${ }^{A} S T$ в зависимости от периода Релаксации или периода от Большого Взрыва до очередного Схлопывания}

В соответствии, с изложенными в предыдущих Главах 2 и 3 положений, свойства и характеристики первичного (материнского) Абсолютного пространства-времени ${ }^{A} S T$ кардинально меняются в зависимости от двух, сменяющих друг друга периодов, (см. Рис. 7). Первый период начинается, когда Управляющая Супермнимая Единица ${ }_{S} I$ входит во взаимодействие с ${ }^{A} S T$ и возникает очередной Большой Взрыв $\left(B B_{n}\right)$ с последующим Схлопыванием $\left(C r_{n}\right)$ и, так далее. Второй период Релаксации (или $C r_{n}-$ $B B_{n+1}$ и, так далее) возникает в момент очередного $C r$, когда Управляющая Супермнимая Единица ${ }_{S} I$ перестает взаимодействовать с ${ }^{A} S T$.

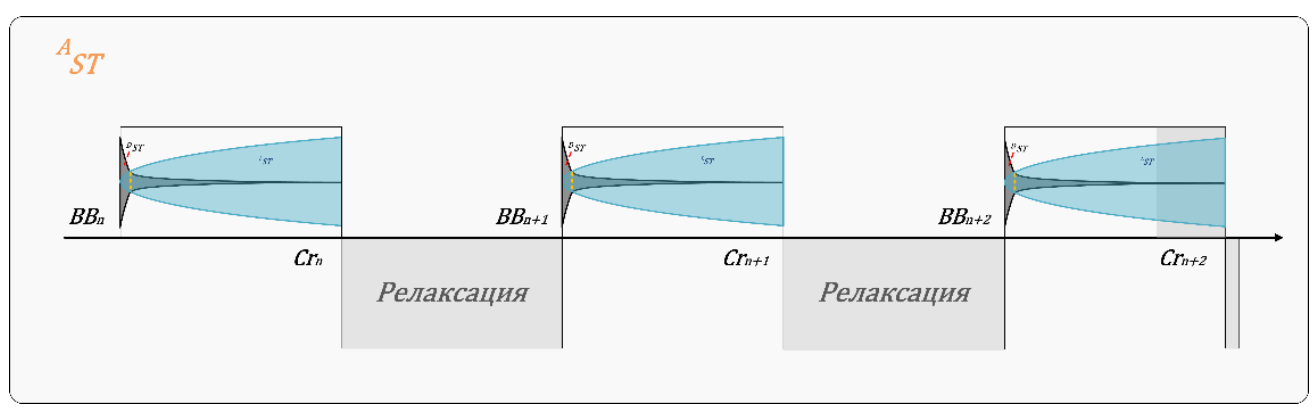

Рис. 7. Периодограмма $\boldsymbol{B B}$ - $\boldsymbol{C} \boldsymbol{r}$ во Вселенной. Первичное (материнское) Абсолютное пространство-время ${ }^{A} S T$ нулевой $(\Omega=0)$ и бесконечной $(\Omega=\infty)$ кривизны одновременно, служит основой для осцилляций Управляющей Супермнимой Единицы ${ }_{S} I$, периодически инициирующей Большой Взрыв $B B$ с последующим Схлопыванием $\mathrm{Cr}$ и периодом релаксации.

\section{4 Период Релаксации $\left(C r_{n-1}\right.$ - $\left.B B_{n}\right)$. Состояние неопределенности базовых параметров}

В момент $C r$, Управляющая Супермнимая Единица ${ }_{S} I$ перестает взаимодействовать с ${ }^{A} S T$. Соответственно, первичное (материнское) Абсолютное пространство-время ${ }^{A} S T$ становится пустым, безжизненным, лишенным каких-либо процессов и единиц измерения, в связи с отсутствием мнимой параболической единицы $I$, задающей соотношения 25 и 26.

На основании вышеизложенного можно предположить, что все базовые параметры в период релаксации пребывают в состоянии неопределенности. 
Вывод: первичное (материнское) Абсолютное пространство-время ${ }^{A} S T$ в период релаксации или периоде $C r_{n-1}-B B_{n}$ - находится в состоянии неопределенности.

\section{5 Период от Большого Взрыва до очередного Схлопывания $\left(B B_{n}-C r_{n}\right)$}

На основании вывода предыдущего параграфа роль Управляющей Супермнимой Единицы ${ }_{S} I$, инициирующей $B B$ - становится более осмысленной. Она вносит в первичное (материнское) Абсолютное пространство-время ${ }^{A} S T$ столь необходимую мнимую параболическую единицу, устанавливая соотношения 25 и 26.

Рассмотрим процессы действия мнимой параболической единицы - I в момент (преддверии) $B B$ на примере уравнения 1 , где $S$ - расстояние-радиус сферы равен:

$$
S^{2}=\left(d x^{2}+d y^{2}+d z^{2}\right)
$$

Правая часть этого уравнения, согласно нашему предположению, в ${ }^{A} S T$ может быть равна минимальному и максимальному корректным значениям в математике: 0 и $+\infty$. Тогда, соответственно, если $S^{2}=0$

$$
S=\sqrt{0}
$$

В момент $B B$, вместо 0, вступает в силу квадрат мнимой параболической единицы - $I^{2}$, и в результате получается:

$$
S=\sqrt{I^{2}}
$$

Далее, соответственно:

$$
S=I=\mathbf{1}
$$

Если $S^{2}=\infty$ :

$$
S=\sqrt{\infty}
$$

В момент $B B$, вместо $\infty$, вступает в силу квадрат мнимой параболической единицы $I^{2}$, и в результате получается:

$$
S=\sqrt{I^{2}}
$$

Далее, соответственно:

$$
S=I=1
$$

Такими удивительными путями, нуждающимися в придирчивой критике мы получаем Управляющую сферу с расстоянием-радиусом равному мнимой параболической единице, которая как в эффекте домино полностью преображает параметры, входящие в уравнение 15 и задает старт параболическому расслоению в ${ }^{A} S T$, а также начало очередного периода $B B-C r$. 
Ниже приводятся некоторые свойства и характеристики ${ }_{I}^{A} S T$.

1. Размерность

В момент $B B$ и до $C r$ первичное (материнское) Абсолютное пространство-время ${ }^{A} S T$ и мнимая параболическая единица $I$ образуют обобщенное Паракомплексное пространство-время ${ }_{I}^{A} S T$, состоящего из 6 паракомплексных измерений:

- 3 - пространственных координат ${ }^{A} S T$;

- 1 - времени ${ }^{A} T$ в ${ }^{A} S T$;

- 1 - мнимой параболической пространственной единицы $I$;

- 1 - мнимого параболического времени ${ }_{I}^{A} T$.

Рассмотрение размерностей самосогласованных пространств-времен ${ }^{L} S T$ и ${ }^{D} S T$ входящих в ${ }_{I}^{A} S T$ не входит в цели данной Главы. Они будут рассмотрены в Главах 5 и 12 данной статьи.

2. Значение базовых параметров в ${ }_{I}^{A} S T::$

- $\quad$ расстояние-радиус сферы: ${ }^{A} S^{2}=0$ и ${ }^{A} S^{2}=\infty$ с параболической мнимой единицей $I$;

- время: ${ }^{A} T^{2}=0$ и ${ }^{A} T^{2}=\infty$ с параболической мнимой единицей $I$;

- кривизна: ${ }^{A} \Omega=0$ и ${ }^{A} \Omega=\infty$ с параболической мнимой единицей $I$;

- скорость: ${ }^{A} V=0$ и ${ }^{A} V=\infty$ с параболической мнимой единицей $I$;

- гравитация: ${ }^{A} G r^{2}=0$ и ${ }^{A} G r^{2}=\infty$ с параболической мнимой единицей $I$;

- температура: ${ }^{A} K=0$ и ${ }^{A} K=\infty$ с параболической мнимой единицей $I$.

3. Базовые категории в ${ }_{I}^{A} S T$ :

- $\quad$ Материя ${ }^{A} M$ : первичная управляющая с параболической мнимой единицей $I$;

- Энергия ${ }^{A} E$ : первичная управляющая с параболической мнимой единицей $I$;

- Информация ${ }^{A} I$ : первичная управляющая с параболической мнимой единицей $I$.

4. Геометрия в ${ }_{I}^{A} S T$ :

- Эвклида + мнимая параболическая сфера с базовыми параметрами равными I;

- П Пятый постулат: в плоскости через точку, не лежащую на данной прямой, можно провести одну и только одну прямую, параллельную данной;

- Сумма углов треугольника: $\angle \alpha+\angle \beta+\angle \gamma=180^{\circ}$.

5. Энтропия

Нахождения подходов для исследования важнейшего понятия энтропии ${ }^{A} H$ в пространстве-времени ${ }^{A} S T$ в обоих периодах: с $C r_{n-1}$ до $B B_{n}$ и с $B B_{n}$ до $C r_{n}-$ является исключительно перспективным и может дать ответы на некоторые вопросы.

Выводы: многие положения, представленные в этой Главе - являются дискуссионными и требуют дальнейших всесторонних исследований. 


\section{Глава 5 Сравнительные свойства и характеристики пространств- времен ${ }^{L} S T$ и ${ }^{D} S T$}

Ниже приводится описание и сравнение антагонистических свойств и характеристик двух пространств-времен ${ }^{L} S T$ и ${ }^{D} S T$.

\section{1 Размерность пространства и времени}

\begin{tabular}{|c|c|}
\hline Пространство-время ${ }^{L} S T$ & Пространство-время ${ }^{D} S T$ \\
\hline $\begin{array}{l}\text { Р Размерность пространства: 4-х } \\
\text { мерное, } 3 \text { пространственных } \\
\text { координаты и одно измерение - } \\
\text { времени }{ }^{L} T ; \\
\text { - Время }{ }^{L} T: \text { объективно, непрерывно, } \\
\text { однородно; } \\
\text { - Направленность (вектор или стрела) } \\
\text { времени: из Прошлого в Будущее; } \\
\text { - Мнимая размерность: 2-х мерное, } 1 \\
\text { мнимая эллиптическая единица } i \text { и } 1 \\
\text { измерение мнимого эллиптического } \\
\text { времени }{ }_{i} t .\end{array}$ & 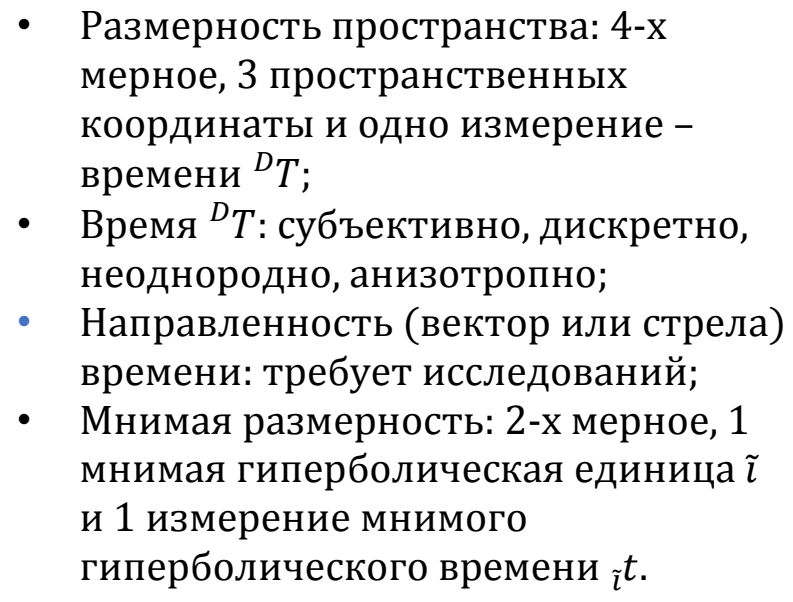 \\
\hline
\end{tabular}

\section{2 Общие закономерности}

\begin{tabular}{|l|l|}
\hline \multicolumn{1}{|c|}{ Пространство-время ${ }^{L} S T$} & \multicolumn{1}{|c|}{ Пространство-время ${ }^{D} S T$} \\
\hline Пространство - однородно и & Пространство- неоднородно и \\
изотропно. & анизотропно. \\
Прямым следствием этого & Имеет дробную размерность, \\
утверждения являются: точные & фрактальную природу [78, 79, 80, 81, \\
фундаментальные константы в ${ }^{L} S T$. & 82, 83] и возможно р-Адическую \\
& структуру [84, 85]. \\
& Прямым следствием этих \\
& характеристик являются: \\
& флуктуационные параметры, \\
& стремящиеся в ${ }^{D} S T$ пределу \\
& значений, являющимися \\
& эквивалентами точным константам \\
& в ${ }^{L} S T$. \\
& \\
\hline
\end{tabular}


5.3 Свойства и характеристики времени ${ }^{L} T$ и времени ${ }^{D} T$

\begin{tabular}{|c|c|}
\hline мя ${ }^{L} S T$ & ство-время ${ }^{D} S T$ \\
\hline 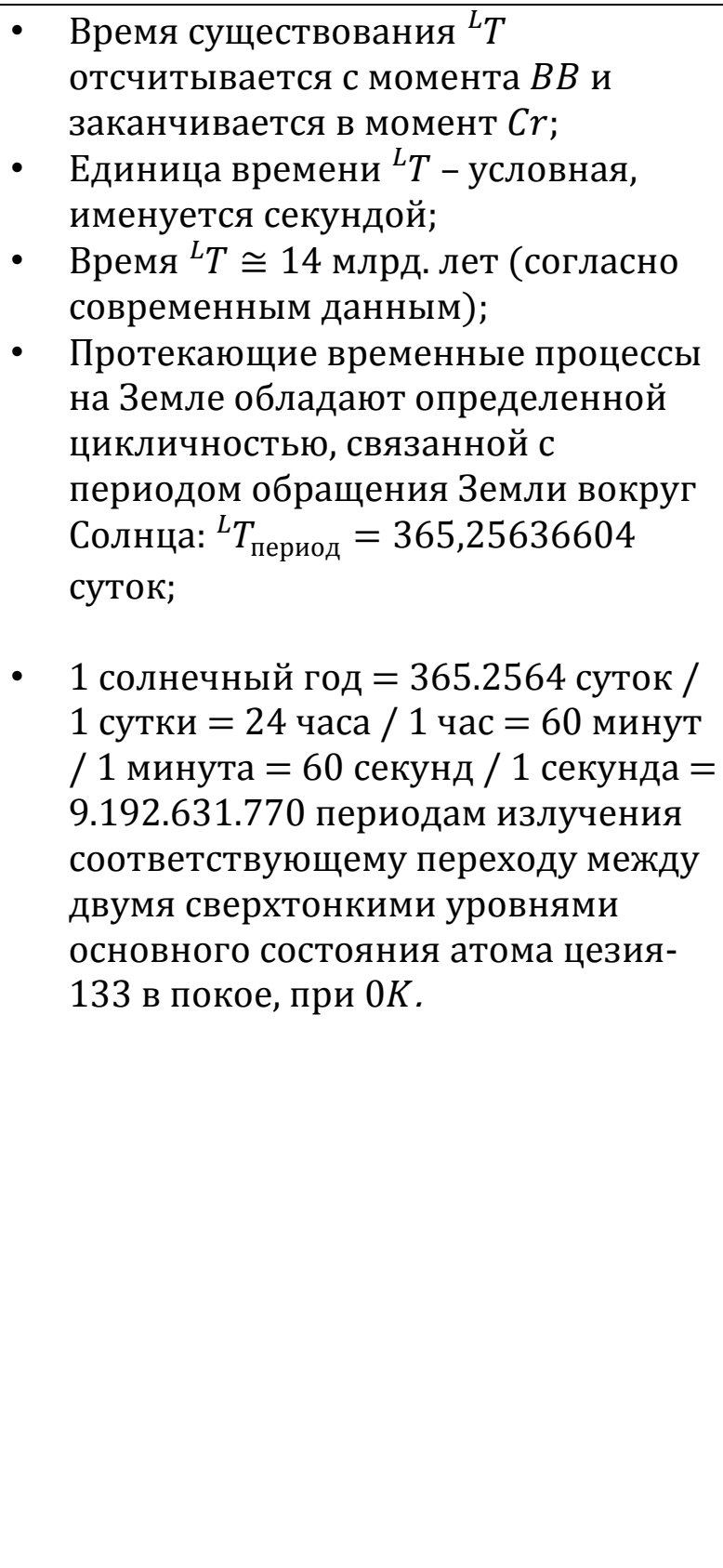 & 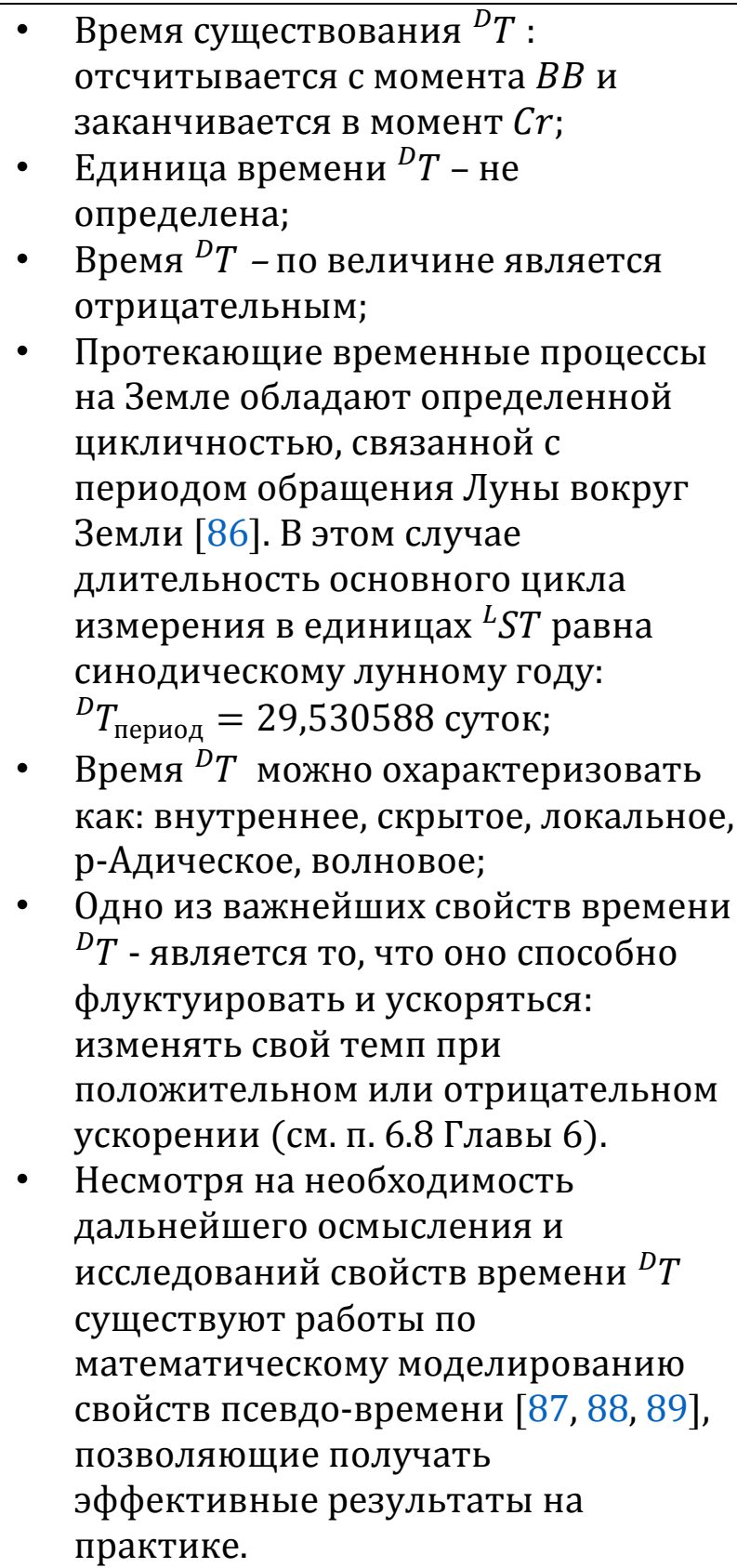 \\
\hline
\end{tabular}


5.4 Кривизна, сумма углов треугольника и свойства

\begin{tabular}{|c|c|}
\hline Пространство-время ${ }^{L} S T$ & Пространство-время ${ }^{D} S T$ \\
\hline $\begin{array}{ll} & \text { Кривизна }{ }^{L} S T \text { положительна } \\
& \left({ }^{L} \omega>0\right) ; \\
\text { - } & \text { Сумма углов треугольника: } \\
& \quad \angle \alpha+\angle \beta+\angle \gamma>180^{\circ} ; \\
\text { - } & \text { Пространство }{ }^{L} S T: \text { расширяется } \\
& \text { однородно относительно }{ }^{A} S T \text { с } \\
& \text { момента } B B \text { до } C r \text { (см. рис. } 1) \text { по } \\
& \text { стреле времени }{ }^{L} T .\end{array}$ & $\begin{array}{ll} & \text { Кривизна }{ }^{D} S T \text { отрицательна } \\
& \left({ }^{D} \omega<0\right) ; \\
\text { - } & \text { Сумма углов треугольника: } \\
& \angle \alpha+\angle \beta+\angle \gamma<180^{\circ} ; \\
\text { - } & \text { Пространство }{ }^{D} S T: \text { сжимается } \\
& \text { неоднородно и анизотропно } \\
\text { относительно }{ }^{A} S T \text { с момента } B B \text { до } \\
C r \text { (см. рис. } 1) .\end{array}$ \\
\hline
\end{tabular}

\section{5 Температура и энтропия}

\begin{tabular}{|c|c|}
\hline Пространство-время ${ }^{L} S T$ & Пространство-время ${ }^{D} S T$ \\
\hline $\begin{array}{l}\text { - } \quad \text { Температура в момент } B B-\text { горячая; } \\
\text { - } \quad \text { Энтропия }{ }^{L} H \text { увеличивается по } \\
\text { стреле времени }{ }^{L} T: \frac{\Delta^{L} H}{\Delta^{L} T}>1 ; \\
\text { - } \quad \text { Энтропия }{ }^{L} H \text { является } \\
\text { отрицательной по отношении к }{ }^{D} H .\end{array}$ & $\begin{array}{l}\text { - } \quad \text { Температура в момент } B B- \\
\text { холодная; } \\
\text { - } \quad \text { Энтропия }{ }^{D} H \text { увеличивается во } \\
\text { времени }{ }^{D} T: \frac{\Delta^{D} H}{\Delta^{D}}<|-1| ; \\
\text { - } \quad \text { Энтропия }{ }^{D} H \text { является } \\
\text { отрицательной по отношению }{ }^{L} H .\end{array}$ \\
\hline
\end{tabular}

\section{6 Геометрия, 5-й постулат и метрика}

\begin{tabular}{|c|c|}
\hline Пространство-время ${ }^{L} S T$ & Пространство-время ${ }^{D} S T$ \\
\hline $\begin{array}{l}\text { • Геометрия Римана }(\mathcal{R}) \\
\text { эллиптическая; } \\
\text { П Пятый постулат: в плоскости через } \\
\text { точку, не лежащую на данной } \\
\text { прямой, нельзя провести ни одну } \\
\text { линию, параллельную этой прямой; } \\
\text { • } \\
\text { Архимедова метрика. } \\
\text { В системе измерений СИ расстояние } \\
\text { измеряется в m (метрах). } \\
\text { Понятие Планковской длины } \\
\left(l_{p} \sim 10^{-34} \text { см) в }{ }^{L} S T \text { - является }\right. \\
\text { предметом дальнейших } \\
\text { исследований. }\end{array}$ & $\begin{array}{ll}\text { • } & \text { Геометрия Лобачевского-Больяи } \\
& (\mathcal{L B}) \text { гиперболическая; } \\
\text { • } & \text { Пятый постулат: в плоскости через } \\
\text { точку, не лежащую на данной } \\
\text { прямой, можно провести } \\
\text { бесконечное количество линий, } \\
\text { параллельных этой прямой; } \\
\text { • } \\
\text { Неархимедова метрика. } \\
\text { Определение расстояния в }{ }^{D} S T- \\
\text { является предметом дальнейших } \\
\text { исследований. }\end{array}$ \\
\hline
\end{tabular}


5.7 Материя, энергия и информация

\begin{tabular}{|c|c|}
\hline Пространство-время ${ }^{L} S T$ & Пространство-время ${ }^{D} S T$ \\
\hline $\begin{array}{ll} & \text { Материя }{ }^{L} M: \text { масса - положительная } \\
& \text { (плотность материи - } \\
& \text { положительная); } \\
\text { • } & \text { Форма материи }{ }^{L} M: \text { частицы; }\end{array}$ & $\begin{array}{ll}\text { - } & \text { Антиматерия }{ }^{D} M: \text { масса - } \\
& \text { отрицательная (плотность } \\
& \text { антиматерии - отрицательная); } \\
\text { - } & \text { Форма антиматерии }{ }^{D} M: \\
& \text { децентрализованная (требует } \\
& \text { исследований); } \\
\text { - } \text { Энергия: }{ }^{D} E-\text { отрицательная, } \\
\text { (плотность энергии - } \\
\text { отрицательная); } \\
\text { - Информация: }{ }^{D} I-\text { фрактальная. }\end{array}$ \\
\hline
\end{tabular}

\section{8 Элементарный электрический заряд $q$ и элементарный топологический магнитный заряд $\tilde{\theta}$}

\begin{tabular}{|c|c|}
\hline Про & Простр \\
\hline 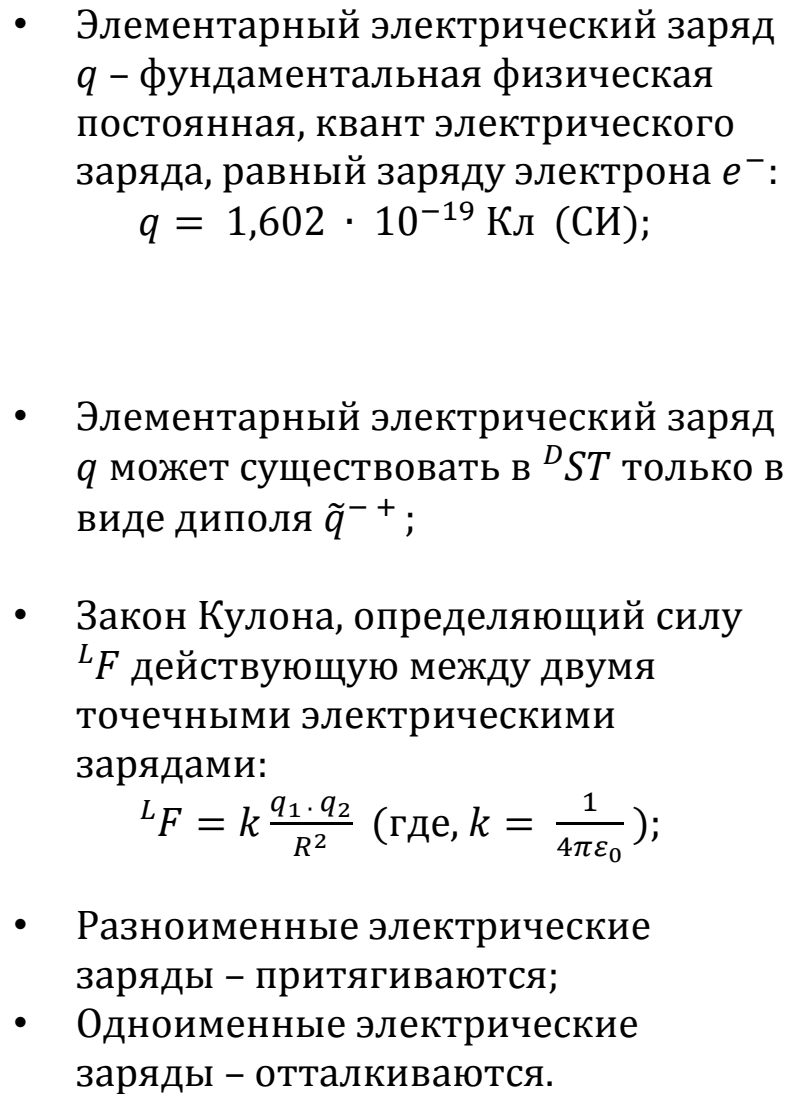 & $\begin{array}{l}\text { • } \text { Элементарный топологический } \\
\text { магнитный заряд } \tilde{\theta}[90,91,92,93]- \\
\text { фундаментальный физический } \\
\text { флуктуационный параметр, квант } \\
\text { магнитного заряда (монополь), } \\
\text { равный заряду позитрона } \tilde{e}^{+}{ }^{D} S T . \\
\text { Численное значение } \tilde{\theta} \text { требует } \\
\text { определения. } \\
\text { • } \text { Элементарный топологический } \\
\text { магнитный заряд } \tilde{\theta} \text { может } \\
\text { существовать в }{ }^{L} S T \text { только в виде } \\
\text { диполя } \theta^{+-} ; \\
\text {Требуется найти Закон(ы), } \\
\text { определяющий(ие) силы }{ }^{D} F_{1-n} \\
\text { действующие между двумя } \\
\text { топологическими магнитными } \\
\text { зарядами } \tilde{\theta}_{1} \text { и } \tilde{\theta}_{2}, \text { эквивалентный(е) } \\
\text { Закону Кулона в }{ }^{L} S T ; \\
\text { Разноименные топологические } \\
\text { магнитные заряды - отталкиваются; } \\
\text { Одноименные топологические } \\
\text { магнитные заряды - притягиваются. }\end{array}$ \\
\hline
\end{tabular}


5.9 Электромагнитные волны (ЭМВ) и позитронно-электрические волны (ПЭВ)

\begin{tabular}{|c|c|}
\hline Пространство-время ${ }^{L} S T$ & Пространство-время ${ }^{D} S T$ \\
\hline $\begin{array}{c}\text { Электромагнитные волны (ЭМВ) с } \\
\text { первичным элементарным } \\
\text { электрическим зарядом и вторичным } \\
\text { магнитным зарядом } \\
\text { (электромагнитное взаимодействие) }\end{array}$ & $\begin{array}{c}\text { Позитронноэлектрические волны (ПЭВ) } \\
\text { с первичным элементарным } \\
\text { топологический магнитным зарядом и } \\
\text { вторичным электрическим зарядом } \\
\text { (сильное взаимодействие) }\end{array}$ \\
\hline 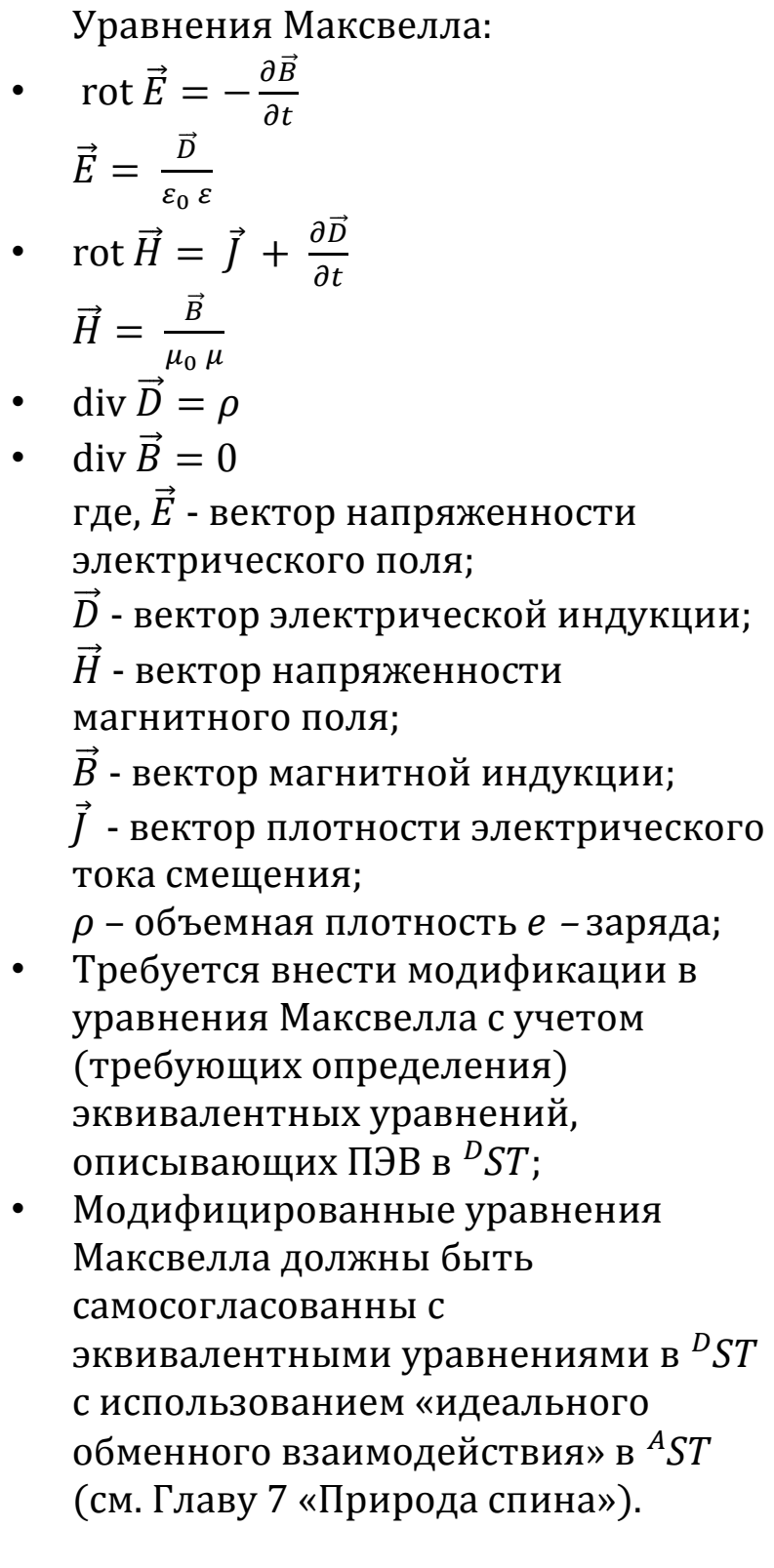 & $\begin{array}{l}\text { - } \text { Требуется найти уравнения, } \\
\text { описывающие позитронно- } \\
\text { электрические волны в }{ }^{D} S T \\
\text { эквивалентные и } \\
\text { самосогласованные с } \\
\text { модифицированными уравнениями } \\
\text { Максвелла в }{ }^{L} S T ; \\
\text { Трудности: } \\
\text { свойства }{ }^{D} S T \text { недостаточно } \\
\text { изучены; } \\
\text { время - }{ }^{D} T \text { - не определено; } \\
\text { подходы к измерению времени - } \\
\text { DT - отсутствуют; } \\
\text { децентрализованная форма материи } \\
\text { в }{ }^{D} S T \text { не определена; } \\
\text { значение элементарного } \\
\text { топологического магнитного заряда } \\
\tilde{\theta} \text { - не установлено; } \\
\text { плотность распределения } \\
\text { элементарного топологического } \\
\text { магнитного заряда } \tilde{\theta} \text { на } \\
\text { децентрализованном объекте } \\
\text { антиматерии в }{ }^{D} S T \text { не известна; } \\
\text { Уравнения, описывающие ПЭВ } \\
\text { должны быть самосогласованны с } \\
\text { эквивалентными } \\
\text { модифицированными уравнениями } \\
\text { Максвелла в }{ }^{L} S T \text { с использованием } \\
\text { «идеального обменного } \\
\text { взаимодействия»в }{ }^{A} S T \text { (см. Главу } 7 \\
\text { «Природа спина»). }\end{array}$ \\
\hline
\end{tabular}


5.10 Фотоны $\gamma$ и темные фотоны $\tilde{\gamma}$

\begin{tabular}{|c|c|}
\hline Пространство-время ${ }^{L} S T$ & Пространство-время ${ }^{D} S T$ \\
\hline $\begin{array}{l}\text { - Форма фотона: частицы материи; } \\
\text { - Масса фотона: } m=0 \text {; } \\
\text { - } \quad \text { Электрический заряд: } q=0 \text {; } \\
\text { - Спин: }{ }_{t} S= \pm 1 .\end{array}$ & $\begin{array}{l}\text { - } \text { Темный свет состоит из темных } \\
\left.\text { фотонов - }{ }^{D} \gamma \text { (или просто } \tilde{\gamma}\right) \\
\text { переносчиков темных квантов }{ }^{D} \hbar \\
\text { темной энергии }{ }^{D} E \\
\text { Позитронноэлектрических Волн } \\
\text { (сильного взаимодействия); } \\
\text { В TSSCU глюоны - } g \text { и магноны - } \\
\text { являются темными фотонами - } \tilde{\gamma} \text {; } \\
\text { Форма темного фотона: } \\
\text { децентрализованные объекты } \\
\text { антиматерии (см. Главу 7); } \\
\text { - Масса темного фотона: } m=0 ; \\
\text { - Магнитный заряд: } \tilde{\theta}=0 ; \\
\text { - Спин: }{ }_{t} S= \pm 1 .\end{array}$ \\
\hline
\end{tabular}

\subsection{1 Скорость света с и темного света $\tilde{c}$}

\begin{tabular}{|c|c|}
\hline Пространство-время ${ }^{L} S T$ & Пространство-время ${ }^{D} S T$ \\
\hline $\begin{array}{l}\text { - Скорость света в вакууме - } c \text {, } \\
\text { состоящего из частиц фотонов } \gamma \text { - не } \\
\text { может превышать: } \\
c=299792458 \text { м/с }\end{array}$ & $\begin{array}{l}\text { - Скорость темного света - } \tilde{c} \text {, } \\
\text { состоящего из темных фотонов } \widetilde{\gamma} \text { - } \\
\text { требует исследований, с учетом } \\
\text { свойств }{ }^{D} S T, \text { метрики задающей } \\
\text { расстояние }-{ }^{D} S, \text { времени }-{ }^{D} T, \text { а } \\
\text { также подходы к определению } \\
\text { единицы измерения - }{ }^{D} T . \\
\text { В алгебре с абсолютным } \\
\text { доминантным минусом она } \\
\text { соответствует усредненному } \\
\text { значению: } \\
\qquad \tilde{c}=-\frac{1}{c}\end{array}$ \\
\hline
\end{tabular}




\section{Глава 6 Трех пространственно-временная модель квантовой положительной гравитации в ${ }^{L} S T$ и многоквантовой отрицательной гравитации в ${ }^{D} S T$}

6.1 Законы всемирного тяготения в ${ }^{L} S T$ и ее эквивалент (антитяготения) в ${ }^{D} S T$

\begin{tabular}{|c|c|}
\hline Пространство-время ${ }^{L} S T$ & Пространство-время ${ }^{D} S T$ \\
\hline $\begin{array}{l}\text { - Закон всемирного тяготения (ЗВТ): } \\
\quad{ }^{L} F=-{ }^{L} G \frac{m_{1} \cdot m_{2}}{R^{2}} \\
\text { где, гравитационная постоянная } \\
{ }^{L} G-\text { отрицательна } \\
{ }^{L} G=-6,67408 \cdot 10^{-11} \mathrm{~m}^{3} \kappa^{-1} \mathrm{c}^{-2} \\
\text { - Все тела притягиваются. }\end{array}$ & $\begin{array}{l}\text { - Требуется найти уравнения для } \\
\text { Закона(ов) антитяготения } \\
\text { эквивалентные уравнению, } \\
\text { описывающему (ЗВТ) в }{ }^{L} S T: \\
\qquad{ }^{D} F_{1-n}=(?) \\
\text { - Все тела отталкиваются. }\end{array}$ \\
\hline
\end{tabular}

6.2 Общая теория относительности (ОТО) в ${ }^{L} S T$ и ее эквивалент в ${ }^{D} S T$

\begin{tabular}{|c|c|}
\hline Пространство-время ${ }^{L} S T$ & Пространство-время ${ }^{D} S T$ \\
\hline $\begin{array}{l}\text { ОТО: } \\
G_{\mu v}+\Lambda_{g_{\mu v}}=\frac{8 \pi \cdot g}{c^{4}} T_{\mu v} \\
\text { где, } G_{\mu v}-\text { тензор Эйнштейна; } \\
\Lambda_{g_{\mu v}}-\text { космологическая постоянная } \\
{ }^{L} g-\text { гравитационная постоянная; } \\
c \text { - скорость света в вакууме; } \\
T_{\mu v} \text { - тензор энергии-импульса } \\
\text { материи }{ }^{L} M ; \\
G_{\mu v}=R_{\mu v}-\frac{1}{2} g_{\mu v} R \\
\text { где, } R_{\mu v}-\text { тензор Риччи; } \\
R-\text { скалярная кривизна; } \\
g_{\mu v}-\text { метрика; }\end{array}$ & $\begin{array}{l}\text { • Требуется найти систему уравнений, } \\
\text { удовлетворяющих условиям } \\
\text { сформулированными в конце п. } 6.7 \\
\text { данной Главы. }\end{array}$ \\
\hline $\begin{array}{l}\text { - ОТО в } T S S C U: \\
\text { 1) } \Lambda_{g_{\mu v}}=0, \\
\text { 2) При отсутствии материи }{ }^{L} M \\
\text { пространство }{ }^{L} S T \text { - не является } \\
\text { пустым, а состоит из ткани, } \\
\text { расширяющейся во времени }{ }^{L} T, \\
R_{\mu v} \neq 0, R \neq 0 \text {, а система уравнений } \\
\text { нуждается в дополнении, } \\
\text { учитывающим фоновое изменение } \\
\text { кривизны }{ }^{L} \omega \text { и расширение тканей } \\
\text { пространства-времени (см. } \\
\text { требования, сформулированные в } \\
\text { конце п. } 6.7 \text { данной Главы). }\end{array}$ & \\
\hline
\end{tabular}


6.3 Постоянная Хаббла ${ }^{L} H_{b}$ в ${ }^{L} S T$ и его эквивалент ${ }^{D} H_{b_{1-n}}$ в ${ }^{D} S T$

\begin{tabular}{|c|c|}
\hline Пространство-время ${ }^{L} S T$ & Пространство-время ${ }^{D} S T$ \\
\hline $\begin{array}{l}\text { - На основе современных научных } \\
\text { данных }[94,95,96,97] \text { нам сегодня } \\
\text { известна «динамическая } \\
\text { постоянная» Хаббла в }{ }^{L} S T \text {, примерно } \\
\text { равная: } \\
\quad{ }^{L} H_{b} \cong 67-74(\mathrm{Kм} / \mathrm{c}) / \text { Мпс }\end{array}$ & $\begin{array}{l}\text { - В пространстве-времени }{ }^{D} S T \\
\text { должны существовать свои, } \\
\text { «динамические флуктуационные } \\
\text { параметры» }{ }^{D} H_{b_{1-n}} \text {, эквивалентные } \\
{ }^{L} H_{b}: \\
\quad{ }^{D} H_{b_{1-n}}=(?)\end{array}$ \\
\hline
\end{tabular}

Таким образом, самосогласованные параметры, которыми оперирует TSSCU, открывает перед нами новые возможности для построения точных математических моделей.

\section{4 Ткани пространств-времен ${ }^{A} S T,{ }^{L} S T$ и ${ }^{D} S T$ - как реальные и объективные составляющие устройства Вселенной}

Основной задачей современной физики - является объединение квантовой физики и ОТО, как лучшей теории гравитации на сегодняшний день. Для этого делаются различные попытки разработать математические модели квантования гравитации или квантового пространства-времени сталкивающиеся с непреодолимыми трудностями [98, 99]. При этом точное определение понятия квантового пространства-времени отсутствует. Обычно, для его описания подразумевают возникновение дискретности в пространстве на Планковских масштабах длины ( $10^{-34}$ см.). В связи с этим, мы можем однозначно заявить, что в одном 6-мерном (4 вещественных: 3 пространственных и 1 временное измерения +2 мнимых: 1 пространственное и 1 временное измерения) пространстве-времени создать свойство квантовости - невозможно. В соответствии с нашей гипотезой, необходимо добавить, как минимум еще одно противодействующее и независимое 6-мерное (с эквивалентными 6 измерениями) пространство-время. При таком взаимодополнении, должны соблюдаться некоторые обязательные условия: взаимопрозрачность обоих пространств-времен, их разнородность и некоммутативность, антагонизм, а также необходимость постоянного взаимодействия друг с другом. Только при строгом соблюдении этих требований, в обоих пространствах-времени возникает свойство квантовости. При этом у каждого из этих двух независимых и антагонистических пространств-времен - будут свои кванты действия, как вынужденный компромисс в постоянной борьбе друг с другом. Добавив, к этим двум независимым пространствам-времени, обобщенное Паракомплексное Абсолютное пространство-время, через которое происходят все взаимодействия и которое является индифферентным (беспристрастным) Арбитром, мы в конечном итоге и получим исходные положения, описанные в Главе 2. Главный вывод к которому мы приходим заключается в том, чтобы еще раз подчеркнуть необходимость и адекватность названия п. 6.4 и нижеследующего утверждения.

С учетом того, что в предложенной нами теории три пространства-времени активно взаимодействуют между собою по заданным алгоритмам, мы вынуждены ввести понятие тканей пространств-времен ${ }^{L} S T$ и ${ }^{D} S T$ как особые-полевые формы материи и антиматерии, содержащих в себе соответствующие энергию и информацию. 
Ткань однородного и изотропного пространства-времени ${ }^{L} S T \quad$ расширяется (относительно неподвижной ткани ${ }^{A} S T$ ) за счет сжатия ткани неоднородного и анизотропного пространства-времени ${ }^{D} S T$. Справедливо и обратное, ткань неоднородного и анизотропного пространства-времени ${ }^{D} S T \quad$ сжимается (относительно неподвижной ткани ${ }^{A} S T$ ) за счет расширения ткани однородного и изотропного пространства-времени ${ }^{L} S T$.

Рассмотрим простейший абстрактный пример-модель плоских элементарных ячеек тканей пространств - времен ${ }^{L} S T$ и ${ }^{D} S T$ в абстрактный момент времени периода $B B$ $\mathrm{Cr}$, изначально образующихся из равностороннего треугольника в ${ }^{A} S T$, см. рис. 8.

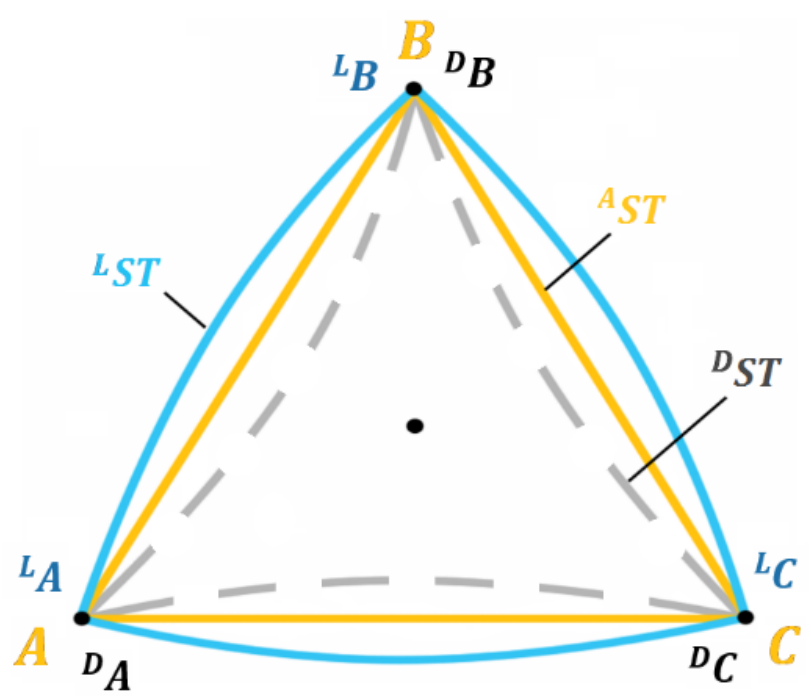

Рис. 8. Абстрактный пример-модель плоских элементарных ячеек пространств-времен ${ }^{L} S T$ и ${ }^{D} S T$, изначально образующихся из элементарной ячейки (равностороннего треугольника) в ${ }^{A} \boldsymbol{S T}$. Точки $A, B$ и $C$ - являются точками Абсолютного пространствавремени ${ }^{A} S T$ в котором синхронно в $T_{\text {synch }}$ рождаются точки ${ }^{L} A$ и ${ }^{D} A,{ }^{L} B$ и ${ }^{D} B,{ }^{L} C$ и ${ }^{D} C$, образуя во времени ${ }^{L} T$ элементарные ячейки пространства-времени положительной кривизны ${ }^{L} S T$ и во времени ${ }^{D} T$ пространства-времени отрицательной кривизны ${ }^{D} S T$, соответственно. Точки ${ }^{L} A$ и ${ }^{D} A,{ }^{L} B$ и ${ }^{D} B,{ }^{L} C$ и ${ }^{D} C-$ являются фундаментально- сцепленными. Для наглядности ячейка в ${ }^{D} S T$ изображена в виде прерывистой кривой, но необходимо сразу оговориться, что форма тканей и антиматерии в ${ }^{D} S T$ требуют отдельного изучения (см. п. 6.8 данной Главы).

Длину геодезической линии в ${ }^{A} S T$ между точками $A$ и $B$ - обозначим через ${ }^{A} S_{g l}$. Длину геодезической линии в ${ }^{L} S T$ между точками ${ }^{L} A$ и ${ }^{L} B$ - обозначим через ${ }^{L} S_{g l}$. 
Обобщенную проекцию на ${ }^{A} S T$ (см. пп. 6.7, 6.8) геодезических линий в ${ }^{D} S T$ между точками ${ }^{D} A$ и ${ }^{D} B-$, обозначим через ${ }^{D} S_{g l}$.

Очевидно, что для ${ }^{A} S T$ с геометрией Евклида $(\mathcal{E})$ геодезическая линия, соединяющая точки $A$ и $B$ и прямая линия (отрезок), соединяющий точки $A$ и $B$ совпадают:

$$
\frac{S_{g l}}{{ }^{A} S_{g l}}=1
$$

Для ${ }^{L} S T$ с геометрией Римана $(\mathcal{R})$ геодезическая линия, соединяющая точки ${ }^{L} A$ и ${ }^{L} B$ всегда больше (длиннее) геодезической линии, соединяющей точки $A$ и $B$ в ${ }^{A} S T$ :

$$
\frac{{ }^{L} S_{g l}}{{ }^{A} S_{g l}}>1
$$

Значение соотношения 34 мы можем назвать коэффициентом геодезической кривизны между точками ${ }^{L} A$ и ${ }^{L} B$ в ${ }^{L} S T$.

Для ${ }^{D} S T$ с геометрией Лобачевского-Больяи $(\mathcal{L B})$ обобщенная проекция геодезических линий, соединяющих точки ${ }^{D} A$ и ${ }^{D} B$ на ${ }^{A} S T$ всегда меньше (короче) геодезической линии, соединяющей точки $A$ и $B$ в ${ }^{A} S T$ :

$$
\frac{\left|{ }^{D} S_{g l}\right|}{{ }^{A} S_{g l}}<1
$$

Значение соотношения 35 мы можем назвать коэффициентом геодезической кривизны между точками ${ }^{D} A$ и ${ }^{D} B$ в ${ }^{D} S T$.

В соответствии с формулами 33-35 между длинами ${ }^{L} S_{g l}$ в ${ }^{L} S T,{ }^{A} S_{g l}$ в ${ }^{A} S T$ и ${ }^{D} S_{g l}$ в ${ }^{D} S T$ выполняется неравенство 36:

$$
{ }^{L} S_{g l}>{ }^{A} S_{g l}>\left|{ }^{D} S_{g l}\right|
$$

Приведенный абстрактный пример-модель строения элементарных ячеек пространств-времен ${ }^{L} S T$ и ${ }^{D} S T$ - является наипростейшим, но автору было важно продемонстрировать, что все элементы тканей обоих пространств-времен - являются фундаментально-сцепленными. Физическая реальность, несоизмеримо сложнее: см. пп. 6.6 - 6.8 настоящей Главы, а также Главу 7. Но в конечном итоге становится очевидным, что для построения полноценной теории гравитации и антигравитации нам необходимо развивать теорию физики строения и взаимодействий тканей пространств-времен ${ }^{L} S T$ и ${ }^{D} S T$.

\section{5 Образование масс ${ }^{L} m$ частиц материи в ${ }^{L} S T$ и масс ${ }^{D} m$ децентрализованных объектов антиматерии в ${ }^{D} S T$}

Гипотеза: массы ${ }^{L} m$ частиц материи ${ }^{L} M$ в ${ }^{L} S T$ и массы ${ }^{D} m$ децентрализованных объектов антиматерии ${ }^{D} M$ в ${ }^{D} S T$ рождаются синхронно в $T_{\text {synch }}$ из фундаментальносцепленных ячеек тканей пространств-времен ${ }^{L} S T$ и ${ }^{D} S T$ с использованием топологических эффектов перепутывания тканей обоих пространств в ${ }^{A} S T$. Эти топологические механизмы - требуют глубоких исследований на основе физических теорий кос и узлов. При этом, образованные геометрические дефекты или 
деформационные-искривления в самих тканях обоих пространств - являются ничтожными и компенсируются. А, вот при движении масс ${ }^{L} m$ частиц материи в ${ }^{L} S T$ и масс ${ }^{D} m$ децентрализованных объектов антиматерии в ${ }^{D} S T$, эти геометрические дефекты, имеющие полевую природу - является их неотъемлемой частью. Эти утверждения хорошо согласуется с ОТО для пространства-времени ${ }^{L} S T$. Рожденные синхронно в $T_{\text {synch }}$ массы ${ }^{l} m$ частиц материи ${ }^{L} M \quad$ в ${ }^{L} S T$ и массы ${ }^{D} m$ децентрализованных объектов антиматерии ${ }^{D} M$ в ${ }^{D} S T$ - также являются фундаментально-сцепленными. Идеи и гипотезы синхронного рождения из вакуума материи с положительной массой и с отрицательной массой периодически изучались в научной литературе [100], но в данном случае постулируется рождение частиц материи и децентрализованных объектов антиматерии непосредственно из взаимопрозрачных тканей пространств-времен ${ }^{L} S T$ и ${ }^{D} S T$, причем каждая частица материи и децентрализованный объект антиматерии - рождаются в своем пространстве-времени.

Исходя из предложенной нами гипотезе, любая масса ${ }^{L} m$ рожденная в ${ }^{L} S T-$ является гравитационной по определению, а любая масса ${ }^{D} m$ рожденная в ${ }^{D} S T$ - является антигравитационной. Следовательно, в стационарном состоянии масса ${ }^{L} m$ в ${ }^{L} S T$ стремиться к покою, а масса ${ }^{D} m$ в ${ }^{D} S T$ стремиться к динамике.

Важнейший и принципиальный вопрос о том, каким образом в ${ }^{L} S T$ появляются частицы антиматерии, проникающие из ${ }^{D} S T$ кратко рассмотрен в Главе 9.

Таким образом, фундаментально-сцепленные между собою ячейки тканей пространств-времен ${ }^{L} S T$ и ${ }^{D} S T$ - являются активными полевыми базисными материальными субстанциями, требующие глубокого изучения.

Исключением из предложенного механизма синхронного в $T_{\text {synch }}$ образования масс ${ }^{L} m$ в ${ }^{L} S T$ и ${ }^{D} m$ в ${ }^{D} S T$ - является: бозон Хиггса H. O скалярном поле Браута-ЭнглераХиггса $B E H$ и самом бозоне $H$, имеющего спин равный $0\left({ }_{t} S=0\right)$, их роли в слабом взаимодействии будет изложено в следующей публикации «Формализм TSSCU».

\section{6 Самосогласованные уравнения рождения и эволюции тканей пространств- времен в условиях гравитации в ${ }^{L} S T$ и антигравитации в ${ }^{D} S T$}

Гравитация в ${ }^{L} S T$ и антигравитация в ${ }^{D} S T$ представляют собою силы, которые возникают как противодействующие (стремящиеся компенсировать) неуклонному расширению пространства-времени ${ }^{L} S T$ и неуклонному сжатию пространствавремени ${ }^{D} S T$. Как было указано в Главе 3, с момента $B B$ одновременное изменение пары обратно квадратично зависимых параметров: расстояния-радиуса кривизны ${ }^{L} S$ в сторону увеличения и кривизны - ${ }^{L} \omega$ в сторону уменьшения - приводит к расширению пространства-времени ${ }^{L} S T$ и порождает гравитацию - ${ }^{L} G_{r}$. Начальные значения этих параметров известны: ${ }^{L} s_{0}=0$, а ${ }^{L} \omega_{0}=\infty$. Синхронные изменения этих параметров должны приводить к созданию непрерывных ориентированных сетей «эллиптического типа» с линейным однородным расширением пространствавремени ${ }^{L} S T$. C момента $B B$ одновременное изменение пары обратно квадратично зависимых параметров: расстояния-радиуса кривизны - ${ }^{D} S$ в сторону уменьшения и 
кривизны - ${ }^{D} \omega$ в сторону увеличения - приводит к неоднородному и анизотропному сжатию пространства-времени ${ }^{D} S T$ и порождает антигравитацию - ${ }^{D} G_{r}$. Начальные значения этих параметров известны: ${ }^{D} S_{0}=-\frac{1}{\infty}$, a ${ }^{D} \omega_{0}=0$. С учетом того, что процесс сжатия - является неоднородным и анизотропным, синхронные изменения этих параметров должны приводить к созданию разрывных вращательных сетей «гиперболического типа» и создавать топологический вращательный механизм стабилизации (см. п. 6.7).

\begin{tabular}{|c|c|}
\hline Пространство-время ${ }^{L} S T$ & Пространство-время ${ }^{D} S T$ \\
\hline$G_{r}=\frac{K \cdot \omega \cdot \sqrt{d x^{2}+d y^{2}+d z^{2}}}{t \cdot v}$ & $-\frac{1}{G_{r}}=\frac{-\frac{1}{K} \cdot-\frac{1}{\omega} \cdot \frac{-1}{\sqrt{-d x^{2}-d y^{2}-d z^{2}}}}{-\frac{1}{t} \cdot-\frac{1}{v}}$ \\
\hline
\end{tabular}

Уравнения 19L и 19D полученные в Главе 3 - являются предварительными и неполными см. п. 3.7 Главы 3. Несмотря на это, они дают нам богатую информацию для анализа и построения различных рабочих моделей гравитации в ${ }^{L} S T$ и антигравитации в ${ }^{D} S T$, а также для проведения практических экспериментов. Необходимо напомнить, что уравнение 19D относится к алгебре с абсолютным доминантным минусом. Главной задачей, стоящей на нынешнем этапе - является нахождения кванта расширения пространства-времени ${ }^{L} S T$ самосогласованного с темными квантами сжатия пространства-времени ${ }^{D} S T$ во времени синхронизации $T_{\text {synch }}$.

6.7 Классификация и новый содержательный смысл петлевой квантовой гравитации $L Q G$ и теории струн ST на базе теории взаимопрозрачных самосогласованных сетей в условиях гравитации в ${ }^{L} S T$ и антигравитации в ${ }^{D} S T$.

\begin{tabular}{|c|c|}
\hline$S T$ & ПІространство-в \\
\hline $\begin{array}{c}\text { Теория непрерывных ориентированных } \\
\text { сетей «эллиптического типа»с } \\
\text { линейным однородным расширением } \\
\text { в условиях гравитации }\end{array}$ & $\begin{array}{c}\text { Теория разрывных сетей } \\
\text { «гиперболического типа» с } \\
\text { анизотропным неоднородным сжатием } \\
\text { в условиях антигравитации }\end{array}$ \\
\hline $\begin{array}{l}\text { Как было указано ранее, гравитация в } \\
{ }^{L} S T \text { - является силой притяжения, } \\
\text { пытающаяся компенсировать } \\
\text { расширение тканей этого пространства } \\
\text { относительно неподвижных тканей }{ }^{A} S T \text {. } \\
\text { В начальный момент } B B \text { - она } \\
\text { минимальна, а своего максимума } \\
\text { достигает в момент } C r \text { (см. рис. } 1) . \\
\text { Моделирование пространства с } \\
\text { использованием сложных сетей - } \\
\text { является естественным процессом. } \\
\text { Попытки такого моделирования } \\
\text { строения геометрических ячеек тканей } \\
\text { пространства-времени }{ }^{L} S T \text { и их }\end{array}$ & $\begin{array}{l}\text { Как было указано ранее, } \\
\text { антигравитация в }{ }^{D} S T \text { - является силой } \\
\text { отталкивания, пытающаяся } \\
\text { компенсировать сжатие тканей этого } \\
\text { пространства относительно } \\
\text { неподвижных тканей }{ }^{A} S T . \\
\text { С точки зрения }{ }^{D} S T \text { (см. табл. } 3 \text { главы } \\
3.7) \text { в начальный момент } B B \text { - она } \\
\text { минимальна, а своего максимума } \\
\text { достигает в момент } C r \text { (см. рис. 1). Сила } \\
\text { антигравитации в начальный момент } \\
B B \text { разрывает саму ткань пространства- } \\
\text { времени, делая понятия: расстояния, } \\
\text { форму антиматерии и темного времени }\end{array}$ \\
\hline
\end{tabular}


взаимодействий (интерпретация автора) активно осуществляется в теории $L Q G[101,102]$, где Планковскую длину $l_{p}$ принимают за минимально возможное расстояние между узлами графов, которые образуют эластичную структуру сети тканей пространствавремени ${ }^{L} S T$, которая развивается во времени ${ }^{L} T$. Эта теория, которая должна быть согласованной с уравнениями ОТО - делает свои первые шаги. Но, как уже упоминалось в п. 6.4 настоящей Главы, для получения эффекта квантования и дальнейшего развития будущей теории гравитации необходимо учитывать, что каждый элемент этой сети фундаментально-сцеплен со своим партнером элементом сети в ${ }^{D} S T$, который имеет нетривиальную топологическую форму. Без учета фундаментальной-сцепленности тканей пространств-времен ${ }^{L} S T$ и ${ }^{D} S T$ развитие любой полноценной теории гравитации становится неэффективным.

\begin{abstract}
${ }^{D} T$ - предметом для дальнейших исследований. Необходимо также учитывать, что в условиях антигравитации расщепленные элементы тканей пространствавремени ${ }^{D} S T$ - должны: во-первых, постоянно находиться в движении, т.е. быть волнами, сохраняющими при этом свою топологическую целостность $(t-$ солитонами см. п. 6.8 и Главы 7 и 8), а во-вторых сохранять свойство фундаментальной сцепленности со своими партнерами в ${ }^{L} S T$. Оба этих фактора, а также факт того, что процесс сжатия ${ }^{D} S T$ в условиях антигравитации - является неоднородным и анизотропным, должны создавать топологический вращательный механизм, обеспечивающий стабилизацию и консенсус структурно-
\end{abstract} функциональных процессов в ${ }^{D} S T$ (далее, сокращенно: топологический вращательный механизм стабилизации).

String Theory, обладающая богатым и разнородным математическим аппаратом, с точки зрения автора, должна очень точно разделиться на две самостоятельные теории. Первую, так и назовем String Network Theory (SNT), которая начиная с работ $G$.Veneciano [103] и И. В. Волович [104], пытается интуитивно и на «ощупь» смоделировать строение антиматерии, сильного взаимодействия и тканей пространства-времени ${ }^{D} S T$, в условиях антигравитации. Целью использования в различных разновидностях этой теории многообразий Калаби-Яу, на взгляд автора, является не только компактизация скрытых измерений, но и попытка найти подобный эффективный топологический вращательный механизм стабилизации (или смысл этого многообразия), в условиях антигравитации. Косвенным подтверждением того, что многообразия Калаби-Яу относится к ${ }^{D} S T$ - является то, что при его выводе 


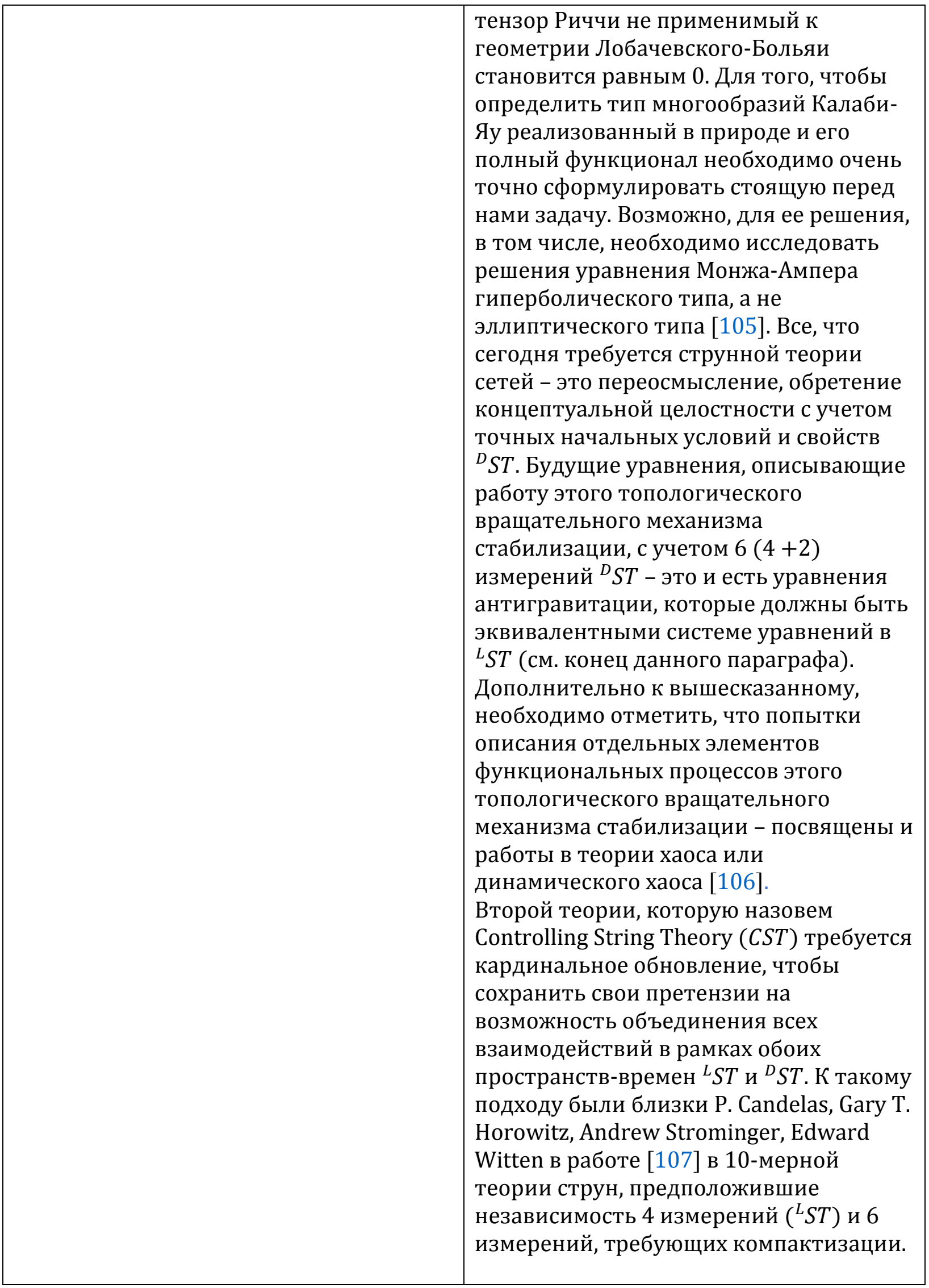

Описанные нами выше, алгоритмы расслоения ${ }^{A} S T$ предоставляет совершенно новые возможности для развития этих двух, взаимодополняющих друг друга, направлений на базе теории взаимопрозрачных самосогласованных сетей, имеющих 
антагонистические свойства. Во-первых, наличие первичного Абсолютного пространства-времени ${ }^{A} S T$, с геометрией Евклида $(\mathcal{E})$ с эталонной метрикой и неподвижной тканью - придает будущей теории фундаментальный характер. Вовторых, рождение в момент $B B$ и дальнейшее развитие двух самосогласованных пространств-времен ${ }^{L} S T$ и ${ }^{D} S T$ имеет точные зависимости. В-третьих, понятие фундаментальной сцепленности и эффекта спина (см. Главу 7) - накладывает ограничения на изменения и свойства пар «кривизна и расстояние», лежащих в основе строения тканей пространств-времен ${ }^{L} S T$ и ${ }^{D} S T$, придает будущей теории математическую строгость и ограждает исследователей от рассмотрения абстрактных, непродуктивных вариантов. Таким образом, осознанное и целенаправленное развитие теории взаимопрозрачных самосогласованных сетей, как физики строения и взаимодействий тканей пространств-времен ${ }^{L} S T$ и ${ }^{D} S T$ позволит в обозримом будущем, решить не только «проблему квантования гравитации», но и создать полноценную самосогласованную теорию гравитации и антигравитации. С учетом огромной сложности, стоящей перед нами задачи, ее можно разбить на три части:

1. Система самосогласованных уравнений в $T_{\text {synch }}$ : непрерывных ориентированных сетей «эллиптического типа» с линейным однородным расширением в ${ }^{L} S T$ и разрывных сетей «гиперболического типа» с неоднородным анизотропным сжатием в ${ }^{D} S T$ с использованием топологического вращательного механизма стабилизации.

2. Система самосогласованных уравнений в $T_{\text {synch }}$ : рождения масс ${ }^{L} m$ частиц материи из тканей сетей «эллиптического типа» в ${ }^{L} S T$ и масс ${ }^{D} m$ децентрализованных объектов антиматерии из тканей сетей «гиперболического типа» в ${ }^{D} S T$ с использованием топологических эффектов перепутывания тканей обоих пространств в ${ }^{A} S T$.

3. Система самосогласованных уравнений в $T_{\text {synch }}$, определяющих изменения параметров сети «эллиптического типа» в присутствии масс ${ }^{L} m$ материи в ${ }^{L} S T$ и определяющих изменения параметров сети «гиперболического типа» в присутствии масс ${ }^{D} m$ децентрализованных объектов антиматерии в ${ }^{D} S T$.

6.8 Форма материи в ${ }^{L} S T$ в условиях гравитации и форма антиматерии в ${ }^{D} S T$ в условиях антигравитации.

\begin{tabular}{|c|c|}
\hline Пространство-время ${ }^{L} S T$ & Пространство-время ${ }^{D} S T$ \\
\hline $\begin{array}{l}\text { Любая частица материи, рожденная в } \\
{ }^{L} S T \text { из тканей этого пространства, } \\
\text { испытывает действие гравитации или } \\
\text { взаимного притяжения со всеми } \\
\text { существующими объектами в }{ }^{L} S T . \\
\text { Собственно, именно эти } \\
\text { гравитационные взаимодействия и } \\
\text { делает ее форму округлой - превращая } \\
\text { в частицу. Процесс расширения тканей } \\
{ }^{L} S T \text { - является однородным на больших } \\
\text { масштабах, но их фундаментальная- }\end{array}$ & $\begin{array}{l}\text { Свойства антигравитации позволяют } \\
\text { выдвинуть гипотезу о форме } \\
\text { антиматерии в }{ }^{D} S T, \text { эквивалентной } \\
\text { частицам в }{ }^{L} S T . \\
\text { По мнению автора, такая форма в }{ }^{D} S T- \\
\text { является топологическим солитоном, } \\
\text { состоящим из пакета постоянно } \\
\text { распадающихся и двигающихся в } \\
\text { область своей локализации элементов } \\
\text { (см. главу 7). Для того чтобы отличать } \\
\text { топологические солитоны, как форму }\end{array}$ \\
\hline
\end{tabular}


сцепленность с тканями ${ }^{D} S T$ вынуждает находить различные формы для локального расширения. В связи с этим, любая частица - должна приобретать незначительную овальную форму (рано или поздно будут разработаны технологии, позволяющие очень точно исследовать формы частиц и это утверждение будет разрешено). В зависимости от стоящих перед нами задач, мы можем пренебрегать такой точностью формы у материи в микромире, например, считая элементарные частицы в ${ }^{L} S T$ точечными. антиматерии от вторичных солитонов в ${ }^{L} S T$, будем называть их сокращенно $\boldsymbol{t}$ солитонами, или солитонными облаками (s-облаками). Для данной гипотезы имеются следующие основания:

- $\quad{ }^{D} S T$ действует антигравитация;

- само ${ }^{D} S T$ неоднородно и анизотропно, что хорошо объясняет причину возникающих в нем нелинейных процессов;

- $\quad$ время ${ }^{D} T$ в ${ }^{D} S T$ также неоднородно и анизотропно (см. п. 5.1, 5.3 Главы 5), имеет волновую природу, что требует дальнейших исследований и идей для его описания;

- $\quad$ в ${ }^{D} S T$ реализуется геометрия $(\mathcal{L B})$;

- 5-й постулат утверждает, что в плоскости через точку, не лежащую на данной прямой, можно провести бесконечное количество линий, параллельных этой прямой;

- постоянно расщепляемые и двигающиеся в область своей локализации элементы децентрализованного объекта антиматерии ведут себя как единый, хорошо управляемый организм;

Таким образом, $\boldsymbol{t}$-солитоны в ${ }^{D} S T-$ являются формой антиматерии способной иметь и переносить элементарный топологический магнитный заряд, переносить энергию, импульс, момент импульса и т.д., сохраняя при этом свою целостность. Как и частицы в ${ }^{L} S T, \boldsymbol{t}$-солитоны в ${ }^{D} S T$ могут быть фермионами: заряженными или нейтральными, а также бозонами: нейтральными или заряженными.

С учетом вышеизложенного любая частица материи в ${ }^{L} S T$ будет фундаментальносцепленной с $t$-солитоном антиматерии в ${ }^{D} S T$. Полученный нами вывод о дуализме частиц и топологических солитонных волн с использованием двух самосогласованных пространств-времен ${ }^{L} S T$ и ${ }^{D} S T$ - является подтверждением основ квантовой механики (см. Главу 8). В частности, такой подход позволяет дать исчерпывающее объяснение всем разновидностям сил инерции, возникающим в ${ }^{L} S T$, убрав из обихода физики иррациональный термин «фиктивные силы». 


\section{Глава 7 Трех пространственно-временная природа спина ${ }_{t} S$}

\section{1 Вступление}

Прежде чем, предложить новую гипотезу о природе спина и дать ему определение, рассмотрим геометрические аспекты взаимодействий между пространствамивремени ${ }^{L} S T$ и ${ }^{D} S T$. На основе экспериментальных данных надежно установлено, что в любой точке (в $\left.{ }^{L} S T\right)$ векторы напряженности электрического $\vec{E}$ и магнитного полей $\vec{H}$ электромагнитной волны - взаимно перпендикулярны. Поскольку, в TSSCU носителями первичных элементарных топологических магнитных зарядов, порождающих первичное магнитное поле, являются позитрон и другие заряженные $t$-солитоны антиматерии, образующие позитронно-электрические волны в ${ }^{D} S T$, то мы можем предположить, что не только ЭМВ и ПЭВ, но и остальные фундаментальные взаимодействия между ${ }^{L} S T$ и ${ }^{D} S T$ в ${ }^{A} S T$ в $T_{\text {synch }}$ - происходят взаимоперпендикулярно. Косвенно, это же подтверждает и предложенная гипотеза о природе комплексных чисел (см. Главу 3) и использовании комплексной плоскости в математике. Таким-же образом, можно интерпретировать и нашу вторую гипотезу о темных комплексных числах в ${ }^{D} S T$ и темной комплексной плоскости (см. Главу 3 ).

Исходя из вышеизложенного, для выявления геометрической составляющей природы спина, воспользуемся известным фактом о том, что софокусные эллипсы и гиперболы в местах пересечения - взаимно перпендикулярны друг к другу (см рис. 9).

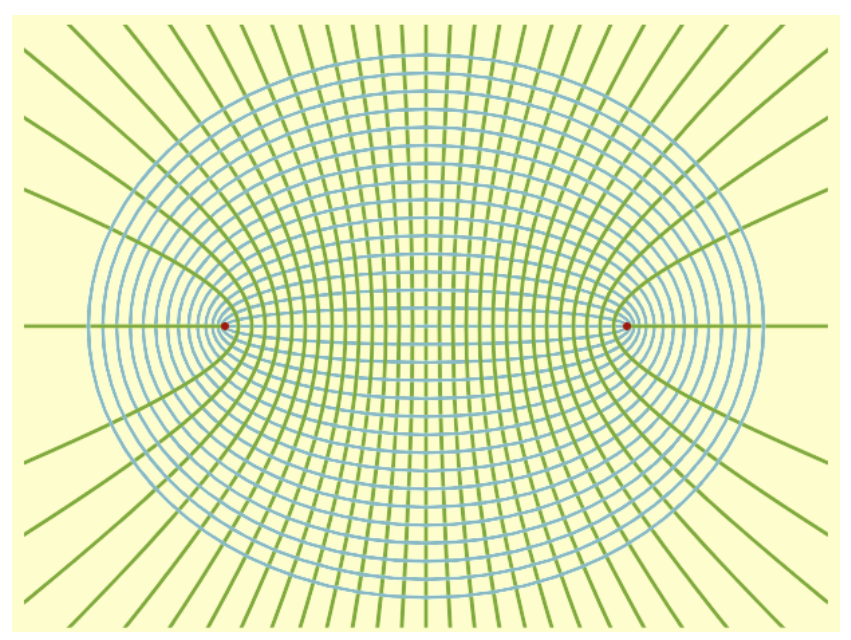

Рис. 9. Софокусные эллипсы и гиперболы. Хорошо известный математический факт о том, что пересечение софокусных эллипсов и гипербол всегда взаимоперпендикулярно, служит основой для определения геометрической составляющей понятия спина ${ }_{t} S$.

Забегая вперед, отметим что геометрическое расположение спина ${ }_{t} S$ в ${ }^{A} S T$ в момент $T_{\text {synch }}$ возможно, только в 4 (четырех) точках условного пересечения: траектории первичной (управляющей) частицы в ${ }^{A} S T$ - окружности, проекции траектории частицы ${ }^{L} M$ в ${ }^{L} S T$ на/в ${ }^{A} S T$ - эллипса, проекции софокусных с эллипсом - гипербол, 
как важнейших из всех возможных волновых траекторий (см. Главу 11) $t$-солитонов антиматерии ${ }^{D} M$ в ${ }^{D} S T$ на/в ${ }^{A} S T$ (см. рис.10). Обозначим верхнее левое пересечение буквой $A$, верхнее правое пересечение - буквой $B$, нижнее левое пересечение - буквой $C$ и нижнюю правое пересечение - буквой $D$.

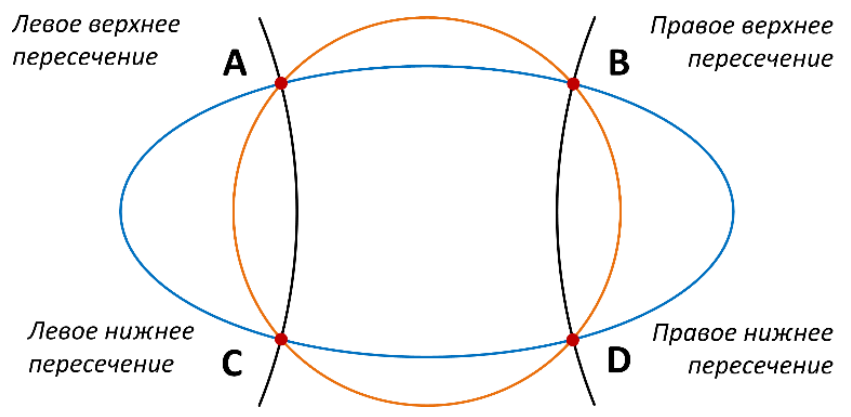

Рис. 10. Четыре возможных геометрических расположений спина ${ }_{t} S$, которые являются точками условных пересечений в трех пространствах-времени: окружности в ${ }^{A} S T$, эллипса в ${ }^{L} S T$ и проекций софокусных с эллипсом - гипербол в ${ }^{D} S T$ на/в ${ }^{A} S T$.

Эти 4 точки пересечения можно также называть точками максимального сближения проекций или точками локализации. Рассмотрим подробнее движение фундаментально-сцепленных электрона в ${ }^{L} S T$ и его партнера позитрона в ${ }^{D} S T$ в самосогласованном стационарном атоме.

\begin{tabular}{|l|l|}
\hline \multicolumn{1}{|c|}{ Пространство-время ${ }^{L} S T$} & \multicolumn{1}{|c|}{ Пространство-время ${ }^{D} S T$} \\
\hline $\begin{array}{l}\text { Пусть электрон (частица) в момент } \\
\text { времени }{ }^{L} T \text { находится в левой } \\
\text { верхней точке локализации }-A .\end{array}$ & $\begin{array}{l}\text { Пусть позитрон (частица) в момент } \\
\text { времени }{ }^{D} T \text { находится в левой } \\
\text { верхней точке локализации }-A .\end{array}$ \\
\hline
\end{tabular}

Согласно выдвигаемой нами гипотезе, точка локализации $A$ - является спином ${ }_{t} S_{A}$ или «окном прозрачности» в ${ }^{A} S T$ в $T_{\text {synch }}$, при котором между электроном (частицей) и позитроном (частицей) происходит «идеальное обменное взаимодействие», а времена ${ }^{L} T$ и ${ }^{D} T$ - синхронизируются. В TSSCU слово идеальное - добавляется, к известному в физике, обменному взаимодействию, которое, однако описано лишь частично [108, 109], без понимания его глубинного смысла. В момент спина ${ }_{t} S$ «идеальное обменное взаимодействие» происходит - без потерь (см. ниже определение спина).

- Далее, электрон (частица) движется, например, по часовой стрелке по траектории отличной от окружности и стремящейся к эллиптической, достигая точки локализации - D.
Далее, позитрон ( $t$-солитон) движется, против часовой стрелки, по гиперболическим $t$-солитонным траекториям, достигая точки локализации - D.

Точка локализации $D$ - является спином ${ }_{t} S_{D}$ или «окном прозрачности» в ${ }^{A} S T$ в $T_{\text {synch }}$, при котором между электроном (частицей) и позитроном (частицей) происходит «идеальное обменное взаимодействие», а времена ${ }^{L} T$ и ${ }^{D} T$ синхронизируются. 
- Продолжая движение по часовой стрелке по траектории отличной от окружности, но стремящейся к эллиптической, электрон (частица) снова достигает точки локализации - $A$.
- Продолжая движение против часовой стрелки по гиперболическим $t$-солитонным траекториям позитрон ( $t$-солитон) снова достигает точки локализации - $A$.

За один оборот по орбите отличной от окружности, но стремящейся к эллиптической в ${ }^{L} S T$ проекция электрона на/в ${ }^{A} S T$ дважды максимально сближается с проекцией позитрона в ${ }^{A} S T$. Позитрон, совершающий один оборот, по гиперболическим $t$-солитонным траекториям в ${ }^{D} S T$, также дважды максимально сближается до проекции электрона в ${ }^{A} S T$. Таким образом, спин электрона и позитрона, двигавшихся каждый в своем пространстве-времени от точки максимального сближения проекций $A$ к точке максимального сближения проекций $D$, и обратно, равен ${ }_{t} S_{(A \leftrightarrow D)}= \pm \frac{1}{2}$, как и полагается фермионам.

\section{2 Общее определение спина ${ }_{t} S$}

Спин ${ }_{t} S$ - это «окно прозрачности» возникающее в Абсолютном пространствевремени ${ }^{A} S T$ в $T_{\text {synch }}$ между частицей материи ${ }^{L} M$ фермионом (или частицей переносчиком взаимодействий бозоном) в пространстве-времени ${ }^{L} S T$ и ее партнером частицей антиматерии ${ }^{D} M$ фермионом (или частицей переносчиком взаимодействий темным бозоном) в пространстве-времени ${ }^{D} S T$ когда между фундаментальносцепленными частицей материи ${ }^{L} M$ и еe партнера частицей антиматерии ${ }^{D} M$ становится возможным:

- идеальное (без потерь) обменное взаимодействие;

- синхронизация времен ${ }^{L} T$ и ${ }^{D} T$;

Необходимо отметить, что идеальное обменное взаимодействие для фермионов и бозонов - имеет существенные отличия, о чем будет сказано ниже.

В TSSCU, для движения фундаментально-сцепленных безмассовых бозонов и свободных фермионов (вне атома или ядра), помимо стационарных замкнутых орбит, введенных еще И. Кеплером в 1609 году, будут рассмотрены также, осциллирующие незамкнутые орбиты (см. п. 7.3.3 и 7.4.2).

Таким образом, спин ${ }_{t} S$ - измеряется количеством моментов максимального сближения проекций, фундаментально-сцепленных частиц материи ${ }^{L} M \quad$ в пространстве-времени ${ }^{L} S T$ и их партнеров $t$-солитонов антиматерии ${ }^{D} M \quad$ в пространстве-времени ${ }^{D} S T$ на/в Абсолютное пространство-время ${ }^{A} S T$ в $T_{\text {synch }}$ за один

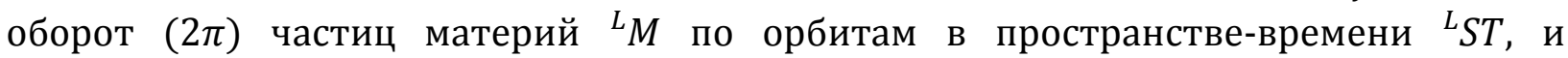
соответственно, за один оборот $(2 \pi) t$-солитонов антиматерии ${ }^{D} M$ по орбитам в пространстве-времени ${ }^{D} S T$.

$$
\text { Спин } t_{t} S=\frac{\text { один оборот частиц материй }{ }^{L} M \text { и } t-\text { солитонов }{ }^{D} M \text { по орбитам }}{\text { количество моментов максимального сближения проекций }}
$$


Необходимо отметить, что $t$-солитоны антиматерии ${ }^{D} M$ в ${ }^{D} S T$ в момент спина ${ }_{t} S$ всегда будет локализовываться в частицу, поскольку в ${ }^{A} S T$ реализовывается геометрия Евклида $(\mathcal{E})$ с постоянной гравитацией ${ }^{A} G_{r}=1$.

\section{3 Природа спина фермионов}

\subsection{1 Общие положения и определение}

Спин фермионов ${ }_{t} S= \pm \frac{1}{2}$, т.е. за один оборот $(2 \pi)$ по своим орбитам проекции: частиц материи ${ }^{L} M$ в пространстве-времени ${ }^{L} S T$, и соответственно, $t$-солитонов антиматерии ${ }^{D} M$ в пространстве-времени ${ }^{D} S T$ дважды максимально сближаются друг с другом:

$$
\text { Спин }{ }_{t} S=\frac{\text { один оборот фермионов материй }{ }^{L} M \text { и }{ }^{D} M \text { по орбитам }}{\text { количество моментов максимального сближения проекций }}= \pm \frac{1}{2}
$$

В современной физике спин ${ }_{t} S= \pm \frac{1}{2}$ измеряется в единицах $\hbar$, что является не совсем корректным. В действительности, с учетом формулы: ${ }^{L} E=\hbar \cdot \omega$ (где $\hbar=\frac{h}{2 \pi}$, а $\omega=f \cdot 2 \pi, h$ - постоянная Планка, $\hbar-$ постоянная Дирака, $f$ - частота, $\omega$ циклическая или угловая частота), это означает, что энергия фермионов в ${ }^{L} S T$ и ${ }^{D} S T$

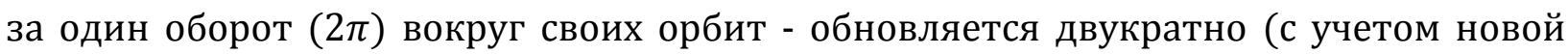
интерпретации квантовой механики (см. Главу 11), автор считает использование простой формулы ${ }^{L} E=\hbar \cdot \omega$ для фермионов вполне корректным).

Во время идеального обменного взаимодействия у фермионов происходят следующие пять операций:

1. Элементарный точечный электрический заряд электрона в ${ }^{L} S T$ преобразовывается в диполь в ${ }^{D} S T$ (т.е. у позитрона в ${ }^{D} S T$ есть собственный электрический момент);

2. Элементарный топологический магнитный заряд позитрона в ${ }^{D} S T$ преобразовывается в диполь в ${ }^{L} S T$ (т.е. у электрона в ${ }^{L} S T$ есть собственный магнитный момент);

3. Электрон и позитрон обмениваются своими импульсами ${ }^{L} P$ и ${ }^{D} P$;

4. Электрон и позитрон обмениваются своими информациями стохастической ${ }^{L} I$ и фрактальной ${ }^{D} I$, соответственно;

5. Происходит синхронизация времен ${ }^{L} T$ и ${ }^{D} T$.

Такое идеальное обменное взаимодействие «жизненно важными» параметрами, между фермионом в ${ }^{L} S T$ и его фундаментально-сцепленным партнером в ${ }^{D} S T$ не дает возможности двум и более фермионам в ${ }^{L} S T$ и ${ }^{D} S T$ находиться в одном и том же квантовом состоянии (принцип Паули), тем самым наделяя фермионы свойством образовывать структуру материи.

Как известно, существует $2 \cdot\left|{ }_{t} S\right|+1$ состояний спина. Следовательно, у фермионов возможны только 2 состояния: $+\frac{1}{2}$ и $-\frac{1}{2}$.

Деление значения спина по положительному и отрицательному знаку ${ }_{t} S= \pm \frac{1}{2}$, понятие спиральности (требующее точного определения) и киральности не является предметом изложения данной статьи и могут быть рассмотрены в одной из 
последующих публикаций. Во всех случаях необходимы формулы, описывающие движение $t$-солитонов в ${ }^{D} S T$, а также формулы, описывающие согласованное движение обоих фундаментально-сцепленных фермионов (см. Главу 11).

Напомним, что фундаментально-сцепленные фермионы, входящие в состав самосогласованного атома, двигаются по стационарным замкнутым эллиптическим и гиперболическим $t$-солитонным орбитам в противоположных радиальных направлениях (см. рис. 11-14). А свободные фундаментально-сцепленные фермионы (вне атома или ядра) двигаются по осциллирующим незамкнутым эллиптическим и гиперболическим $t$-солитонным орбитам (см. рис. 15). Рассмотрим ниже, все эти возможные комбинации.

\subsection{2 Геометрическое расположение спина фундаментально-сцепленных фермионов в самосогласованном атоме}

Рассмотрим случай, когда для наблюдателя N1, электрон двигается по часовой стрелке по эллиптической орбите. В этом случае позитрон ( $t$-солитон) будет двигаться против часовой стрелки (например, в атоме водорода, по $s$-орбитали) по гиперболическим $t$-солитонным траекториям (см. рис. 11 и рис. 12). В этом случае, единственной возможной геометрической комбинацией, при которой возможен эффект спина будет: левая верхняя точка локализации - $A$ и правая нижняя точка локализации $-D$, что можно записать, как ${ }_{t} S_{(A \leftrightarrow D)}= \pm \frac{1}{2}$.
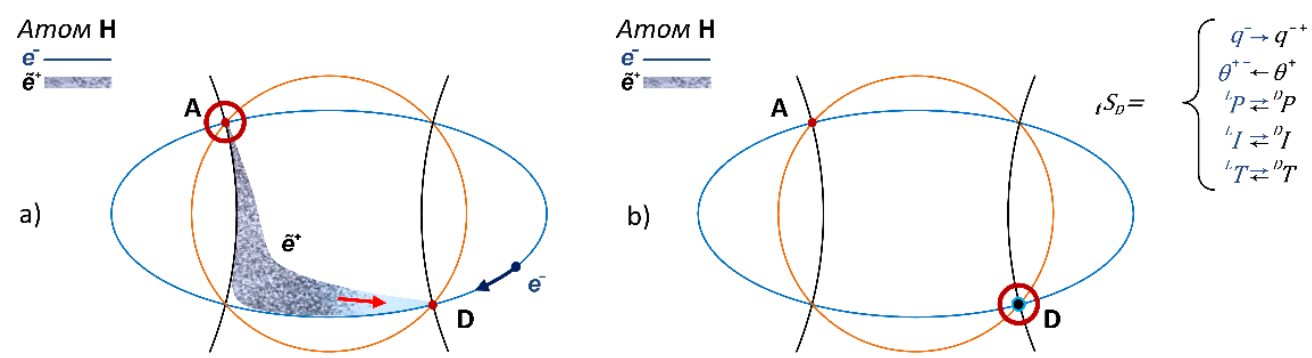

Рис. 11 (a и b). Концепция движения фундаментально-сцепленных фермионов в самосогласованном частице-солитонном атоме водорода протии. На рисунке 11a слева изображены: как электрон (частица) двигающийся, по часовой стрелке, по траектории отличной от окружности и стремящейся к эллиптической в ${ }^{L} S T$ и позитрон ( $t$-солитон) двигающийся, против часовой стрелки (по $s$-орбитали), по гиперболическим $t$-солитонным траекториям в ${ }^{D} S T$ - приближаются к точке локализации - D $\left({ }_{t} S_{D}= \pm \frac{1}{2}\right)$ в ${ }^{A} S T$. На рисунке $11 \mathrm{~b}$ справа показан момент максимального сближения проекций электрона (частицы) и позитрона (частицы) в правой нижней точке локализации - $D\left({ }_{t} S_{D}= \pm \frac{1}{2}\right)$ в ${ }^{A} S T$, когда между электроном частицей и позитроном частицей - становится возможным идеальное обменное взаимодействие. 

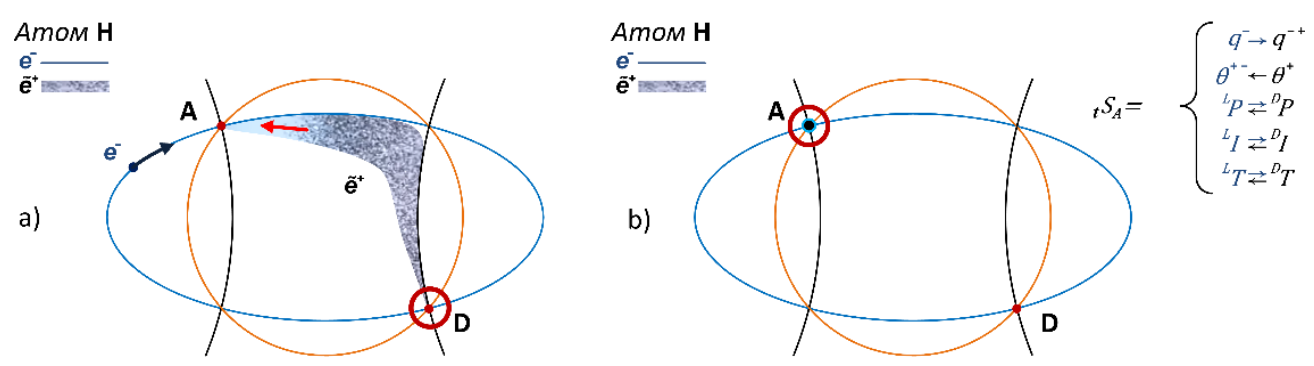

Рис. 12 (a и b). Концепция движения фундаментально-сцепленных фермионов в самосогласованном частице-солитонном атоме водорода протии. На рисунке $12 \mathrm{a}$ слева изображены: как электрон (частица) двигающийся, по часовой стрелке, по траектории отличной от окружности и стремящейся к эллиптической в ${ }^{L} S T$ и позитрон ( $t$-солитон) двигающийся, против часовой стрелки (по $s$-орбитали), по гиперболическим $t$-солитонным траекториям в ${ }^{D} S T$ - приближаются к точке локализации - $A\left({ }_{t} S_{A}= \pm \frac{1}{2}\right)$ в ${ }^{A} S T$. На рисунке $12 \mathrm{~b}$ справа показан момент максимального сближения проекций электрона (частицы) и позитрона (частицы) в левой верхней точке локализации - $A\left({ }_{t} S_{A}= \pm \frac{1}{2}\right)$ в ${ }^{A} S T$, когда между электроном частицей и позитроном частицей - становится возможным идеальное обменное взаимодействие.

Для наблюдателя N2, находящегося напротив наблюдателя N1, электрон двигается против часовой стрелки по эллиптической орбите, а позитрон ( $t$-солитон) двигается по часовой стрелке (по $s$-орбитали), по гиперболическим $t$-солитонным траекториям (см. рис. 13 и рис. 14). В этом случае геометрическое расположение спина будет: в правой верхней точке локализации $-B$ и левой нижней точкой локализации - $C$, что можно записать, как ${ }_{t} S_{(B \leftrightarrow C)}= \pm \frac{1}{2}$.
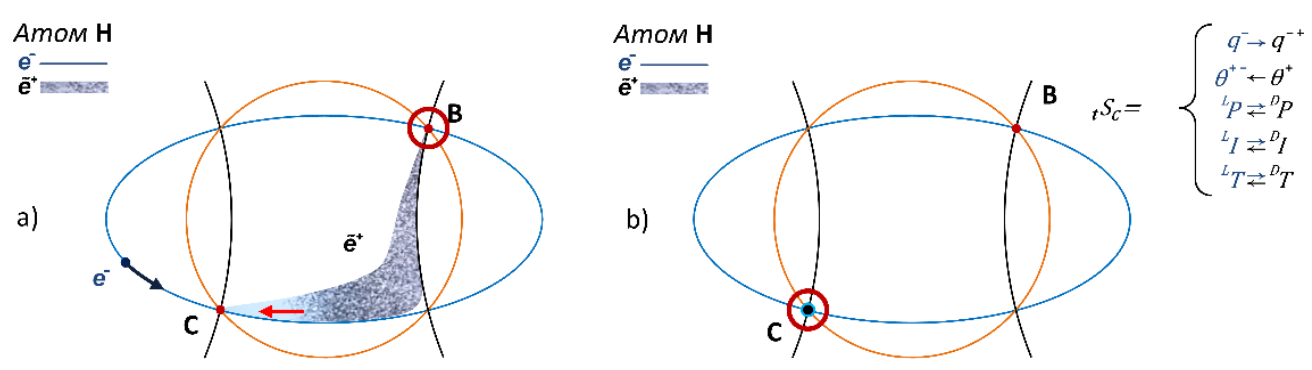

Рис. 13 (a и b). Концепция движения фундаментально-сцепленных фермионов в самосогласованном частице-солитонном атоме водорода протии. На рисунке 13a слева изображены: как электрон (частица) двигающийся, против часовой стрелки, по траектории отличной от окружности и стремящейся к эллиптической в ${ }^{L} S T$ и позитрон ( $t$-солитон) двигающийся, по часовой стрелке (по $s$-орбитали), по возможным гиперболическим $t$-солитонным траекториям в ${ }^{D} S T$ - приближаются к точке локализации - $C\left({ }_{t} S_{C}= \pm \frac{1}{2}\right)$ в ${ }^{A} S T$. На рисунке $13 \mathrm{~b}$ справа показан момент максимального сближения проекций электрона (частицы) и позитрона (частицы) в нижней левой точке локализации - $C\left({ }_{t} S_{C}= \pm \frac{1}{2}\right)$ в ${ }^{A} S T$, когда между электроном частицей и позитроном частицей - становится возможным идеальное обменное взаимодействие. 

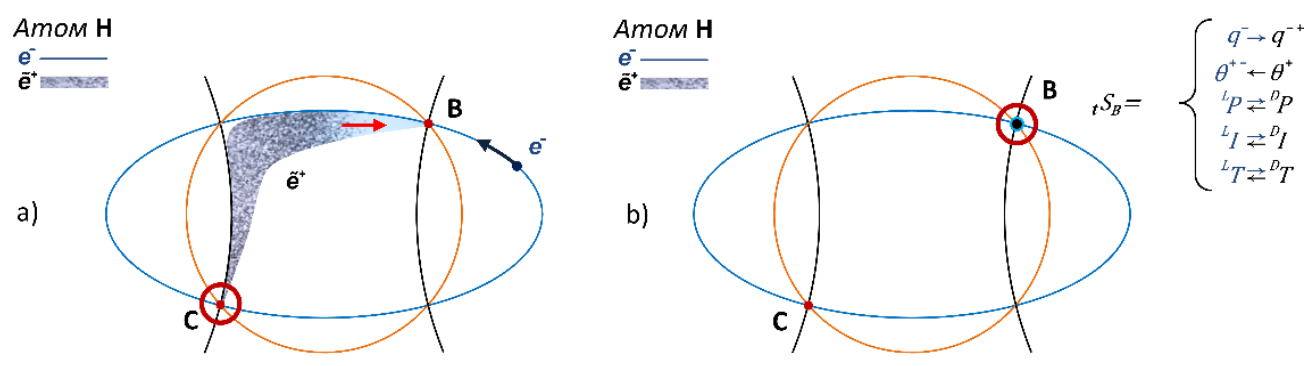

Рис. 14 (a и b). Концепция движения фундаментально-сцепленных фермионов в самосогласованном частице-солитонном атоме водорода протии. На рисунке 14a слева изображены: как электрон (частица) двигающийся, против часовой стрелки, по траектории отличной от окружности и стремящейся к эллиптической в ${ }^{L} S T$ и позитрон ( $t$-солитон) двигающийся, по часовой стрелке (по $s$-орбитали), по возможным гиперболическим $t$-солитонным траекториям в ${ }^{D} S T$ - приближаются к точке локализации - $B\left({ }_{t} S_{B}= \pm \frac{1}{2}\right)$ в ${ }^{A} S T$. На рисунке $14 \mathrm{~b}$ справа показан момент максимального сближения проекций электрона (частицы) и позитрона (частицы) в правой верхней точке локализации - $B\left({ }_{t} S_{B}= \pm \frac{1}{2}\right)$ в ${ }^{A} S T$, когда между электроном частицей и позитроном частицей - становится возможным идеальное обменное взаимодействие.

Таким образом, приведенные на рисунках 11-12 и 13-14 примеры движения фундаментально-сцепленных электрона и позитрона (по s-орбитали) в самосогласованном частице-солитонном атоме - являются лево-право симметричными.

\subsection{3 Геометрическое расположение спина при движении свободных (вне атома или ядра) фундаментально-сцепленных фермионов по осциллирующим незамкнутым орбитам}

Свободные фундаментально-сцепленные фермионы вне атома или ядра двигаются согласованно, каждый в своем пространстве-времени по осциллирующим незамкнутым орбитам. В простейшем одномерном случае такие осциллирующие незамкнутые эллиптические орбиты в ${ }^{L} S T$ можно определить, как траектории, состоящие из кривых, отображение которых с использованием зеркальной симметрии необходимое количество раз - позволяет нам замкнуть орбиту на самою себя (см. рис. 15). Для определения сложных осциллирующих незамкнутых гиперболических $t$-солитонных орбит в ${ }^{D} S T$ требуются дальнейшие исследования и точные формулы. Но главное, что для реализации эффекта спина $t$-солитоны также всегда двигаются из одной точки локализации к другой. При этом пара точек локализации осциллирующих фундаментально-сцепленных фермионов ${ }_{t} S_{A \leftrightarrow C}= \pm \frac{1}{2}$ или ${ }_{t} S_{B \leftrightarrow D}= \pm \frac{1}{2}$ - будут каждый раз находиться в новых областях пространстввремен ${ }^{A} S T,{ }^{L} S T$ и ${ }^{D} S T$.

Необходимо сразу оговориться, что на рис. 15 ( $\mathrm{a}$ и b) представленным далее, показана лишь концепция двух возможных гиперболических $t$-солитонных орбит для позитрона с использованием одной ветви гиперболы, имеющих разную спиральность. 


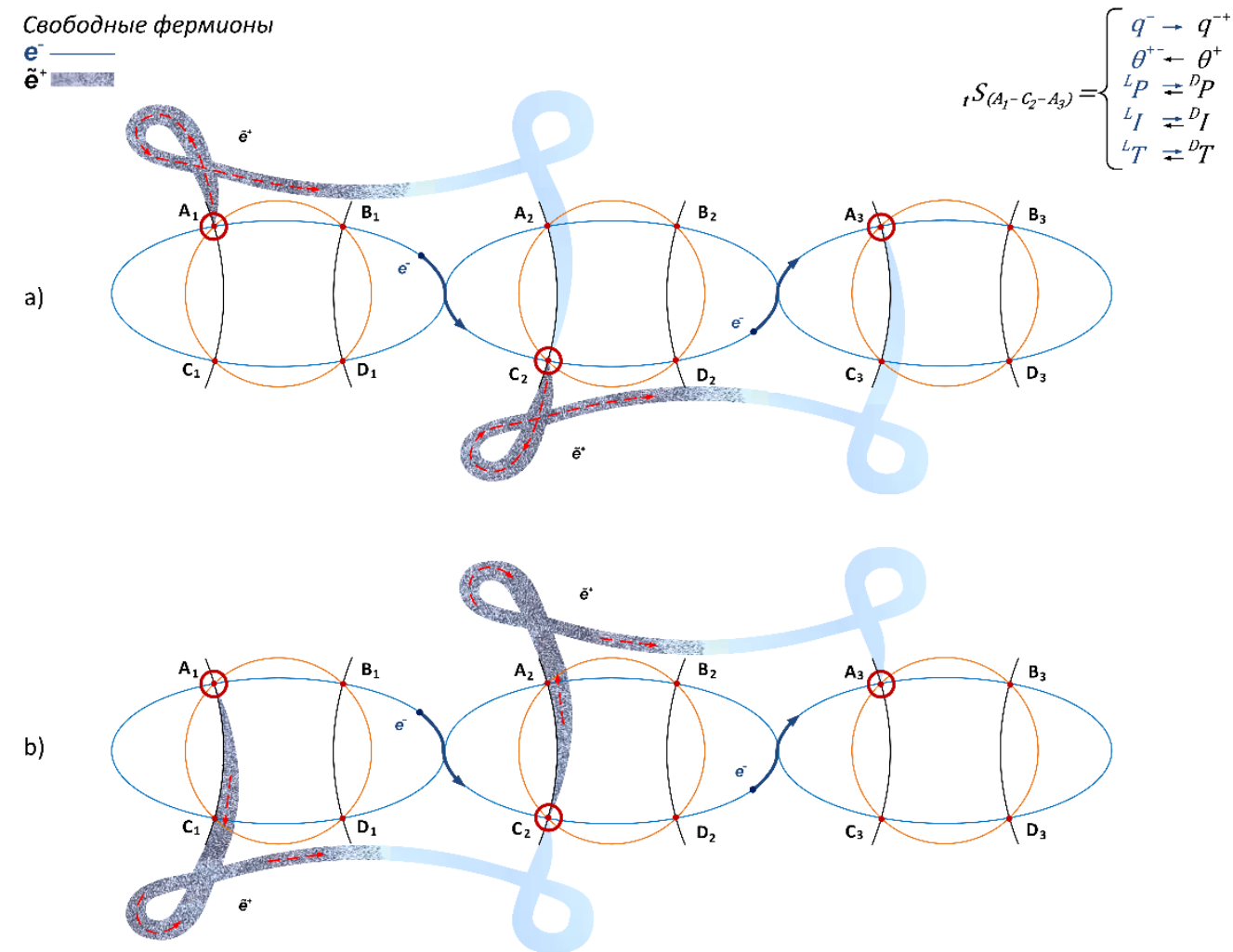

Рис. 15 (a и b). Концепция движения свободных (вне атома или ядра) фундаментально-сцепленных фермионов: электрона (частицы) по осциллирующим незамкнутым эллиптическим орбитам в ${ }^{L} S T$ и позитрона ( $t$-солитона) по двум возможным осциллирующим незамкнутым гиперболическим $t$-солитонным орбитам в ${ }^{D} \boldsymbol{S T}$ с использованием одной ветви гиперболы. Каждая из точек локализации $A_{1}, C_{2}$ и $A_{3}$ - является окном прозрачности или спином ${ }_{t} S$, когда становится возможным идеальное обменное взаимодействие между электроном частицей и позитроном частицей. На обоих частях рис. 15 (a и b) траектории электрона в ${ }^{L} S T$ - совпадают. На рис. 15a изображено движение позитрона ( $t$-солитона) в ${ }^{D} S T$ по осциллирующей незамкнутой возможной гиперболической $t$-солитонной орбите из точки локализации $A_{1}$ в точку локализации $C_{2}$ и далее в точку локализации $A_{3}$ (с 2 петлями за пол оборота), при которой «спиральность» можно интерпретировать как положительную. На рис. $15 \mathrm{~b}$ изображено движение позитрона ( $t$-солитона) в ${ }^{D} S T$ по осциллирующей незамкнутой возможной гиперболической $t$-солитонной орбите из точки локализации $A_{1}$ в точку локализации $C_{2}$ и далее в точку локализации $A_{3}$ (с 2 петлями за пол оборота), при которой «спиральность» можно интерпретировать как отрицательную.

Необходимо отметить, что траектории свободных фундаментально-сцепленных фермионов: электрона и позитрона - не являются лево-право симметричными.

\section{4 Природа спина бозонов}

\subsection{1 Общие положения и определение}

Бозоны подразделяются на два класса: безмассовые бозоны (фотоны $\gamma$ в ${ }^{L} S T$ и темные фотоны $\tilde{\gamma}$ в $\left.{ }^{D} S T\right)$ не имеющие зарядов, и массивные векторные бозоны $\left({ }^{+} W,{ }^{-} W,{ }^{0} Z\right)$ среди которых ${ }^{ \pm} W$ - являются заряженными. Спин всех бозонов ${ }_{t} S= \pm 1$, но идеальное обменное взаимодействие у безмассовых нейтральных и у массивных, 
имеющих заряды бозонов - существенно различаются. Остановимся на безмассовых нейтральных бозонах, поскольку ${ }^{ \pm} W$, возникающие при слабом взаимодействии являются исключением из правил и будут рассмотрены в конце этого раздела.

Итак, спин бозонов ${ }_{t} S= \pm 1$, т.е. за один оборот $(2 \pi)$ по своим осциллирующим незамкнутым орбитам проекции фундаментально-сцепленных частиц материи ${ }^{L} M$ в пространстве-времени ${ }^{L} S T$, и соответственно, $t$-солитонов антиматерии ${ }^{D} M$ в пространстве-времени ${ }^{D} S T$ один раз максимально сближаются друг с другом:

$$
\text { Спин }{ }_{t} S=\frac{\text { один оборот бозонов материй }{ }^{L} M \text { и темных бозонов }{ }^{D} M \text { по орбитам }}{\text { количество моментов максимального сближения проекций }}= \pm 1
$$

В современной физике спин ${ }_{t} S= \pm 1$ измеряется в единицах $\hbar$, что является не совсем корректным. В действительности, с учетом формулы: ${ }^{L} E=\hbar \cdot \omega$ (где $\hbar=\frac{h}{2 \pi}$, а $\omega=f \cdot 2 \pi, h$ - постоянная Планка, $\hbar$ - постоянная Дирака, $f$ - частота, $\omega$ - циклическая или угловая частота), это означает, что энергия безмассовых нейтральных бозонов за один оборот $(2 \pi)$ вокруг своих орбит - не меняется.

Во время идеального обменного взаимодействия у фотона $\gamma$ и фундаментальносцепленного с ним темного фотона $\tilde{\gamma}$ происходят следующие две операции:

1. Бозоны обмениваются своими информациями стохастической ${ }^{L} I$ и фрактальной ${ }^{D} I$, соответственно;

2. Происходит синхронизация времен ${ }^{L} T$ и ${ }^{D} T$.

Безмассовые бозоны, в отличии от заряженных фермионов, не обладают зарядами и не обмениваются своими импульсами ${ }^{L} P$ и ${ }^{D} P$. Эти отличия и спин ${ }_{t} S= \pm 1$, позволяют множеству безмассовых нейтральных бозонов в ${ }^{L} S T$ находиться в одном и том же квантовом состоянии, тем самым, наделяя их свойством - являться переносчиками взаимодействий.

В TSSCU фотоны $\gamma$ и темные фотоны $\tilde{\gamma}$ - являются фундаментально-сцепленными.

Исходя из предлагаемой гипотезе о природе спина, пп. 5.10, 5.11 Главы 5 и рассмотренных выше заключений можно уверенно сделать предсказания:

- частиц, которые являются античастицами сами для себя - не существует;

- безмассовый нейтральный бозон фотон - $\gamma$ в пространстве-времени ${ }^{L} S T$, имеющий спин ${ }_{t} S= \pm 1$ - обязан иметь фундаментально-сцепленного партнера: безмассового нейтрального темного фотона - $\tilde{\gamma}$ в пространстве-времени ${ }^{D} S T$, также со спином ${ }_{t} S= \pm 1$.

При этом фотоны $\gamma$ являются переносчиками квантов ${ }^{L} \hbar$ электромагнитного взаимодействия, а темные фотоны $\tilde{\gamma}$ - являются переносчиками темных квантов $D_{\hbar_{1-5}}$ позитронноэлектрического (сильного) взаимодействия. Гипотеза (возможность) о существовании у фотона волновой функции была выдвинута (рассмотрена) еще в 1930 году $[110,111]$, но столкнулась с серьезными теоретическими трудностями. Открытие оптических солитонов [112] позволяет надеяться на решение проблем вычисления самосогласованного движения фотонов и темных фотонов - в ближайшее время. 
Как известно, существует $2 \cdot\left|{ }_{t} S\right|+1$ состояний спина. Следовательно, у бозонов возможны - 3 состояния: $+1,-1$ и, так называемое, 0 . Деление значения спина по положительному и отрицательному знаку ${ }_{t} S= \pm 1$, а также точное определение понятия спиральности - станет окончательно ясным только после получения формул, описывающих согласованное движение фундаментально-сцепленных бозонов. Рассмотрим примеры их возможного согласованного движения.

\subsection{2 Геометрическое расположение спина при движении фундаментально- сцепленных безмассовых бозонов по осциллирующим незамкнутым орбитам}

При движении фундаментально-сцепленной частицы фотона в ${ }^{L} S T$ из 4 возможных геометрических точек расположения спина (см. рис. 10), в одном направление он проходит большую часть эллиптической траектории (по данному эллипсу), а в другом направлении меньшую часть эллиптической траектории (по данному эллипсу), см. рис. 16 (а и b). С учетом спина ${ }_{t} S= \pm 1$ фотон $\gamma$ в ${ }^{L} S T$ проходит один оборот по осциллирующей незамкнутой эллиптической траектории до следующей точки локализации и идеального обменного взаимодействия.

Необходимо сразу оговориться, что на рис. 16 (a и b) представленным далее, показана лишь концепция двух возможных гиперболических $t$-солитонных орбит для темного фотона $\tilde{\gamma}$, с использованием ветвей обеих гипербол, имеющих разную спиральность. 


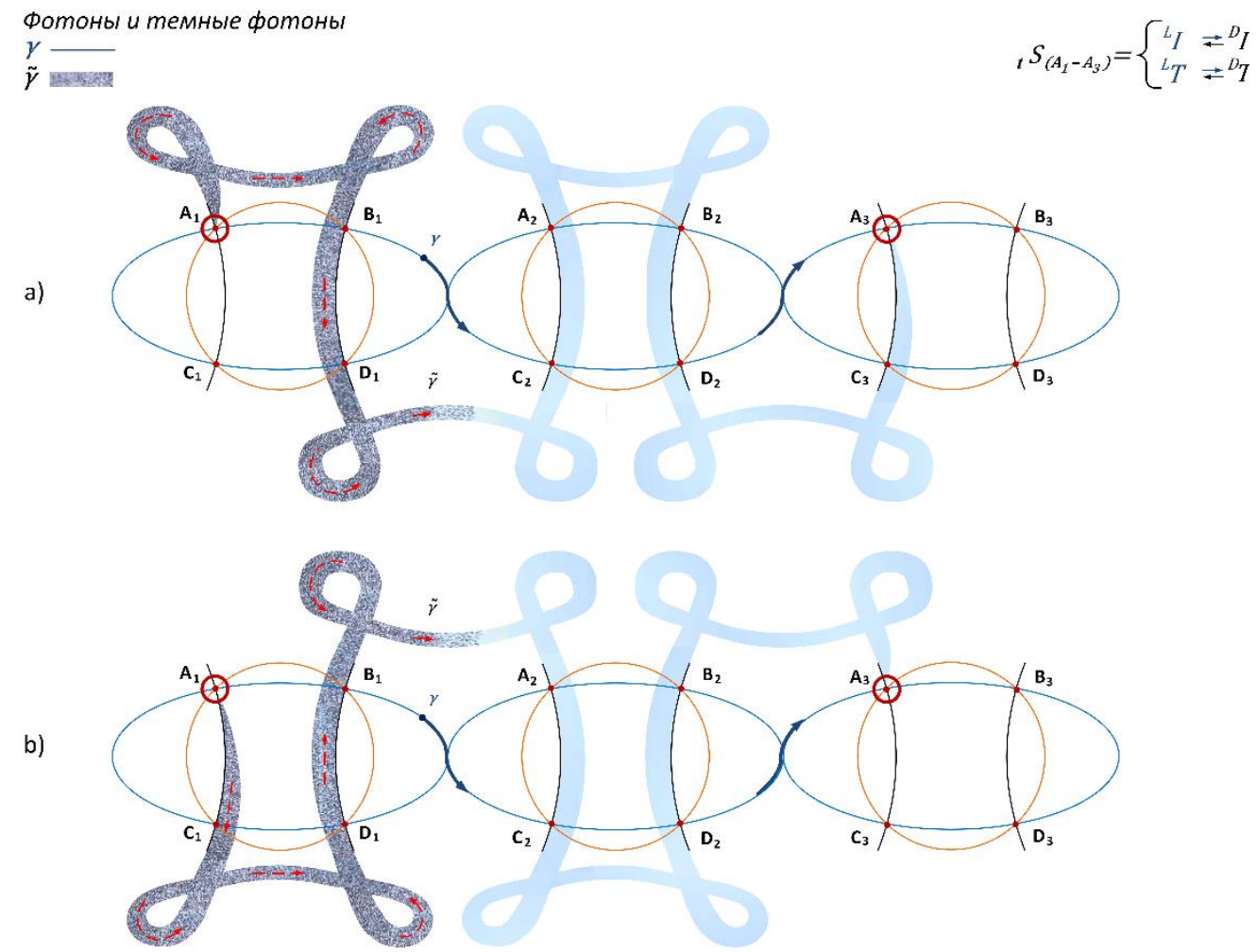

Рис. 16 (a и b). Концепция движения фундаментально-сцепленных бозонов: фотона (частицы) по осциллирующим незамкнутым эллиптическим орбитам в ${ }^{L} S T$ и темного фотона ( $t$-солитона) по двум возможным осциллирующим незамкнутым гиперболическим $t$-солитонным орбитам в ${ }^{D} S T$ с использованием ветвей обеих гипербол. Точки локализации $A_{1}$ и $A_{3}$ - являются окнами прозрачности или спином ${ }_{t} S$, когда становится возможным идеальное обменное взаимодействие между фотоном частицей и темным фотоном частицей. На обоих частях рис. 16 (a и b) траектории фотона - совпадают. На рис. 16 (a и b) изображены движения темного фотона ( $t$-солитона) в ${ }^{D} S T$ по двум различным осциллирующим незамкнутым возможным гиперболическим $t$-солитонным орбитам из точки локализации $A_{1}$ в точку локализации $A_{3}$, имеющим разную спиральность.

Необходимо отметить, что движения фундаментально-сцепленных бозонов: фотона и темного фотона - не являются лево-право симметричными.

Третье состояние спина ${ }_{t} S$ бозонов, так называемое 0 - запрещено для фотонов и темных фотонов. Причина этого запрета будет рассмотрена в следующей статье «Формализм TSSCU».

\subsection{3 Особенности спина при движении фундаментально-сцепленных массивных векторных ${ }^{ \pm} W$ бозонов}

Для заряженных ${ }^{ \pm} W$ массивных векторных бозонов идеальное обменное взаимодействие будет происходить аналогично тому, как оно проходит у фермионов, с той лишь существенной разницей, что оно у ${ }^{ \pm} W$ бозонов происходит один раз за оборот $(2 \pi)$ вокруг своих орбит. Эта разница делает заряженные массивные векторные ${ }^{ \pm} W$ бозоны - нестабильными и короткоживущими, что позволяет им играть свою надлежащую роль переносчиков слабого взаимодействия. 


\subsection{4 Особенности спина массивного векторного ${ }^{0} Z$ бозона. Предсказание.}

Нам остается рассмотреть нейтральный массивный векторный ${ }^{0} Z$ бозон, у которого также должно происходить идеальное обменное взаимодействие со своим фундаментально-сцепленным партнером из антиматерии. Но оказалось, что его партнер темный бозон ${ }^{0} \tilde{Z}$ из антиматерии - до сих пор не найден. Хотелось бы однозначно заявить, что приведенная гипотеза о природе спина - исключает такую возможность. Исходя из этого, можно уверенно сделать следующие предсказания:

- частиц, которые являются античастицами сами для себя - не существует;

- массивный бозон ${ }^{0} Z$ в пространстве-времени ${ }^{L} S T$, имеющий спин ${ }_{t} S= \pm 1$ - обязан иметь фундаментально-сцепленного партнера нейтрального массивного бозона из антиматерии - ${ }^{0} \tilde{Z}$ в пространстве-времени ${ }^{D} S T$, также со спином ${ }_{t} S= \pm 1$.

\subsection{5 Спин бозона Хиггса $H$}

Особенности бозона Хиггса $H$, имеющего нулевой спин $\left({ }_{i} S=0\right)$ и скалярного поля Браута-Энглера-Хиггса $(B E H)$ будут рассмотрены в следующей статье «Формализм TSSCU».

\section{5 Заключение}

Спин ${ }_{t} S$ - является фундаментальным механизмом или эффектом, обеспечивающим идеальное обменное взаимодействие и синхронизацию времен ${ }^{L} T$ и ${ }^{D} T$ между фундаментально-сцепленными частицами в ${ }^{L} S T$ и их партнерами $t$-солитонами в ${ }^{D} S T$. 


\section{Глава 8 Самосогласованная частице-солитонная модель атома водорода (протия) в трех пространствах-времени ${ }^{A} S T,{ }^{L} S T,{ }^{D} S T$}

Рассмотрим самосогласованную частице-солитонную модель простейшего атома водорода - протия. Напомним, что протий не содержит нейтральных частиц и состоит из ядра и элементарной частицы, двигающейся вокруг него.

Для каждого из трех пространств-времен частная модель, описывающая протий, будет иметь свои существенные отличия, обусловленные разностью свойств этих пространств-времен, а также фундаментальным механизмом спина.

В Абсолютном пространстве-времени ${ }^{A} S T$ атом первичного (или управляющего) протия представляет собой первичный электрон, который движется по идеальной окружности вокруг первичного протона, состоящего из трех первичных кварков, поскольку в ${ }^{A} S T$ действуют законы геометрии Эвклида и постоянная гравитация.

\begin{tabular}{|c|c|}
\hline Пространство-время ${ }^{L} S T$ & Пространство-время ${ }^{D} S T$ \\
\hline $\begin{array}{l}\text { - Атом и ядро протия включает в себя } \\
\text { протон - } p \text {, состоящий из трех } \\
\text { кварков: }(u, u, d) \text { и электрона - } e, \\
\text { который движется по орбите } \\
\text { отличной от окружности и } \\
\text { стремящейся к эллиптической } \\
\text { вокруг протона - } p \text {, поскольку в }{ }^{L} S T \\
\text { реализуется геометрия Римана и } \\
\text { положительная гравитация. }\end{array}$ & $\begin{array}{l}\text { - Атом и ядро антипротия включает в } \\
\text { себя антипротон - } \tilde{p}, \text { состоящий из } \\
\text { трех антикварков: }(\tilde{u}, \tilde{u} \tilde{d}) \text { и } \\
\text { позитрон - } \tilde{e}, \text { который движется } \\
\text { вокруг антипротона - } \tilde{p} \text { по } \\
\text { гиперболическим } t \text {-солитонным } \\
\text { траекториям, поскольку в }{ }^{D} S T \\
\text { реализуется геометрия } \\
\text { Лобачевского-Больяи и } \\
\text { отрицательная гравитация. }\end{array}$ \\
\hline $\begin{array}{l}\text { - } \text { Рассмотрим движение электрона } e^{-} \\
\text {(частицы) вокруг протона } p^{+} \\
\text {(частицы), подробнее. Как известно } \\
\text { электрон } e^{-} \text {обладает элементарным } \\
\text { точечным электрическим } \\
\text { отрицательным зарядом } q^{-} \text {равным } \\
\text { по модулю положительному } \\
\text { точечному заряду } q^{+} \text {протона } p^{+} ;\end{array}$ & $\begin{array}{l}\text { • } \\
\text { Рассмотрим движение позитрона } \tilde{e}^{+} \\
\left(t \text {-солитона) вокруг антипротона } \tilde{p}^{-}\right. \\
(t \text {-солитона) подробнее. Мы } \\
\text { предполагаем, что позитрон } \\
\text { обладает элементарным } \\
\text { топологическим магнитным } \\
\text { положительным зарядом - } \tilde{\theta}^{+} \\
\text {равным по модулю, отрицательному } \\
\text { элементарному топологическому } \\
\text { магнитному заряду } \tilde{\theta}^{-} \text {антипротона } \\
\tilde{p}^{-} ; \\
\text {В }{ }^{D} S T t \text {-солитоны антиматерии, } \\
\text { имеющие разноименные } \\
\text { топологические магнитные заряды - } \\
\text { отталкиваются. }\end{array}$ \\
\hline
\end{tabular}

Таким образом, в любой момент $T_{\text {synch }}$ в ${ }^{A} S T$ (см. рис. 17) сила притяжения ${ }^{L} F$ между отрицательно электрически заряженным электроном $e^{-}$и положительно электрически заряженным протоном $p^{+}{ }_{\text {в }}{ }^{L} S T$ компенсируется совокупными силами 
отталкивания ${ }^{D} F_{i j}$ между положительно магнито заряженным позитроном $\tilde{e}^{+}$и отрицательно магнито заряженным антипротоном $\tilde{p}^{-}$в ${ }^{D} S T$. В результате, в ${ }^{A} S T$ между фундаментально-сцепленными партнерами электроном $e^{-}$и позитроном $\tilde{e}^{+}$и между фундаментально-сцепленными партнерами протоном $p^{+}$и антипротоном $\tilde{p}^{-}-$ возникает динамическое равновесие, которое не позволяет электрону $e^{-}$упасть на протон $p^{+}{ }_{\text {в }}^{L} S T$, а позитрону $\tilde{e}^{+}$оторваться от антипротона $\tilde{p}^{-}{ }_{\text {в }}{ }^{D} S T$.

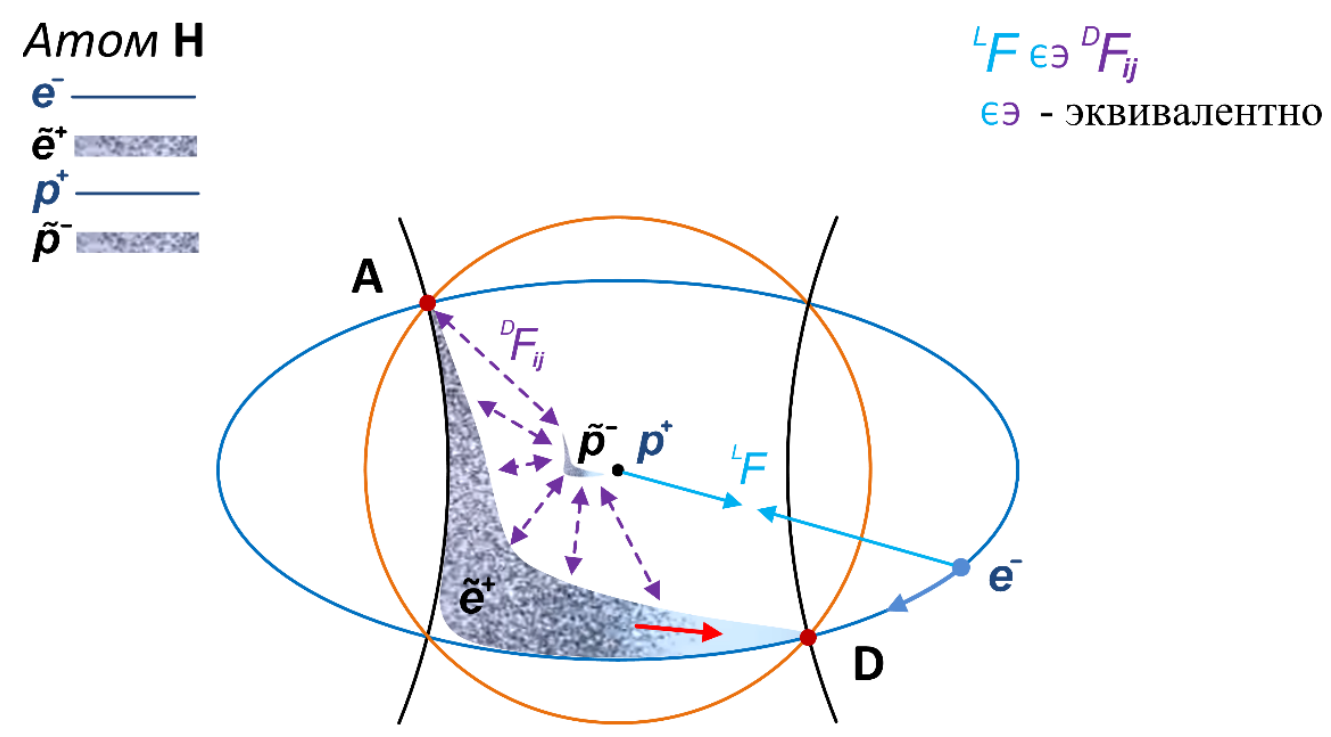

Рис. 17. Самосогласованная частице-солитонная модель атома водорода (протия) в трех пространствах-времени. В любой момент $T_{\text {synch }}$ в ${ }^{A} S T$ электрическая сила притяжения ${ }^{L} F\left({ }^{L} t\right)$, действующая между электроном (частицей) и протоном (частицей) в ${ }^{L} S T$ - эквивалентна совокупным магнитным силам отталкивания ${ }^{D} F_{i j}\left({ }^{D} t\right)$, действующим между позитроном ( $t$-солитоном) и антипротоном $\left(t\right.$-солитоном) в ${ }^{D} S T$. В общем случае ядра (протон и антипротон) не обязаны находиться в центре и размещены там - для наглядности. 
Глава 9 Фундаментальные взаимодействия в пространствахвремени ${ }^{L} S T$ и ${ }^{D} S T$

\begin{tabular}{|c|c|}
\hline Пространство-время ${ }^{L} S T$ & Пространство-время ${ }^{D} S T$ \\
\hline $\begin{array}{l}\text { • } \\
\text { Гравитационное взаимодействие; } \\
\text { - лектромагнитное взаимодействие: } \\
\text { - } \quad \text { электрическое первичное } \\
\text { (элементарные точечные } \\
\text { электрические заряды); } \\
\text { - } \quad \text { магнитное вторичное } \\
\text { (элементарный магнитный заряд } \\
\text { отсутствует и присутствуют } \\
\text { только в виде диполя); }\end{array}$ & $\begin{array}{l}\text { - Антигравитационное } \\
\text { в Позимодействие; } \\
\text { взаимодействие: } \\
\text { - } \quad \text { позитронное (магнитное) } \\
\text { первичное (элементарные } \\
\text { топологические магнитные } \\
\text { заряды); } \\
\text { - } \text { электрическое вторичное } \\
\text { (элементарный электрический } \\
\text { заряд отсутствует и } \\
\text { присутствуют только в виде } \\
\text { диполя); }\end{array}$ \\
\hline \multicolumn{2}{|c|}{ - Идеальное обменное взаимодействие } \\
\hline \multicolumn{2}{|c|}{ - Аннигиляционное взаимодействие } \\
\hline \multicolumn{2}{|c|}{ - Слабое взаимодействие } \\
\hline $\begin{array}{l}\text { - } \quad \text { В TSSCU скалярное поле Браута-Энгле } \\
\text { фундаментальным. Гипотеза о происх } \\
\text { имеющего спин равный } 0\left({ }_{t} S=0\right), \text { а т } \\
\text { времени }{ }^{L} S T \text { и }{ }^{D} S T \text { будет дана в следу }\end{array}$ & $\begin{array}{l}\text { ра-Хиггса }(B E H) \text { - является } \\
\text { ождении этого поля и бозона Хиггса } H \text {, } \\
\text { Ікже его распределения в пространствах- } \\
\text { ощей статье «Формализм TSSCU» }\end{array}$ \\
\hline
\end{tabular}

В главе 5 уже рассматривалось электромагнитное и позитронноэлектрическое (сильное) взаимодействия, а в главе 6 гравитационное и антигравитационное. Эти 4 фундаментальных взаимодействия строго подразделяются по своему действию: гравитационное и электромагнитное относятся к ${ }^{L} S T$, а антигравитационное и позитронноэлектрическое (сильное) относятся к ${ }^{D} S T$.

Идеальное обменное, аннигиляционное и слабое взаимодействия в $T S S C U$ относятся к межпространственным, в которых оба пространства-времени ${ }^{L} S T$ и ${ }^{D} S T$, в той или иной степени, принимают непосредственное участие. В главе 7 уже рассматривалось идеальное обменное взаимодействие. Несмотря на впечатляющие успехи современной теория слабого взаимодействия $[113,114,115,116,117,118,119,120$, $121,122,123,124,125,126,127,128,129,130,131]$, с позиции TSSCU она является неполной. Рассматривать новые подходы к этой теории имеет смысл после понимания роли скалярного поля Браута-Энглера-Хиггса $(B E H)$ и бозона Хиггса $H$, имеющего спин равный $0\left({ }_{t} S=0\right)$, которые непосредственно принимают участие в слабом взаимодействии.

Остановимся на аннигиляционном взаимодействии, которое будет подробно рассмотрено в отдельной статье «Особенности калибровочной теории и СPT теоремы 
в TSSCU». Здесь же, кратко остановимся на некоторых моментах, имеющих непосредственное отношение к аннигиляции.

Как уже рассматривалось в п. 6.8 Главы 6 в ${ }^{D} S T$ действует антигравитация, которая расщепляет антиматерию. Расщепленная антиматерия, имеющая отрицательную массу и отрицательную энергию, принимает децентрализованные динамические формы, которым были даны названия топологические солитоны.

Гипотеза: $t$-солитоны антиматерии, при определенных условиях и обстоятельствах проникают из неоднородного и анизотропного пространства-времени ${ }^{D} S T$ в пространство-время ${ }^{L} S T$ и подчиняясь действию гравитации - преобразовываются в античастицы. При этом, в соответствии с особенностями CPT-теоремы в TSSCU (которая, будет подробнее рассмотрена в готовящейся публикации «Особенности калибровочной теории и CPT-теоремы в $T S S C U »)$, помимо уже прошедшей пространственной инверсии ( $P$ - инверсии), время ${ }^{D} T$ инвертируется на время ${ }^{L} T(T$ - инверсия), и в случае если $t$-солитон антиматерии обладает элементарным распределенным магнитным зарядом, он инвертируется в электрический заряд, противоположный электрическому заряду фундаментально-сцепленной с ним частицы в ${ }^{L} S T$ ( $C$ - инверсия). Таким образом, $t$-солитоны антиматерии трасформированные в античастицы, до встречи со своим фундаментальносцепленным партнером и последующей аннигиляции, полностью подчиняются законам, действующими в ${ }^{L} S T$. Собственно, именно это, на взгляд автора и подтверждают экспериментальные данные и исследования, проводимые различными коллаборациями $[132,133,134,135,136,137,138,139,140,141,142,143$, $144,145,146,147,148,149,150,151]$. Природа постаралась сделать все, чтобы скрыть прямые доказательства реальности существования пространства-времени ${ }^{D} S T$. 
Глава 10 Поля и преобладающие процессы в пространствах-времени ${ }^{L} S T$ и ${ }^{D} S T$

\begin{tabular}{|c|c|}
\hline Пространство-время ${ }^{L} S T$ & Пространство-время ${ }^{D} S T$ \\
\hline $\begin{array}{l}\text { • электрическое поле (первичное); } \\
\text { • } \quad \text { магнитное поле (вторичное) } \\
\text { Образуют: квази электромагнитное } \\
\text { поле с преобладающим процессом } \\
\text { линейного однородного расширения и } \\
\text { излучения электромагнитных волн } \\
\text { (ЭМВ). }\end{array}$ & $\begin{array}{l}\text { • } \quad \text { позитронное поле (магнитное } \\
\quad \text { первичное); } \\
\text { • } \quad \text { электрическое поле (вторичное) } \\
\text { Образуют: квази позитронно- } \\
\text { электрическое поле с преобладающим } \\
\text { процессом нелинейного неоднородного } \\
\text { сжатия и активного поглощения } \\
\text { (отрицательного излучения) } \\
\text { позитронно-электрических волн (ПЭВ). }\end{array}$ \\
\hline \multicolumn{2}{|c|}{ Пространство-время ${ }^{A} S T$} \\
\hline $\begin{array}{l}\text { В результате взаимодействия этих } \\
\text { идеального обменного взаимодейс } \\
\text { электромагнитное - позитронно-эл }\end{array}$ & $\begin{array}{l}\text { квазиполей, с учетом эффекта спина и } \\
\text { в }{ }^{A} S T \text { в } T_{\text {synch }} \text { образуется } \\
\text { оическое самосогласованное поле. }\end{array}$ \\
\hline
\end{tabular}


Глава 11 Принципы движения материальных тел в пространствахвремени ${ }^{L} S T$ и ${ }^{D} S T$

Рассмотрим движение фундаментально-сцепленных электрона (частицу) и позитрона ( $t$-солитона) в самосогласованном атоме в стационарном состоянии.

\begin{tabular}{|l|l|}
\hline \multicolumn{1}{|c|}{ Пространство-время ${ }^{L} S T$} & \multicolumn{1}{|l}{ Пространство-время ${ }^{D} S T$} \\
\hline $\begin{array}{l}\text { Пусть электрон (частица) в момент } \\
\begin{array}{l}\text { времени }{ }^{L} T \text { находится в точке } \\
\text { локализации } A .\end{array}\end{array}$ & $\begin{array}{l}\text { Пусть позитрон (частица) в момент } \\
\text { времени }{ }^{D} T \text { находится в точке } \\
\text { локализации } A .\end{array}$ \\
\end{tabular}

Спин ${ }_{t} S_{A}= \pm \frac{1}{2}$ в точке локализации $A-$ является «окном прозрачности» в $T_{\text {synch }}$ в ${ }^{A} S T$, при котором между электроном частицей и позитроном частицей (частицей, поскольку в ${ }^{A} S T$ реализуется геометрия Евклида с постоянной гравитацией ${ }^{A} G_{r}=1$ ) происходит «идеальное обменное взаимодействие», а времена ${ }^{L} T$ и ${ }^{D} T$ - синхронизируются.

- далее, в начальный момент времени ${ }^{L} T$, на электрон (частицу) действует положительная гравитация ${ }^{L} G_{r}$ и он незначительно трансформируется с идеальной окружности на овал;

- одновременно с этим электрон, используя полученный в момент ${ }_{t} S_{A}=\frac{1}{2}$ импульс и магнитный диполь (от позитрона), движется по часовой стрелке, по траектории отличной от окружности и стремящейся к эллиптической до точки локализации $D$, в соответствии с принципом наименьшего действия.
- далее, в начальный момент времени ${ }^{D} T$, на позитрон (частицу) действует отрицательная гравитация ${ }^{D} G_{r}$ и он путем гиперболического всплеска (резкое увеличение амплитуды с компенсаторным образованием дисперсии) без диссипации вынужденно трансформируется в $t$ - солитон;

- одновременно с этим позитрон, используя полученный в момент ${ }_{t} S_{A}=-\frac{1}{2}$ импульс и электрический диполь (от электрона), движется против часовой стрелки по гиперболическим $t$-солитонным траекториям до точки локализации $D$.

Спин ${ }_{t} S_{D}= \pm \frac{1}{2}$ в точке локализации $D$ - является «окном прозрачности» в $T_{\text {synch }}$ в ${ }^{A} S T$, при котором между электроном частицей и позитроном частицей (частицей, поскольку в ${ }^{A} S T$ реализуется геометрия Евклида с постоянной гравитацией ${ }^{A} G_{r}=1$ ), происходит «идеальное обменное взаимодействие», а времена ${ }^{L} T$ и ${ }^{D} T-$ синхронизируются. 
- далее, в начальный момент времени ${ }^{L} T$, на электрон (частицу) действует положительная гравитация ${ }^{L} G_{r}$ и он незначительно трансформируется с идеальной окружности на овал;

- одновременно с этим электрон, используя полученный в момент ${ }_{t} S_{D}=\frac{1}{2}$ импульс и магнитный диполь (от позитрона), движется по часовой стрелке, по траектории отличной от окружности и стремящейся к эллиптической до точки локализации $A$, в соответствии с принципом наименьшего действия.
- далее, в начальный момент времени ${ }^{D} T$, на позитрон (частицу) действует отрицательная гравитация и он путем гиперболического всплеска (резкое увеличение амплитуды с компенсаторным образованием дисперсии) без диссипации вынужденно трансформируется в $t$ - солитон;

- одновременно с этим позитрон, используя полученный в момент ${ }_{t} S_{D}=-\frac{1}{2}$ импульс и электрический диполь (от электрона), движется против часовой стрелки по гиперболическим $t$-солитонным траекториям до точки локализации $A$.

Несмотря на хаотические свойства ${ }^{D} S T$, позитрон ( $t$-солитон), будучи фундаментально-сцепленным с электроном (частицей), успешно справляется со своей задачей: начинать и заканчивать свое движение в точках локализации $A$ и $D$ (или $B$ и $C$ ). Помимо позитрона, другие $t$-солитоны антиматерии, включая темные фотоны, будучи фундаментально-сцепленными со своими партнерами в ${ }^{L} S T$, также двигаются согласованно, как единый хорошо управляемый организм. Необходимо учесть еще одно важное обстоятельство: зависимость от реализованной в пространстве-времени геометрии для $t$-солитонов антиматерии в ${ }^{D} S T$ значительно выше, чем для частиц материи в ${ }^{L} S T$. С учетом, множества изложенных в этой статье факторов мы предполагаем, что в движении $t$-солитона позитрона (и других фундаментально-сцепленных $t$-солитонов антиматерии) в ${ }^{D} S T$ должен соблюдаться какой-то новый принцип, эквивалентный принципу наименьшего действия для электрона (и других частиц) в ${ }^{L} S T$. Назовем его - принципом наибольшего действия, поскольку в пространстве-времени ${ }^{D} S T$ имеющем дробную размерность реализуется обратная зависимость (см. п. 3.7 Главы 3). Будем считать, что пакеты постоянно распадающихся и двигающихся в точку своей очередной локализации элементов $t$-солитонов антиматерии - действуют в соответствии с этим новым принципом. Если принять во внимание идею Р. Фейнмана [152] о том, что «позитрон» (интерпретация автора, с учетом выдвигаемых в настоящей статье положений; Фейнман, естественно рассуждал об электроне) проходит по всем возможным траекториям до точки своей локализации, то это является дополнительным подтверждением правильности нового принципа. При этом следует отметить, что оба принципа: наименьшего действия в ${ }^{L} S T$ и наибольшего действия в ${ }^{D} S T$ - необходимо рассматривать в неразрывной совокупности друг с другом. Фактически, каждый из них является главным доказательством «правильности» другого. 
Природа $t$-солитонов - первична в ${ }^{D} S T$, поэтому в нем реализуются как элементарные $t$-солитоны, так и группы (ансамбли) взаимодействующих $t$-солитонов. $\mathrm{B}{ }^{L} S T$ природа $t$-солитонов - вторична, поэтому в нем реализуются только вторичные группы (ансамбли) взаимодействующих $t$-солитонов. Такие и схожие процессы можно наблюдать в природе, даже на макроуровне. Например, мурмурации стаи птиц, движения роя пчел или стаи рыб [153], которые ждут своего математического решения.

Дальнейшее развитие теории движения $t$-солитонов антиматерии в ${ }^{D} S T$ возможны по двум путям. В первом случае, несмотря на уже имеющиеся весомые достижения, например, [154, 155, 156, 157, 158, 159], необходимо адаптировать существующие нелинейные дифференциальные уравнения, имеющие солитонные решения к реалиям ${ }^{D} S T$. Во втором случае, попытаться написать новые уравнения для ${ }^{D} S T$, учитывающие новые полученные знания и весь процесс: от начала образования $t$-солитона (гиперболического всплеска с образованием сети-кроны дерева) до его локализации (в точке на стволе дерева), в соответствии с принципом наибольшего действия. С учетом выводов, полученных в п. 3.9 Главы 3 о природе комплексных чисел, перспективный подход к решению проблемы по первому пути, частично реализован в работе [160].

Вывод 1: Электрон в точках локализации $A$ и $D$ (или $B$ и $C$ ) в ${ }^{A} S T$, а также во времени ${ }^{L} T$ неизменно представляет собой частицу и движется в ${ }^{L} S T$ в соответствии с принципом наименьшего действия. Позитрон в точках локализации $A$ и $D$ (или $B$ и $C$ ) ${ }^{A} S T$ представляет собой частицу (так как, там реализуется геометрия Евклида $(\mathcal{E}) \mathrm{c}$ постоянной гравитацией $\left.{ }^{A} G_{r}=1\right)$, а во времени ${ }^{D} T$ представляет собой $t$-солитон (s-облако) и движется в ${ }^{D} S T$ по гиперболическим $t$-солитонным траекториям, в соответствии с принципом наибольшего действия.

Вывод 2: Уравнение Шредингера описывает состояния системы фундаментальносцепленных электрона частицы в ${ }^{L} S T$ и $t$-солитона ( $s$-облака) позитрона в ${ }^{D} S T$. Этот удивительный вывод окажется более убедительным, если принять во внимание, что во временном уравнении Шредингера, время $\left({ }^{L} t\right)$ проявляется как производная первого порядка, а пространственные координаты $\left({ }^{D} S T\right.$ или проекции ${ }^{D} S T$ на $\left.{ }^{A} S T\right)$ выражаются как производные второго порядка. Таким образом, в уравнении Шредингера пространство и время - разнородны, то есть принадлежат разным пространствам-временам.

При этом комплексозначная волновая функция - является математическим инструментом, предоставляющим некоторую статистическую и иную информацию о движение $t$-солитона ( $s$-облака) позитрона по гиперболическим $t$-солитонным орбитам в ${ }^{D} S T$.

При проведении квантовых измерений инструмент исследователя (состоящий, как из частиц материи, так и из $t$-солитонов антиматерии) приходит во взаимодействие с элементом (или несколькими элементами) пакета $t$-солитона ( $s$-облака) позитрона в ${ }^{D} S T$. Наступает некоторая естественная мгновенная реакция (в $T_{\text {synch }}$ ) системы фундаментально-сцепленных электрона и позитрона. Как следствие этой реакции, волновая функция до этого момента, описывающая состояния системы, коллапсирует. 
В случае не стационарного (сильно возбужденного) состояния атома и различных резонансных явлений, нельзя исключить первичный гиперболический всплеск позитрона с возникновением «очень большой» амплитуды, и соответственно, ненулевой вероятности нахождения элементов $t$-солитона позитрона в любой области пространства-времени ${ }^{D} S T$.

Вывод 3: Уравнение Дирака можно интерпретировать как описывающее систему релятивистских фундаментально-сцепленных электрона частицы в ${ }^{L} S T$ c положительной массой и положительной энергией, с двумя точками локализации со спином ${ }_{t} S= \pm \frac{1}{2}$ в ${ }^{A} S T$ и позитрона $t$-солитона в ${ }^{D} S T$ с отрицательной массой и отрицательной энергией, с двумя точками локализации со спином ${ }_{t} S= \pm \frac{1}{2}{ }^{A} S T$.

Вывод 4: Принцип неопределенности Гейзенберга, действует только, если исследователь ограничивает себя рамками пространства-времени ${ }^{L} S T$. Действительно, в этом случае при нахождении точных координат электрона в ${ }^{L} S T$, его будущий импульс до момента очередной точки локализации - находится в пространстве-времени ${ }^{D} S T$ и информация о нем неопределенна. В случае, если исследователь выходит за рамки ${ }^{L} S T$ и проводит измерения в обоих пространствахвремени ${ }^{L} S T$ и ${ }^{D} S T$ - синхронно в $T_{\text {synch }}$ : состояния электрона в ${ }^{L} S T$ и состояния позитрона в ${ }^{D} S T$ с использованием всех 6 измерений (4 пространственных и 2 временных) для каждого пространства-времени, то он может получить любую требуемую информацию с абсолютной точностью.

Вывод 5: Строгое математическое определение $t$-солитона (заряженного и/или нейтрального) как формы антиматерии в пространстве-времени ${ }^{D} S T$ - является приоритетной задачей. Точная математическая формализация принципа наибольшего действия для движения $t$-солитонов антиматерии в пространствевремени ${ }^{D} S T$ в условиях неоднородности и анизотропности: и пространства и времени ${ }^{D} T$, а также нахождение уравнений движения - является исключительно важной задачей. Таким образом, нашей конечной целью - является нахождение системы двухкомпонентных самосогласованных уравнений движений в $T_{\text {synch }}$ : электрона (частицы) в ${ }^{L} S T$, в соответствии с принципом наименьшего действия, и позитрона ( $t$-солитона) в ${ }^{D} S T$, в соответствии с принципом наибольшего действия с использованием всех 6 измерений для каждого пространства-времени. 


\section{Глава 12 Заключение}

1. Вселенная - является единой и вечной. Вселенная включает в себя: первичное (материнское) Абсолютное пространство-время ${ }^{A} S T$ и Управляющую Супермнимую Единицу ${ }_{S} I$ которая периодически инициирует возникновение $B B$ с последующим неотвратимым $\mathrm{Cr}$.

2. Подход к определению размерности во Вселенной и ее архитектуры может заключаться в следующем. Исходя из нашей гипотезы и результатов, полученных в данной статье, мы можем предположить, что в период с $C r$ до $B B$ первичное (материнское) Абсолютное пространство-время ${ }^{A} S T$ находится в неопределенном состоянии, а Управляющая Супермнимая Единица ${ }_{s} I$ не взаимодействует с ${ }^{A} S T$ и находится в обособленном состоянии. В момент $B B$ и до $\mathrm{Cr}$ первичное (материнское) Абсолютное пространство-время ${ }^{A} S T$ (нулевой $(\Omega=0)$ и бесконечной $(\Omega=\infty)$ кривизны одновременно) взаимодействуя с мнимой параболической единицей I образуют обобщенное Паракомплексное Абсолютное пространство-время ${ }_{I}^{A} S T$, состоящего из 6 Паракомплексных измерений. В случае частичного расслоения этого обобщенного Паракомплексного Абсолютного пространства-времени ${ }_{I}^{A} S T$ они (измерения), троятся, образуя 18 измерений:

- 3 - пространственных координат ${ }^{A} S T$;

- 1 - времени ${ }^{A} T$;

- 1 - мнимой параболической пространственной единицы I;

- 1 -мнимого параболического времени ${ }_{I} T$;

- 3 - пространственных координат ${ }^{L} S T$;

- 1 - времени ${ }^{L} T$;

- 1 - мнимой эллиптической пространственной единицей $i$ в ${ }^{L} S T$;

- 1 - мнимого эллиптического времени - ${ }_{i} t$ в ${ }^{L} S T$;

- 3 - пространственных координат ${ }^{D} S T$;

- 1 - темного времени ${ }^{D} T$;

- 1 - темной мнимой гиперболической пространственной единицей $\tilde{\imath}$ в ${ }^{D} S T$;

- 1 - темного мнимого гиперболического времени - ${ }_{i} t$ в $^{D} S T$;

Таким образом, с учетом приоритетной для автора гипотезы о частичном расслоении обобщенного Паракомплексного Абсолютного пространства-времени ${ }_{I}^{A} S T$, на современном этапе во Вселенной реализуется - 12 рациональных и 6 мнимых измерений или:

- 4 пространственных и 2 временных Паракомплексных измерений в ${ }_{I}^{A} S T$;

- 4 пространственных и 2 временных комплексных измерений в ${ }_{i}^{L} S T$;

- 4 пространственных и 2 временных темных комплексных измерений в ${ }_{\tilde{\imath}}^{D} S T$;

3. На современном этапе во Вселенной относительно обобщенного Паракомплексного Абсолютного пространства - времени ${ }_{I}^{A} S T$ реализуется пространственно - временная симметрия. В момент $B B$ синхронно в $T_{\text {synch }}$ родились (образовались) два взаимопрозрачных пространства-времени ${ }^{L} S T$ и ${ }^{D} S T$. Ткань пространства-времени ${ }^{L} S T$ однородно расширяется (относительно неподвижной ткани ${ }^{A} S T$ ) за счет неоднородного сжатия ткани пространства- 
времени ${ }^{D} S T$. Справедливо и обратное, ткань пространства-времени ${ }^{D} S T$ неоднородно сжимается (относительно неподвижной ткани ${ }^{A} S T$ ) за счет однородного расширения ткани пространства-времени ${ }^{L} S T$.

4. Предложенные алгоритмы расслоения первичного (материнского) Абсолютного пространства-времени ${ }^{A} S T$ с геометрией Евклида $(\mathcal{E})$, в виде уравнений 5-23 является предварительными и неполными. Они нуждаются в расширении за счет:

- включения в них трех мнимых единиц: параболической $I$, эллиптической $i$, гиперболической $\tilde{l}$, каждой из которых соответствует свое пространствовремя;

- определения и включения в них кванта расширения пространства-времени ${ }^{L} S T$, самосогласованного с темными квантами сжатия пространства-времени ${ }^{D} S T$;

- $\quad$ изучения природы и свойств времени синхронизации $T_{\text {synch }}$;

5. Судьба образовавшихся синхронно в $T_{\text {synch }}$ в обобщенном Паракомплексном Абсолютном пространстве - времени ${ }_{I}^{A} S T$ в момент $B B$ двух пространств-времен ${ }^{L} S T$ и ${ }^{D} S T$ предрешена: в будущем они исчезнут в момент $C r$, также синхронно в $T_{\text {synch }}$.

6. Барионная асимметрия во Вселенной - отсутствует. С момента $B B$ вся рожденная материя развивается, в наблюдаемом нами, пространстве-времени положительной кривизны ${ }^{L} S T$, а вся рожденная антиматерия развивается, в не наблюдаемом нами, пространстве-времени отрицательной кривизны ${ }^{D} S T$. Та часть антиматерии, которая по определенным причинам (будут рассмотрены нами в одной из последующих статей «Особенности калибровочной теории и СPTтеоремы в $T S S C U »)$, проникает в пространство-время ${ }^{L} S T$ - аннигилирует с материей, сохраняя паритет.

7. Антиматерия с отрицательной плотностью материи и отрицательной плотностью энергии, образовавшуюся в момент $B B$ и развивающуюся в пространстве-времени ${ }^{D} S T$ можно также называть темной материей (или скрытой материей) обладающей темной энергией (скрытой энергией). В соответствии, с частичным расслоением ${ }_{I}^{A} S T$ основную плотность во Вселенной - составляют: первичная управляющая материя ${ }^{A} M$ с первичной управляющей энергией ${ }^{A} E$.

8. Рождение в момент $B B$ двух взаимопрозрачных и, одновременно, самосогласованных пространств-времен ${ }^{L} S T$ и ${ }^{D} S T$ со своими временами ${ }^{L} T$ и ${ }^{D} T$ вынуждает нас ввести понятие времени синхронизации $T_{\text {synch }}$. Одно из важнейших свойств $T_{\text {synch }}$ - заключается в том, что образование парных объектов в пространствах-времени ${ }^{L} S T$ и ${ }^{D} S T$ синхронно в $T_{\text {synch }}$ наделяет эти объекты свойством фундаментальной-сцепленности æ.

9. Реализация «эффекта самосогласованности» во Вселенной стала возможной, благодаря новому способу введения обобщенных комплексных чисел. Каждая мнимая единица: параболическая $I$, эллиптическая $i$ и гиперболическая $\tilde{l}$, задается в строгом соответствии со знаком кривизны своего пространства-времени. Именно, обобщенные комплексные числа, развивающие теории функций 
комплексных переменных, играют главную роль в обеспечении межпространственно-временной коммуникации.

10. Природа массы: частицы материи с массой ${ }^{L} m$ и $t$-солитоны антиматерии с массой ${ }^{D} m$ рождаются парами синхронно в $T_{\text {synch }}$ из ячеек тканей пространств-времен ${ }^{L} S T$ и ${ }^{D} S T$, соответственно. При этом частицы с массой ${ }^{L} m$ и $t$-солитоны с массой ${ }^{D} m-$ будут обладать свойством фундаментальной сцепленности. Исключением из этого правила является масса бозона Хиггса $H$, имеющего спин равный $0\left({ }_{t} S=0\right)$, который в $T S S C U$ играет свою фундаментальную роль.

11.Понимание трех пространственно-временной природы спина ${ }_{t} S$ (окно прозрачности в ${ }^{A} S T$ ) позволяет нам не только формализовать «идеальное обменное взаимодействие» для фермионов и бозонов, но и сделать точные безальтернативные предсказания:

- частиц, которые являются античастицами сами для себя - не существует;

- безмассовый нейтральный бозон фотон - $\gamma$ в ${ }^{L} S T$, имеющий спин ${ }_{t} S= \pm 1$ обязан иметь партнера безмассового нейтрального темного фотона - ${ }_{\text {в }}{ }^{D} S T$, также со спином ${ }_{t} S= \pm 1$;

- массивный бозон ${ }^{0} Z$ в ${ }^{L} S T$, имеющий спин ${ }_{t} S= \pm 1$ - обязан иметь партнера нейтрального массивного бозона из антиматерии ${ }^{0} \tilde{Z}$ в ${ }^{D} S T$, также со спином ${ }_{t} S= \pm 1$.

12. Принципы наименьшего действия для частиц материи в ${ }^{L} S T$ и наибольшего действия для $t$-солитонов антиматерии в ${ }^{D} S T$, их фундаментальная-сцепленность и самосогласованность в движении открывает широкие возможности для построения точных математических моделей движения в пространствах-времени ${ }^{L} S T$ и ${ }^{D} S T$.

13. Понятие фундаментальной-сцепленности, принципы наименьшего и наибольшего действий, «идеальное обменное взаимодействие», $t$-солитоны как форма антиматерии в ${ }^{D} S T$, а также гипотеза о том, что позитрон в ${ }^{D} S T$ обладает элементарным топологическим магнитным зарядом, подводят к заключительному этапу построения теории самосогласованного частицесолитонного атома.

14. Сильное ядерное взаимодействие, применяемое сегодня в физике, в TSSCU представляет собою позитронно-электрическое взаимодействие в пространствевремени ${ }^{D} S T$. При этом, позитрон и антипротон (а также другие зарядовые $t$ солитоны) в ${ }^{D} S T$ имеют элементарный топологический магнитный заряд $\tilde{\theta}$, количественное значение которого предстоит определить. Помимо этого, в пространстве-времени ${ }^{D} S T$ должны быть свои темные кванты действия ${ }^{D} h$ и ${ }^{D} \hbar_{1-n}$ эквивалентные постоянной Планка ${ }^{L} h$ и приведенной постоянной Планка ${ }^{L} \hbar$. Определение величин элементарного топологического магнитного заряда $\tilde{\theta}$, а также темных квантов действия (активного поглощения или притяжения) ${ }^{D} h$ и ${ }^{D} \hbar_{1-n}-$ позволит, в обозримом будущем, создать полноценную теорию сильного взаимодействия, в виде системы уравнений для ${ }^{D} S T$, эквивалентных модифицированным уравнениям Максвелла для ${ }^{L} S T$. 
15. Для решения проблемы квантования гравитации, с учётом трех пространственновременной модели строения Вселенной, следует поставить вопрос по-новому: необходимо определить квант расширения (в паре: расстояния-радиуса кривизны - ${ }^{L} S$ и кривизны - $\left.{ }^{L} \omega\right)$ в ${ }^{L} S T$, а также темные кванты сжатия (в паре: расстояниярадиуса кривизны - ${ }^{D} S$ и кривизны - $\left.{ }^{D} \omega\right)$ в ${ }^{D} S T$. Для этого необходимо сформулировать и найти решения системы самосогласованных уравнений, описывающих действие гравитации в ${ }^{L} S T$ и антигравитации в ${ }^{D} S T$, с учетом топологического вращательного механизма стабилизации.

Помимо этого, несмотря на динамические хаотические свойства пространствавремени ${ }^{D} S T$, необходимо попытаться найти подходы к написанию уравнений к закону (нам) всемирного антитяготения в ${ }^{D} S T$ эквивалентному закону всемирного тяготения в ${ }^{L} S T$.

16. Гипотеза о том, что термоядерные реакции являются основным источником энергии во Вселенной, как минимум, не полна.

Все многообразие существующих материальных и антиматериальных миров в трех пространственно-временной Вселенной можно объяснить за счет взаимопрозрачности пространств-времен ${ }^{L} S T$ и ${ }^{D} S T$, их фундаментальной сцепленности и взаимного антагонизма. 


\section{Использованные термины, обозначения и сокращения}

${ }^{A} S T$ - первичное (материнское) Абсолютное пространство-время (Absolut SpaceTime);

$s^{I}$ - Управляющая Супермнимая Единица;

$I$ - мнимая параболическая единица в ${ }^{A} S T$;

${ }_{I}^{A} T$ - мнимое параболическое время в ${ }^{A} S T$;

${ }_{I}^{A} S T$ - Обобщенное Паракомплексное Абсолютное пространство-время;

${ }^{A} M-$ первичная материя в ${ }^{A} S T$;

${ }^{A} E$ - первичная энергия в ${ }^{A} S T$;

${ }^{A} I$ - первичная информация в ${ }^{A} S T$;

${ }^{A} m$ - масса частицы в ${ }^{A} S T$;

${ }^{A} S$ - расстояние в ${ }^{A} S T$;

${ }^{A} \Omega$ - кривизна в ${ }^{A} S T$;

${ }^{A} T$ - время в ${ }^{A} S T$;

${ }^{A} V$ - скорость в ${ }^{A} S T$;

${ }^{A} G_{r}$ - гравитация в ${ }^{A} S T$;

${ }^{A} K$ - температура в ${ }^{A} S T$;

$B B$ - Большой Взрыв;

$\mathrm{Cr}$ - Схлопывание;

${ }^{S T} U$ - межпространственный потенциал;

$T_{\text {synch }}$ - время синхронизации;

${ }_{t} S$ - спин;

$(\varepsilon)$ - геометрия Евклида;

$\bar{æ}$ - фундаментальная сцепленность между тканями пространств-времен ${ }^{L} S T$ и ${ }^{D} S T$, а также частицами в ${ }^{L} S T$ и их партнерами $t$-солитонами в ${ }^{D} S T$;

${ }^{L} S T$ - пространство-время (Light (Real) Space-Time);

$i$ - мнимая эллиптическая единица в ${ }^{L} S T$;

${ }_{i} t$ - мнимое эллиптическое время в ${ }^{L} S T$;

${ }_{i}^{L} S T$ - эллиптическое комплексное пространство-время;

${ }^{L} M$ - материя (с положительной плотностью) в ${ }^{L} S T$;

${ }^{L} E$ - энергия (с положительной плотностью) в ${ }^{L} S T$; 
${ }^{L} I$ - информация стохастическая в ${ }^{L} S T$;

${ }^{L} m$ - масса частицы в ${ }^{L} S T$;

${ }^{L} S$ - расстояние в ${ }^{L} S T$;

${ }^{L} \omega-$ кривизна в ${ }^{L} S T$;

${ }^{L} t-$ время в ${ }^{L} S T$;

${ }^{L} v$ - скорость в ${ }^{L} S T$;

${ }^{L} G_{r}-$ гравитация в ${ }^{L} S T$;

${ }^{L} K$ - температура в ${ }^{L} S T$;

$c$ - скорость света в ${ }^{L} S T$;

${ }^{L} \gamma$ или $\gamma$ - фотон в ${ }^{L} S T$;

$e^{-}$- электрон;

$\dot{e}^{+}-$позитрон в ${ }^{L} S T$;

$q$ - элементарный точечный электрический заряд в ${ }^{L} S T$;

$\theta^{+-}$- дипольные магнитные заряды в ${ }^{L} S T$;

${ }^{l} h$ - постоянная Планка в ${ }^{L} S T$;

$l^{l} \hbar$ - приведенная постоянная Планка в ${ }^{L} S T$;

$l_{p}$ - Планковская длина в ${ }^{L} S T$;

${ }^{L} G$ - гравитационная постоянная в ${ }^{L} S T$;

${ }^{L} H_{b}$ - динамический параметр Хаббла в ${ }^{L} S T$;

GR (ОТО) - Общая Теория Относительности;

$L Q G$ - Петлевая Квантовая Гравитации;

$(\mathcal{R})$ - геометрия Римана;

${ }^{D} S T$ - пространство-время (Dark (Hidden) Space-Time);

$\tilde{\imath}$ - мнимая гиперболическая единица в ${ }^{D} S T$;

${ }_{i} t-$ темное мнимое гиперболическое время в ${ }^{D} S T$;

${ }_{\tilde{\imath}}^{D} S T$ - гиперболическое темное комплексное пространство-время

${ }^{D} M$ - темная материя (антиматерия с отрицательной плотностью) в ${ }^{D} S T$;

${ }^{D} E$ - темная энергия (с отрицательной плотностью энергии) в ${ }^{D} S T$;

${ }^{D} I$ - информация фрактальная в ${ }^{D} S T$;

${ }^{\mathrm{D}} m$ - масса $t$-солитона в ${ }^{D} S T$; 
${ }^{D} S$ - расстояние в ${ }^{D} S T$;

${ }^{D} \omega-$ кривизна в ${ }^{D} S T$;

${ }^{D} t-$ время в ${ }^{D} S T$;

${ }^{D} v$ - скорость в ${ }^{D} S T$;

${ }^{D} G_{r}$ - антигравитация в ${ }^{D} S T$;

${ }^{D} K$ - температура в ${ }^{D} S T$;

${ }^{D} \gamma$ или $\tilde{\gamma}$ - темный фотон в ${ }^{D} S T$ (глюон - $g$, магнон, также являются ${ }^{D} \gamma$ );

$\tilde{c}$ - скорость темного света в ${ }^{D} S T$ для алгебры с доминантным минусом;

$\frac{1}{\tilde{c}}$ - скорость темного света в ${ }^{D} S T$ для алгебры с абсолютным доминантным минусом;

$\widetilde{e}^{+}-$позитрон в ${ }^{D} S T$;

$\tilde{\theta}$ - элементарный топологический магнитный заряд в ${ }^{D} S T$;

$\tilde{q}^{-+}$- дипольные топологические электрические заряды в ${ }^{D} S T$;

${ }^{d} h$ - темный флуктуационный квант действия в ${ }^{D} S T$ эквивалентный постоянной Планка ${ }^{l} h$ в ${ }^{L} S T$;

${ }^{d} \hbar_{1-n}$ - темные флуктуационные кванты действия в ${ }^{D} S T$ эквивалентные приведенной постоянной Планка ${ }^{1} \hbar$ в ${ }^{L} S T$;

${ }^{D} G_{1-n}$ - антигравитационные флуктуационные параметры в ${ }^{D} S T$;

${ }^{D} H_{b_{1-n}}$ - динамические флуктуационные параметры в ${ }^{D} S T$ эквивалентные параметру Хаббла в ${ }^{L} S T$;

SNT - Теория Струнных Сетей;

$(\mathcal{L B})$ - геометрия Лобачевского-Больяи;

$B E H$ - скалярное поле Браута-Энглера-Хиггса;

$H$ - Бозон Хиггса в ${ }^{D} S T$;

$S T$ - Теория струн;

CST - Теория Управляющих Струн. 


\section{Список литературы}

[1] A. Einstein, Die Feldgleichungen der Gravitation, Sitzungsberichte der Preuss. Akad. der Wissenschaften, Berlin, (1915), P. 844-847.

[2] D. Hilbert, «Die Grundlagen der Physic», Nachrichten Kon. Gesellshaft Wiss. Gottingen, Math. - Phys. Klasse, Heft 3, (1915), P. 395-407.

[3] Albert Einstein, " Cosmological Considerations in the General Theory of Relativity " Sitzungsberichte der Preuss. Akad. der Wissenschaften, Berlin, (1917), P. 142-152.

[4] W. de Sitter, On Einstein's Theory of Gravitation and its Astronomical Consequences, Monthly Notices of the Royal Astronomical Society, Vol. 76, Iss. 9, (1916), P. 699-728.

[5] Friedmann, A: «Über die Krümmung des Raumes», Zeitschrift für Physik, Vol. 10, (1922), P. 377-386.

[6] Friedmann, A: «Über die Möglichkeit einer Welt mit konstanter negativer Krümmung des Raumes», Zeitschrift für Physik, Vol. 21, (1924), P. 326-332.

[7] Kaluza T. "Zum Unitätsproblem der Physic”, Sitzungsberichte der Preuss. Akad. der Wissenschaften, Berlin, Math. - Phys., (1921), P. 966-972.

[8] Klein 0., Quantentheorie und fünfdimensionale Relativitätstheorie, Zeitschrift für Physik, Vol. 37, (1926), P. 895-906.

[9] Lemaître, G. "A Homogeneous Universe of Constant Mass and Growing Radius, Accounting for the Radial Velocity of the Extragalactic Nebulae", Annales de la Société Scientifique de Bruxelles 47, (1927), P. 49-59.

[10] Vesto M. Slipher, Nebulae., Proc. Am. Philos. Soc., Vol. 56, (1917), P. 403-409.

[11] Howard P. Robertson, "On Relativistic Cosmology”, Philos. Mag. and Journal of Science, Vol. 5, (1928), P. 835-848.

[12] Edwin P. Hubble, A Relation between distance and radial velocity among extra-galactic nebulae, Proc. Nat. Acad. Sci., 15 (3), (1929), P. 168-173.

[13] Ralph A. Alpher and Robert Herman, Evolution of the Universe, Nature, 162, (1948), P. 774-775.

[14] Dicke R. H., Peebles P. J. E., Roll P. G., Wilkinson D. T., Cosmic Black-Body Radiation., The Astrophysical Journal, Vol. 142, (1965), P. 414-419.

[15] Penzias A. A., Wilson R. W., A Measurement of Excess Antenna Temperature at 4080 Mc/s., The Astrophysical Journal, Vol. 142, (1965), P. 419-421.

[16] F. Hoyle, A New Model for the Expanding Universe, Monthly Notices of the Royal Astronomical Society, Vol. 108, Iss. 5, (1948), P. 372-382.

[17] H. Bondi, T. Gold, The Steady-State Theory of the Expanding Universe, Monthly Notices of the Royal Astronomical Society, Vol. 108, Iss. 3, (1948), P. 252-270. 
[18] Gustav Naan, "Symmetrical Universe”, Publications of Tartu Astronomical observatory, Vol. XXXIV, (1964), P. 423-471.

[19] W. Tiller, "The Positive and Negative Space-Time Frames as Conjugate Systems", (Stanford, Preprint (1975), in Future Science, ed. White and Krippner (Garden City, NY: Doubleday \& Co., Inc.), (1977), P. 257-279.

[20] John A. Wheeler, Genesis and Observership, Foundational Problems in the Special Sciences., Ed. by R. E. Butt, J. Hintikka, Dordrecht: D. Reidel, (1977), P. 3-33.

[21] Barrow J. D., Tipler F. J., The Anthropic Cosmological Principle., Clarendon, Oxford University Press, (1986).

[22] David Bohm, "Hidden Variables and the Implicate Order", Zygon 20 (2), (1985), P. 111-124.

[23] Alain Connes, Noncommutative Geometry, (InterEditions Paris (1990)) San Diego: Academic Press, (1994), 654 p.

[24] Strukov I. A., Brukhanov A. A., Skulachev D. P. Sazhin M. V., Anisotropy of the microwave background radiation, Pis'ma v Astronomicheskii Zhurnal (Astronomy Letters), Vol. 18, N. 5, (1992), P. 387-395.

[25] George F. Smoot et al. "Structure in the COBE differential microwave radiometer first year maps”, Ap. J. Lett., 396, (1992), L1.

[26] Adam G. Riess, et al., Observational Evidence from Supernovae for an Accelerating Universe and Cosmological Constant, Astronomical Journal, Vol. 116, (1998), P. 1009-1038.

[27] S. Perlmutter, et al., Measurements of Omega and Lambda from 42 High-Redshift Supernovae, Astrophysical Journal, Vol. 517, (1999), P. 565-586.

[28] Yu. S. Vladimirov, The Nature of Space and Time: An Anthology of Ideas, Moscow: Lenand, (2015), $400 \mathrm{p}$.

[29] Schwartz M. D., Quantum Field Theory and Standard Model, Cambridge University Press, (2013), 952 p.

[30] V. L. Ginzburg, "What problems of physics and astrophysics seem now to be especially important and interesting?", UFN, Vol. 169, N. 4, (1999), P. 419-441.

[31] I. F. Ginzburg, Unsolved problems in fundamental physics, UFN, Vol. 179, N. 5, (2009), P. 525-529.

[32] John C. Baez. Open Questions in Physics, (2012).

http://math.ucr.edu/home/baez/physics

[33] S. Weinberg, Lectures in Particles and Field Theory, ed. S. Deser and K. Ford, Phys. Lett. 9, (1964), p. 357; Phys. Rev. 8138, (1965), p. 988.

[34] A. D. Sakharov, Violation of CP Invariance, C asymmetry, and baryon asymmetry of the universe, JETP Lett., Vol. 5, (1967), P. 32-35. 
[35] Tolman R. C. On the Problem of the Entropy of the Universe as a Whole., Phys. Rev., 37, (1931), P. 1639-1660.

[36] Tolman R. C. On the Theoretical Requirements for a Periodic Behaviour of the Universe., Phys. Rev., 38, (1931), P. 1758-1771.

[37] Starobinsky A. A. Spectrum of relict gravitational radiation and the early state of Universe. JETP Lett., Vol. 30, (1979), P. 682-685.

[38] Starobinsky A. A. A new type of isotropic cosmological model without singularity. Phys. Lett. B, Vol. 91, (1980), P. 99-102.

[39] Guth A. H. Inflationary Universe: A possible solution to the horizon and flatness problems. Phys. Rev. D, Vol. 23, (1981), P. 347-356.

[40] Mukhanov V. F., Chibisov G. V. Quantum Fluctuation and "Nonsingular Universe”. JETP Lett., Vol. 33, (1981), P. 532-535.

[41] Linde A. D. A New Inflationary Universe Scenario: A Possible Solutions of the Horizon, Flatness, Homogeneity, Isotropy and Primordial Monopole Problems. Phys. Lett. B, Vol. 108, (1982), P. 389-393.

[42] Albrecht A., Steinhardt P. J., Cosmology for Grand Unified Theories with Radiatively Induced Symmetry Breaking. Phys. Rev. Lett., Vol. 48, (1982), P. 1220-1223.

[43] A.D. Linde, "Inflationary Cosmology", Lecture Notes in Physics, 738, (2008), P. 1-54.

[44] A. Ijjas, P. J. Steinhardt, A. Loeb, “Inflationary paradigm in trouble after Planck 2013", Phys. Lett. B. 723, (2013), P. 261-266.

[45] Guth A. H., Kaiser, D. I. and Nomura, Y., "Inflationary Paradigm after Planck 2013," Physics Letters B. 733, (2014), P. 112-119.

[46] A. Linde, “Inflationary Cosmology after Planck 2013”, arXiv:1402.0526, (2014), P. 79.

[47] A. Ijjas, P. J. Steinhardt, A. Loeb, "Inflationary schism”, Phys. Lett. B. 736, (2014), P. 142-146.

[48] A. D. Sakharov, "The initial stage of an expanding Universe and the appearance of a non-uniform distribution of Matter", JETP Letter, Vol. 49, (1965), P. 345-358.

[49] Ia. B. Zeldovich, "The "hot" model of the Universe", UFN, Vol. 89, (1966), P. 647-668.

[50] Ia. B. Zeldovich, I. D. Novikov, «Structure and evolution of the Universe”, Moscow: Izdatel'stvo Nauka, (1975), 736 p., P. 657-663.

[51] Einstein A., Zum kosmologischen Problem der allgemeinen Relativitätstheorie, Sitzungsberichte der Preuss. Akad. der Wissenschaften, Berlin, (1931), P. 235-237.

[52] A. D. Sakharov, "Cosmological Models of the Universe with Rotation of Time's Arrow", JETP Letter, Vol. 79, Iss. 3(9), (1980), P. 689-693. 
[53] A. D. Sakharov, «Multi-sheeted models of the universe», JETP Letter, Vol. 83, (1982), P. 1233-1240.

[54] A. D. Sakharov, "Cosmological transitions with change of the sign of metrics”, JETP Letter, Vol. 87, (1984), P. 375-383.

[55] P. J. Steinhardt, Neil Turok, A Cyclic Model of the Universe, Science, V. 296, (2002), P. 1436-1439.

[56] Lauris Baum, Paul H. Frampton, Turnaround in Cyclic Cosmology, Phys. Rev. Lett., Vol. 98, N. 7, (2007), 071301.

[57] Latham Boyle, Kieran Finn, Neil Turok «CPT-Symmetric Universe», Phys. Rev. Lett., 121(25), (2018), 251301(5).

[58] J. S. Farnes «A unifying theory of dark energy and dark matter: Negative masses and matter creations within a modified $\Lambda$ CDM framework», A\&A, 620, A92, (2018).

[59] Einstein A., Podolsky B., Rosen N., «Can Quantum-Mechanical Description of Physical Reality Be Considered Complete? », Phys. Rev., Vol. 47, Iss. 10, (1935), P. 777-780;

[60] Feynman R. The theory of positrons. Phys. Rev., Vol. 76, Iss. 6, (1949), P. 749-759.

[61] H. Poincaré, "The Measure of Time”, (Revue de métaphysique et de morale, 6: 1-13, 1898), The Foundations of Science, N.-Y.: Science Press, (1913), P. 222 - 234.

[62] H. A. Lorentz "Electromagnetic phenomena in a system moving with any velocity smaller than that of light", KNAW (Amsterdam), Proceedings, 6, (1904), P. 809-813.

[63] A. Einstein, "Zur Elektrodynamic der bewegter Körper”, Ann. Physik, 17, (1905), P. 891-921.

[64] H. Poincaré "Sur la dynamique de l'électron", Rendiconti del Circolo matematico di Palermo, 21, (1906), P. 129-176.

[65] A. Einstein, "Einfluss der Schwerkraft auf die Ausbreitung des Lichtes", Ann. Physik, 35, (1911), P. 898-908.

[66] A. Einstein, “Die Grundlage der allgemeinen Relativitatstheorie”., Ann. Physik, Vol. 49, (1916), P. $769-822$.

[67] Robinson A. Non-standard analysis. -Princeton: Princeton Univ. Press, (1996), 293 p.

[68] Kanovei V. and Reeken M. Nonstandard analysis. Axiomatically. Berlin: SpringerVerlag, (2004), XVI+408 p.

[69] Gordon E. I., Kusraev A. G. Kutateladze S. S., Infinitesimal Analysis., Dordrecht etc.: Kluwer Academic Publishers, (2002), XIV+422 p.

[70] Yaglom I. M. "Complex Number and its Application in Geometry", Moscow: Fizmatgiz, (1963), 192 p., P. 13-19. // Complex Numbers in Geometry, Academic Press, (1968), 256 p.

[71] William K. Clifford, On the Space Theory of Matter, Proc. of the Cambridge Phil. Soc., 2, (1876), P. 157-158. 
[72] D. Hestenes, G. Sobczyk, Clifford Algebra to Geometric Calculus - A Unified Language for Mathematical and Physics, Reidel Publishing Company, (1984), 336 p.

[73] Lavrentiev M. A., Shabat B. V., Problems of hydrodynamics and their mathematical models, Moscow: Science, (1973). 416 p., P. 50-56.

[74] Frobenius G., “Über das Pfaffische Probleme”, Jl. für die reine u. angew. Math. 82, (1877), P. 230-315.

[75] Max Tegmark: “The Mathematical Universe”, Found. Phys. 38, (2008), P. 101-150;

[76] V. S. Vladimirov, I. V. Volovich, Ye. I. Zelenov «p-Adic Analysis and Mathematical Physics». Singapore: World Scientific, (1994), 352 p.

[77] B. Dragivich, A. Yu. Khrennikov, S. V. Kosyrev, I. V. Volovich, E. I. Zelenov. p-Adic Mathematical Physics: The First 30 Years. p-Adic Numbers, Ultrametric Anal. Appl., 9:2 (2017), P. 87-121.

[78] J. Schwinger «A Magnetic Model of Matter», Science, Vol. 165 (N. 3895), (1969), P. 757-761.

[79] Mandelbrot B. B., The Fractal Geometry of Nature, San Francisco: W. H. Freeman and Co., (1982), 460 p.

[80] Robert L. Oldershaw. "Self-Similar Cosmological Model: Introduction and Empirical Tests" , International Journal of Theoretical Physics, Vol. 28, No. 6, (1989), P. 669-694.

[81] Y. Baryshev, P. Teerikorpi. Discovery of Cosmic Fractals, World Scientific Publishing Co. Pte. Ltd., (2002), 363 p.

[82] G. M. Zaslavsky, R. Z. Sagdeev, D. A. Usikov, A. A. Chernikov, Weak Chaos and QuasiRegular Patterns., Cambridge: Cambridge University Press, (1991), 265 p.

[83] Kuznetsov S. P., Hyperbolic Chaos: A Physicist's View, Higher Education Press: Beijing and Springer-Verlag: Berlin, Heidelberg, (2012), 336 p.

[84] V. S. Vladimirov, I. V. Volovich, "Superanalysis. I. Differential Calculus”, TMF, Vol. 59, N. 1, (1984), P. 3-27.

[85] I. V. Volovich, "p-adic Space-Time and String Theory", TMF, Vol. 71, N. 3, (1987), P. 337-340.

[86] Alexander G. Parkhomov. Space. Earth. Human.: New Views on Science., Moscow: Izdatel'stvo Nauka, (2009), 272 p., P. 20-38. // Independently published, (2019), 251 p.

[87] Gorban A. N., Kegl B., Wunsch D., Zinovyev A., Principal Manifolds for Data Visualization and Dimension Reduction., Vol. 58, Springer, Berlin-Heidelberg-New York, (2008), $340 \mathrm{p}$.

[88] Gorban A. N., Zinovyev A., Principal Graphs and Manifolds. In Handbook of Research on Machine Learning Applications and Trends: Algorithms, Methods and Techniques., (eds. 
Olivas E. S., Guererro J. D. M., Sober M. M., Benedito J. R. M., Lopes A. J. S.). Information Science References, Hershey, PA, (2009).

[89] Gorban A. N., Zinovyev A., Principal Manifolds and Graphs in Practice: from Molecular Biology to Dynamical Systems., Int. Journ. Neural Syst., 20, (2010), P. 219-232.

[90] Dirac P. A. M., Quantized singularities in the electromagnetic field, Proc. Roy. Soc. Ser. A, Vol. 133, Iss. 821, (1931), P. 60-72.

[91] Gerard 't Hooft, "Magnetic monopoles in unified gauge theories", Nuclear Physics B. 79 (2), (1974), P. 276-284.

[92] Polyakov A.M., "Particle spectrum in quantum field theory", Journal of Experimental and Theoretical Physics Letters, Vol. 20, (1974), P. 194.

[93] Coleman S., The Magnetic Monopole Fifty Years Later, UFN, Vol. 144, (1984), P. $277-$ 340.

[94] Ade P. A. R. et al. (Planck Collaboration). Planck 2013 results. I. Overview of products and scientific results, Astronomy \& Astrophysics, Vol. 571, A1, (2014), P. 48.

[95] Aghanim N. et al. (Planck Collaboration), "Planck intermediate results. XLVI. Reduction of large-scale systematic effects in HFI polarization maps and estimation of the reionization optical depth", Astronomy \& Astrophysics, Vol. 596, A107, (2016), P. 52.

[96] Adam G. Riess, et al., "A 2.4\% Determination of the Local Value of the Hubble Constant", The Astrophysical Journal, Vol. 826, Issue 1, (2016), P. 33.

[97] Adam G. Riess, Stefano Casertano, Wenlong Yuan, Lucas M. Macri, and Dan Scolnic, "Large Magellanic Cloud Cepheid Standards Provide a 1\% Foundation for the Determination of the Hubble Constant and Stronger Evidence for Physics beyond $\Lambda$ CDM.", The Astrophysical Journal, Vol. 876, Issue 1, (2019), P. 85.

[98] Blokhintsev D. I., Space and time in the microworld, Moscow: Science, (1982), 347 p., P. 256-283.

[99] Carlo Rovelli, (2001). Quantum space-time: what we do know? (In C. Calender and N. Hugget (eds.), "Physics Meets Philosophy at the Planck scale", Cambridge University Press, P. 101-122.

[100] Terletsky, Ya. P., "Hypothesis of birth of particle pair with positive and negative masses from vacuum and its cosmological consequence", in the book "Problems of theoretical Physics", Moscow: publishing house UDN, (1990), P. 3-7.

[101] Abhay Ashtekar, "New Variables for Classical and Quantum Gravity”, Phys. Rev. Lett., 57(18), (1986), P. 2244-2247.

[102] Abhay Ashtekar, Jorge Pullin (eds.). Loop Quantum Gravity: The First 30 Years., (In 100 Years of General Relativity), World Scientific Publishing, (2017), 320 pp.

[103] Veneziano G., «Construction of a crossing-symmetric, Regge-behaved amplitude for linearly rising trajectories». Nuovo Cimento, 57 A, (1968), P. 190-197. 
[104] I. V. Volovich, «p-Adic string», Classical Quantum Gravity, Vol. 4, (1987), P. 83-87.

[105] Kushner A., Lychagin V., Rubtsov V., Contact Geometry and Nonlinear Differential equations., Cambridge University Press, (2007), 496 p.

[106] G. M. Zaslavsky, Chaos in Dynamic Systems, New-York: Harwood Academic Publishers, (1985), 370 p.

[107] P. Candelas, Gary T. Horowitz, Andrew Strominger, Edward Witten. Vacuum configurations for superstrings., Nuclear Physics B, Vol. 258, (1985), P. 46-74.

[108] W. Heisenberg «Über die Spektra von Atomsystemen mit zwei Elektronen». Z. Phys. 39, (1926), P. 499-518.

[109] P. W. Anderson, Theory of Magnetic Exchange Interactions, Exchange in Insulators and Semiconductors in Solid States Physics, Vol. 14, F. Seitz and D. Turnbull (eds.) Academic Press, New York, 1963.

[110] L. Landau, R. Pierts, Quantum Electrodynamics in Configuration Space, Zeit. f. Phys., Vol. 62, (1930), P. 188-200.

[111] A. T. Gavrilin, On the probability amplitude of photon's position, Vestnik of Lobachevsky State University of Nizhni Novgorod, N. 6(1), (2011), P. 70-74.

[112] S. L. McCall and E. L. Hahn, Self-induced Transparency by Pulsed Coherent Light, Phys. Rev. Lett., Vol. 18, (1967), P. 908-911.

[113] C. N. Yang, R. Mills. Conservation of Isotopic Spin and Isotopic Gauge Invariance, Phys. Rev., Vol. 96, N. 1, (1954), P. 191 - 195.

[114] Schwinger J. A Theory of the Fundamental Interactions, Ann. of Phys., Vol. 2, (1957), P. 407-434.

[115] J. Leite - Lopes, A model of the universal fermi interaction, Nucl. Phys., Vol. 8, (1958), P. 234-236.

[116] Salam A., Ward J. C., Weak and Electromagnetic Interactions, Nuovo Cimento, Vol. 11, (1959), P. 568-577.

[117] Sheldon L. Glashow, Partial-symmetries of weak interactions, Nucl. Phys., Vol. 22, (1961), P. 579-588.

[118] Salam A., Ward J. C., Electromagnetic and weak interactions, Phys. Lett., Vol. 13, (1964), P. 168-171.

[119] Faddeev L. D., Popov V. N. Feynman Diagrams for the Yang-Mills Field, Phys. Lett. B, Vol. 25, (1967), P. 29 - 30.

[120] Weinberg S., A model of leptons, Phys. Rev. Lett., Vol. 19, (1967), P. 1264-1266.

[121] Salam A., Week and Electromagnetic Interaction, Elementary Particle Theory, Ed. N. Svartholm. - Almguist and Wiksell, Stockh., (1968), P. 367-377. 
[122] G. 't Hooft, Renormalization of massless Yang-Mills fields, Nucl. Phys., Vol. B33, (1971), P. 173-199.

[123] G. 't Hooft, Renormalizable Lagrangians for massive Yang-Mills fields, Nucl. Phys., V. B35, (1971), P. 167-188.

[124] G. 't Hooft, M. Veltman. Regularization and Renormalization of Gauge Fields, Nuclear Physics B, Vol. 44, (1972), P. 189-219.

[125] L. D. Faddeev, Gauge Invariant Model of Electromagnetic and Week Interactions of Leptons, Report of Ac. of Sc. USSR, Vol. 210, N. 4, (1973), P. 807- 810.

[126] Arnison G. et al. (UA1), Experimental observation of isolated large transverse energy electrons with associated missing energy at $s=540 \mathrm{GeV}$, Phys. Lett. B, Vol. 122, (1983), P. 103-116.

[127] Banner M. et al. (UA2), Observation of single isolated electrons of high transverse momentum in events with missing transverse energy at the CERN pp collider, Phys. Lett. B, Vol. 122, (1983), P. 476-485.

[128] Arnison G. et al. (UA1), Experimental observation of lepton pairs of invariant mass around $95 \mathrm{GeV} / \mathrm{c} 2$ at the CERN SPS collider, Phys. Lett. B, Vol. 126, (1983), P. 398-410.

[129] Bagnaia P. et al. (UA2), Evidence for Z0 ---> e+ e- at the CERN anti-p p Collider, Phys. Lett. B, Vol. 129, (1983), P. 130-140.

[130] Arnison G. et al. (UA1), Further evidence for charged intermediate vector bosons at the SPS collider, Phys. Lett. B, Vol. 129, (1983), P. 273-282.

[131] Bagnaia P. et al. (UA2), A Study of High Transverse Momentum Electrons Produced in anti-p p Collisions at 540-GeV, Z. Phys. C (1), Vol. 24, (1984), P. 1-17.

[132] Andersen G. B. et al. Trapped antihydrogen. Nature 468, (2010), P. 673-676.

[133] Andersen G. B. et al. Confinement of antihydrogen for 1,000 seconds. Nat. Phys. 7, (2011), P. 558-564.

[134] The ALPHA Collaboration \& Charman A. E. Description and first application of a new technique to measure the gravitational mass of antihydrogen. Nat. Commun., Vol. 4, (2013), 1785.

[135] Pérez, P. et al. The GBAR antimatter gravity experiment. Hyperf. Interact., Vol. 233, (2015), P. $21-27$.

[136] Bertsche, W. A. Prospects for comparisons of matter and antimatter gravitation with ALPHA-g., Philosophical Transactions of The Royal Society A., (2018), 376 (2116): 20170265.

[137] Kostelecký V. A., Vargas A. J. Lorentz and CPT tests with hydrogen, antihydrogen, and related systems. Phys. Rev. D, Vol. 92, 056002, (2015).

[138] Ulmer, S. et al. Hight-precision comparison of the antiproton-to-proton charge-tomass ratio. Nature, Vol. 524, (2015), P. 196 - 199. 
[139] Crivelli P., Cooke D., Heiss M. W. Antiproton charge radius. Phys. Rev. D, Vol. 94, (2016), 052008.

[140] Hori, M. et al. Buffer-gas cooling of antiprotonic helium to 1.5 to $1.7 \mathrm{~K}$, and antiproton-to-electron mass ratio. Science, Vol. 354, (2016), P. 610 - 614.

[141] Ahmadi, M. et al. Observation of the 1S-2S transition in trapped antihydrogen. Nature, Vol. 541, (2017), P. 506 - 510.

[142] Ahmadi, M. et al. Observation of the hyperfine spectrum of antihydrogen. Nature, Vol. 548, (2017), P. 66 - 69.

[143] Ahmadi, M. et al. Antihydrogen accumulation for fundamental symmetry tests. Nat. Commun., Vol. 8, (2017), P. 681.

[144] Smorra, C. A parts-per-billion measurement of the antiproton magnetic moment. Nature, Vol. 550, (2017), P. 371 - 374.

[145] Rasmussen C. 0., Madsen N. \& Robicheaux F. Aspects of 1S-2S spectroscopy of trapped antihydrogen atoms. J. Phys. B, Vol. 50, 184002, (2017); corrigendum Vol. 51, 099501, (2018).

[146] Ahmadi, M. et al. Characterisation of the 1S-2S transition in antihydrogen. Nature, Vol. 557, (2018), P. $71-75$.

[147] Ahmadi, M. et al. Observation of the 1S-2P Lyman- $\alpha$ transition in antihydrogen. Nature, Vol. 561, (2018), P. 211-215.

[148] Erikson S. Precision measurements on trapped antihydrogen in the ALPHA experiment. Philos. Trans. Royal Soc. A, Vol. 376, (2018), 20170268.

[149] Capra A. \& ALPHA Collaboration. Lifetime of magnetically trapped antihydrogen in ALPHA. Hyperfine Interact. 240, 9 (2019).

[150] S. Sala, A. Ariga, A. Ereditato, R. Ferragut, M. Giammarchi, M. Leone, C. Pistillo, P. Scampoli «First demonstration of antimatter wave interferometry», Science Advances, Vol. 5, No. 5, eaav7610, (2019).

[151] The ALPHA Collaboration. Investigation of the fine structure of antihydrogen. Nature 578, (2020), P. 375-380.

[152] R. P. Feynman and M. R. Hibbs, Quantum mechanics and path integrals, (McGraw Hill, New York, 1965), 365 p.

[153] Handjian Ling, Guillam E. Mclvor, Kasper van der Vaart, Richard T. Vaughan, Alex Thornton, Nicholas T. Ouellette, Costs and Benefits of Social Relationships in the Collective Motion of Bird Flocks, Nature Ecology \& Evolution, 3, (2019), P. 943-948.

[154] Nicolas Manton and Paul Sutcliffe, Topological Solitons. Cambridge University Press, N.-Y., (2004), 506 p. 
[155] V. E. Zakharov, A. B. Shabat, Exact Theory of Two-dimensional Self-focusing and Onedimensional Self-modulation of Waves in Nonlinear Media, JETP Letter, Vol. 61, N. 1, (1971), P. 118-134.

[156] V. E. Zakharov, L. A. Takhtadzhyan, L.D. Faddeev, Complete description of solutions of the 'sine-Gordon' equation, Sov. Phys. Dokl., Vol. 219, N. 6, (1974), P. 1334-1337.

[157] Zakharov V. E. (ed.) “What is integrability?”, Springer-Verlag, (1992), XIV+321 p.

[158] V. E. Zakharov, E. A. Kuznetsov, Solitons and collapses: two evolution scenarios of nonlinear wave systems, UFN, Vol. 182, (2012), P. 569 - 592.

[159] O. M. Braun, Y. S. Kivshar, The Frenkel-Kontorova Model. Concept, Methods and Applications. SPRINGER, (2004), 536 p.

[160] A. S. Fokas «Integrable Nonlinear Evolution Partial Differential Equation in $4+2$ and 3+1 Dimensions», Phys. Rev. Lett., Vol. 96, (2006), 190201. 


\section{Благодарность}

Посвящаю эту работу - моим родителям: отцу Авшалумову Шамаилу Шумуновичу (1937-2012), $\mathrm{PhD}$ технические науки (1983) и матери Авшалумовой Мальке Юсуфовне (1941 г.р.), химику. 